\title{
ELASTOHYDRODYNAMIC ANALYSIS OF A ROTARY LIP SEAL USING FLOW FACTORS
}

\author{
A Thesis \\ Presented to \\ The Academic Faculty \\ by
}

Ann H. Rocke

\author{
In Partial Fulfillment \\ of the Requirements for the Degree \\ Master of Science in Mechanical Engineering
}

Georgia Institute of Technology

July 2004 


\section{ELASTOHYDRODYNAMIC ANALYSIS OF A ROTARY LIP SEAL USING FLOW FACTORS}

Approved:

Advisor, Richard F. Salant

Jeffrey L. Streator

Steven Danyluk

July 30,2004 


\section{ACKNOWLEDGEMENTS}

I would like to thank my advisor, Dr. Richard Salant for the guidance, encouragement and support he provided throughout my graduate studies. It was an honor to know him and to have benefited from the wealth of his knowledge and experience. I would also like to thank my committee members, Dr. Jeffrey Streator and Dr. Steven Danyluk for their suggestions and expertise.

I am grateful to Rockwell Automation and Georgia Power Co. for providing the financial support for this work. 


\section{TABLE OF CONTENTS}

ACKNOWLEDGEMENTS

LIST OF TABLES vi vi

LIST OF FIGURES vii

NOMENCLATURE $\quad$ X

SUMMARY X Xiii

CHAPTER I INTRODUCTION

CHAPTER II BACKGROUND

2.1 Average Flow Model with Three-Dimensional Roughness 5

2.2 Cavitating Flow between Rough Surfaces 5

2.3 The Effects of Roughness Orientation 6

CHAPTER III PROBLEM FORMULATION 7

$\begin{array}{ll}3.1 \text { Configuration } & 7\end{array}$

3.2 Fluid Mechanics Analysis $\quad 8$

3.2.1 Flow Factors

3.2.2 Interpolation and Extrapolation of Shear Flow Factors $\quad 14$

$\begin{array}{ll}\text { 3.3 Deformation Analysis } & 20\end{array}$

3.4 Finite Element Analysis 22

3.5 Asperity Distortion Analysis 26 
CHAPTER IV NUMERICAL SCHEME

4.1 Discretization of Reynolds Equation

4.2 Computational Procedure

CHAPTER V RESULTS

5.1 Preliminary Hydrodynamic Analysis

5.2 Implementing the Normal Deformation Calculation 


\section{LIST OF TABLES}

Table 3.1 Coefficients of Equations 3.12, 3.13 (Harp and Salant, 2001) 11

Table 3.2 Coefficients of Equation 3.16 (Harp and Salant, 2001) 11

Table 3.3 Coefficients of Equation 3.17 (Harp and Salant, 2001) 11

Table 3.4 Coefficients of Equation 3.15 (Harp and Salant, 2001) 12

Table 3.5 Coefficients of Equation $3.19 \quad 16$

Table 3.6 Coefficients of Equation 3.20 16

Table 3.7 Coefficients of Equation 3.21 17

$\begin{array}{lll}\text { Table } 3.8 & \text { Coefficients of Equation } 3.25 & 18\end{array}$

Table 3.9 Coefficients of Equation $3.29 \quad 19$ 


\section{LIST OF FIGURES}

Figure 1.1 Sealing zone 2

Figure 3.1 Boundary Conditions and Asperity Orientation Pattern 8

Figure 3.2 Shear Flow Factors in Distinct Form and Unified Curve Fit (Harp and 11

Salant, 2001)

Figure 3.3 Various Asperity Orientations as Defined by $\gamma$

Figure 3.4 Interpolated Shear Flow Factor Values between $\mathrm{H}=3$ and 4 for $\gamma=$ $1 / 9$, Showing Points of Intersection with the Unified Curve

Figure 3.5 Distorted Asperity Pattern

Figure 3.6 Detailed Cross-Section of Lip Seal Rubber (millimeters)

Figure 3.7 Deformed Results from Contact Model

Figure 3.8 Contact Pressure Distribution before Breakage

Figure 3.9 Influence Coefficients for Normal Deformation 25

Figure 3.10 Influence Coefficients for Shear Deformation 26

Figure 3.11 Asperity Distortion as Simulated with the Rotation of Axes 27

Figure 3.12 Transformation using Rotation of Axes (b) and $\mathrm{X}+\delta(\mathrm{Y}), \mathrm{Y}(\mathrm{c})$

Figure 4.1 Diagram of the Control Volume for the Discretization Process 33

Figure 4.2 Computational Scheme 40

Figure 5.1 Pressure Distribution, Base Case, $\hat{y}_{b}=0.7$

Figure 5.2 Pressure Distribution, $\hat{y}_{b}=0.3$

$\begin{array}{lll}\text { Figure 5.3 Load Support vs. Speed } & 44\end{array}$

Figure 5.4 Pumping Rate vs. Speed 45

Figure 5.5 Load Support vs. Average Film Thickness 46 
Figure 5.6 Pumping Rate vs. Average Film Thickness 46

Figure 5.7 Load Support vs. Elasticity Parameter 47

Figure 5.8 Pumping Rate vs. Elasticity Parameter $\quad 48$

Figure 5.9 Load Support vs. $\hat{y}_{b} \quad 49$

Figure 5.10 Pumping Rate vs. $\hat{y}_{b} \quad 49$

Figure 5.11 Load Support vs. Sealed Pressure $\quad 50$

Figure 5.12 Pumping Rate vs. Sealed Pressure $\quad 50$

Figure 5.13 Calculated Average Film Thickness Distribution 52

Figure 5.14 Pressure Distribution for $H=2.00$ before Calculation of Normal 53 Deformation

Figure 5.15 Pressure distribution for $\mathrm{H}=1.74$ using Extrapolation \& Normal 53

Deformation Analysis

Figure 5.16 Pumping Rate vs. Average Film Thickness 54

Figure 5.17 Load Support vs. Average Film Thickness 54

Figure 5.18 Contact Pressure and Fluid Pressure Distributions 56

Figure 5.19 The Effect of Shaft Speed on Avg. Film Thickness 57

Figure 5.20 The Effect of Shaft Speed on Shear Deformation 57

Figure 5.21 Pumping Rate vs. Speed 58

Figure 5.22 Load Support vs. Speed 58

Figure 5.23 Power Loss vs. Speed 59

Figure 5.24 Average Film Thickness vs. Speed 60

Figure 5.25 Maximum Shear Deformation vs. Speed 60

Figure 5.26 Pumping Rate vs. Viscosity 61

$\begin{array}{lll}\text { Figure 5.27 Load Support vs. Viscosity } & 62\end{array}$ 
$\begin{array}{lll}\text { Figure 5.28 } & \text { Power Loss vs. Viscosity } & 62\end{array}$

Figure 5.29 Average Film Thickness vs. Viscosity 63

Figure 5.30 Maximum Shear Deformation vs. Viscosity 64

$\begin{array}{lll}\text { Figure 5.31 Pumping Rate vs. RMS Roughness } & 65\end{array}$

Figure 5.32 Dimensional Pumping Rate vs. RMS Roughness 65

Figure 5.33 Load Support vs. RMS Roughness 65

Figure 5.34 Power Loss vs. RMS Roughness 66

Figure 5.35 Dimensional Power Loss vs. RMS Roughness 66

Figure 5.36 Average Film Thickness vs. RMS Roughness 67

Figure 5.37 Maximum Shear Deformation vs. RMS Roughness 67

$\begin{array}{lll}\text { Figure 5.38 Lift-Off Speed vs. Viscosity } & 68\end{array}$

Figure 5.39 Dimensional Lift-Off Speed vs. Viscosity 68

Figure 5.40 Lift-Off Speed vs. RMS Roughness 69

Figure 5.41 Dimensional Lift-Off Speed vs. RMS Roughness 69

Figure 5.42 Pumping Rate vs. Asperity Aspect Ratio 70

Figure 5.43 Load Support vs. Asperity Aspect Ratio 70

Figure 5.44 Power Loss vs. Asperity Aspect Ratio 71

Figure 5.45 Average Film Thickness vs. Asperity Aspect Ratio 71

Figure 5.46 Maximum Shear Deformation vs. Asperity Aspect Ratio 72 


\section{NOMENCLATURE}

$\hat{A}_{1}$

E

$H$

$I_{1}$

$I_{2}$

$L_{x} \quad$ length of solution domain in x-direction

$L_{y} \quad$ length of solution domain in y-direction

$P$

$p_{a}$

$p_{c}$

$p_{s}$

$\hat{p}_{s}$

$\hat{p}_{\text {contact }}$

$\hat{p}_{d e f}$

$\hat{Q}_{y}$

$\hat{q}_{x}$

$\hat{q}_{y}$

$\hat{W} \quad$ dimensionless load support, $\mathrm{W} /\left(\mathrm{p}_{\mathrm{a}}-\mathrm{p}_{\mathrm{c}}\right) \mathrm{L}_{\mathrm{x}} \mathrm{L}_{\mathrm{y}}$

$\hat{R}$

$U$

elastic modulus

deformation

deformation

dimensionless pressure, $\left(\mathrm{p}-\mathrm{p}_{\mathrm{c}}\right) /\left(\mathrm{p}_{\mathrm{a}}-\mathrm{p}_{\mathrm{c}}\right)$

ambient pressure

cavitation pressure (set equal to zero)

sealed pressure

$\mathrm{p}_{\text {contact }} / \mathrm{E}$

$\left.\mathrm{p}_{\mathrm{c}}\right) / \mathrm{E}+\mathrm{p}_{\mathrm{c}} / \mathrm{E}$ y-direction $12 \mu L_{x} q_{x} /\left[\left(p_{a}-p_{c}\right) \sigma^{3}\right]$ $\mathrm{x}$-direction $12 \mu \mathrm{L}_{\mathrm{x}} \mathrm{q}_{\mathrm{y}} /\left[\left(\mathrm{p}_{\mathrm{a}}-\mathrm{p}_{\mathrm{c}}\right) \sigma^{3}\right]$

dimensionless seal radius, $R / L_{y}$

surface speed of shaft dimensionless static undeformed film thickness, $A_{1} / \sigma$

dimensionless average film thickness in $\mathrm{x}$-direction, $\mathrm{h} / \sigma$

dimensionless influence coefficient for normal (radial)

dimensionless influence coefficient for shear (circumferential)

dimensionless sealed pressure, $\left(\mathrm{p}_{\mathrm{s}}-\mathrm{p}_{\mathrm{c}}\right) /\left(\mathrm{p}_{\mathrm{a}}-\mathrm{p}_{\mathrm{c}}\right)$

dimensionless contact pressure for deformation analysis,

dimensionless pressure for deformation analysis, $\mathrm{P}\left(\mathrm{p}_{\mathrm{a}}{ }^{-}\right.$

dimensionless pumping rate in y-direction, $12 \mu \mathrm{Q}_{\mathrm{y}} /\left[\left(\mathrm{p}_{\mathrm{a}}-\mathrm{p}_{\mathrm{c}}\right) \sigma^{3}\right]$

dimensionless flow rate in the $\mathrm{x}$-direction per unit length in the

dimensionless flow rate in the y-direction per unit length in the non-dim

$\mathrm{Pa}$

non-dim

non-dim

non-dim

M

M

non-dim

$\mathrm{Pa}$

$\mathrm{Pa}$

$\mathrm{Pa}$

non-dim

non-dim

non-dim

non-dim

non-dim

non-dim

non-dim

non-dim

$\mathrm{m} / \mathrm{s}$ 


\begin{tabular}{|c|c|c|}
\hline$V_{r}$ & $\begin{array}{l}\text { dimensionless variance ratio, }\left(\sigma_{1} / \sigma\right)^{2}\left(V_{r}=1 \text { for case with one }\right. \\
\text { smooth surface })\end{array}$ & non-dim \\
\hline$X$ & local $\mathrm{x}$-coordinate used in asperity distortion analysis & $\mathrm{m}$ \\
\hline$\hat{x}$ & dimensionless circumferential coordinate, $\mathrm{x} / \mathrm{L}_{\mathrm{x}}$ & non-dim \\
\hline Y & local y-coordinate used in asperity distortion analysis & $\mathrm{m}$ \\
\hline$\hat{y}$ & dimensionless axial coordinate, $y / \mathrm{L}_{\mathrm{y}}$ & non-dim \\
\hline$y_{b}$ & $\begin{array}{l}\text { y-location of maximum circumferential lip surface } \\
\text { displacement }\end{array}$ & $\mathrm{m}$ \\
\hline$\hat{y}_{b}$ & $\begin{array}{l}\text { dimensionless y-location of maximum circumferential lip } \\
\text { surface displacement, } \mathrm{y}_{\mathrm{b}} / \mathrm{L}_{\mathrm{y}}\end{array}$ & non-dim \\
\hline $\mathrm{z}$ & dimensionless statistical surface height, $\mathrm{H} / 3$ & non-dim \\
\hline$\alpha$ & dimensionless parameter describing elasticity of lip & non-dim \\
\hline$\hat{\delta}$ & dimensionless circumferential displacement of lip surface, $\delta / L_{x}$ & non-dim \\
\hline $\begin{array}{l}\phi_{f}, \phi_{f s s} \\
\phi_{f p p}, \Phi_{f s}\end{array}$ & dimensionless shear stress factors & non-dim \\
\hline$\phi_{c . c}$ & dimensionless density flow factor & non-dim \\
\hline$\phi_{s . c . x}, \phi_{s . c . y}$ & dimensionless shear flow factors & non-dim \\
\hline $\begin{array}{l}\phi_{x x}, \phi_{x y} \\
\phi_{y x}, \phi_{y y}\end{array}$ & dimensionless pressure flow factors & non-dim \\
\hline$\gamma$ & dimensionless aspect ratio of asperity, $\lambda_{\mathrm{x}} \lambda_{\mathrm{y}}$ with $\theta=0$ & non-dim \\
\hline K & dimensionless (aspect ratio of solution space) ${ }^{2}, \mathrm{~L}_{\mathrm{x}}{ }^{2} / \mathrm{L}_{\mathrm{y}}{ }^{2}$ & non-dim \\
\hline$\lambda_{x}$ & autocorrelation length in $\mathrm{x}$-direction & $\mathrm{m}$ \\
\hline$\lambda_{y}$ & autocorrelation length in y-direction & $\mathrm{m}$ \\
\hline$\mu$ & viscosity & $\mathrm{Pa}-\mathrm{s}$ \\
\hline$\hat{\mu}$ & dimensionless viscosity $\left(\mu / \mu_{\text {ref }}\right)$ & non-dim \\
\hline$\hat{\Pi}$ & dimensionless power consumption, $\Pi \mu /\left[\mathrm{E}(2 \pi) \sigma^{2}\left(\mathrm{p}_{\mathrm{a}}-\mathrm{p}_{\mathrm{c}}\right) \mathrm{L}_{\mathrm{x}}\right]$ & non-dim \\
\hline$\theta$ & orientation angle of asperities & radians \\
\hline$\sigma$ & rms roughness of lip surface & $\mathrm{m}$ \\
\hline
\end{tabular}




$\begin{array}{llr}\hat{\sigma} & \text { dimensionless rms roughness of lip surface, } \sigma / \sigma_{\text {ref }} & \text { non-dim } \\ \hat{\tau}_{\text {avg }} & \text { average dimensionless shear stress in the x-direction, } \tau_{\mathrm{avg}} / \mathrm{E} & \text { non-dim } \\ \Psi & \text { dimensionless cavitation parameter, }\left(\mu|\mathrm{U}| \lambda_{\mathrm{x}}\right) /\left(\bar{p}-p_{c}\right) \sigma^{2} & \text { non-dim } \\ \zeta & \text { dimensionless shaft speed, }\left(\mu \mathrm{UL}_{\mathrm{x}}\right) /\left[\left(\mathrm{p}_{\mathrm{a}}-\mathrm{p}_{\mathrm{c}}\right) \sigma^{2}\right] & \text { non-dim }\end{array}$

superscripts

$\wedge \quad$ dimensionless quantity

mean value 


\section{SUMMARY}

An elastohydrodynamic analysis of a rotary lip seal is performed numerically, incorporating both the fluid mechanics of the lubricating film and the elastic deformation of the lip, by solving the Reynolds equation with flow factors. Asperities on the lip surface dominate the behavior of the flow field in the lubricating film and the elastic deformation of the lip. Since previous analyses treated those asperities deterministically, they required very large computation times. The present approach is much less computationally intensive because the asperities are treated statistically.

Since cavitation and asperity orientation play important roles, these are taken into account in the computation of the flow factors. An asperity distortion analysis is introduced to obtain a more realistic model of the complex variations in the asperity distribution on the surface of the seal.

Results of the analysis show how the operating parameters of the seal and the characteristics of the asperities affect such seal characteristics as the thickness of the lubricating film, reverse pumping rate, power dissipation and load carrying capacity. 


\section{CHAPTER I \\ INTRODUCTION}

To a casual observer, the manner in which a rotary lip seal retains liquid appears to be self-evident. The interference fit between the rotating shaft and the lip, combined with the tension of the garter spring seems to form a flexible yet impenetrable impasse between liquid and air. Upon closer inspection, however, questions regarding the sealing mechanism become apparent. Is a lubricating film required at the interface to maintain the integrity of the lip and prevent excessive wear, and if so, why isn't the oil leaking out?

The rotary lip seal is the type of seal most widely used in industry today for rotating shaft applications (Horve, 1996). For the better part of a century it has been analyzed largely by means of expensive and time consuming experimental testing. The complex nature of its sealing mechanism has not been fully understood until recent years.

In a previous study by Salant (1999), it was shown that the lip seal operates under normal conditions with a continuous lubricating film under the lip as depicted in Figure 1.1. In a successful seal, the microgeometry of the lip surface in the sealing zone makes two important contributions. First, the asperities under the lip act as mini slider bearings, producing local pressure elevations as the rotating shaft pulls the fluid along, in between itself and the rubber. This provides load support that keeps the lip lifted off of the seal. Secondly, as shown by Kammüller (1986) and Müller (1987), the asperity pattern deforms in such a way that it acts as a shear pump, inducing a reverse pumping action that pumps the fluid away from the air-side. This is called reverse pumping because it 
opposes the expected leakage flow that is being driven by the higher pressure on the liquid-side.

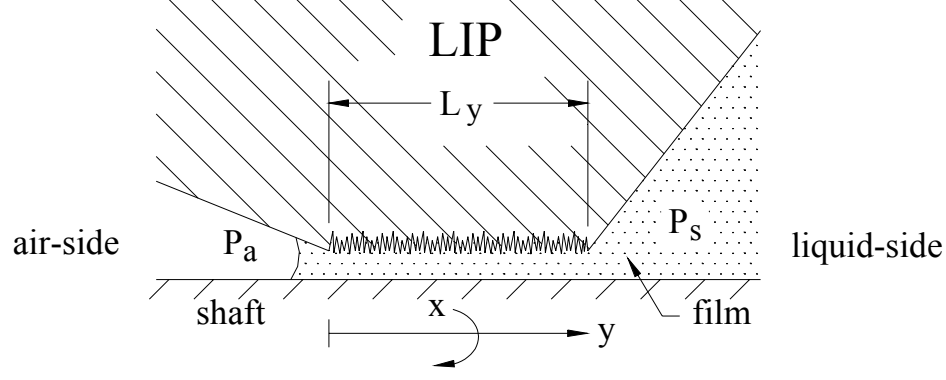

Figure 1.1 Sealing zone

In order to conduct a thorough analysis that encompasses the complex behaviors of a successfully functioning lip seal, the construction of an elastohydrodynamic model is necessary. Such a model consists of a hydrodynamic analysis of the flow in the lubricating film, coupled with a deformation analysis of the lip material. The hydrodynamic analysis provides solutions for the pressure and shear stress distributions, which determine the deformation of the rubber. The deformation analysis produces the film thickness distribution which affects the hydrodynamic results. An iterative computation procedure is required to solve this combination of dependent effects. The procedure consists of a nested loop configuration in which the hydrodynamic analysis is conducted in an inner iteration loop, and then an outer loop performs the deformation analysis. For the hydrodynamic analysis, a numerical scheme is used to solve the Reynolds equation with flow factors. The deformation analysis is performed using data that is obtained from a finite element analysis of a lip seal.

In previous elastohydrodynamic studies (Salant and Flaherty, 1994, 1995; Salant, 1996; Shi and Salant, 2000; Shi and Salant, 2001; Shen and Salant, 2003) the lip seal has 
been analyzed deterministically, requiring the governing equations to be solved at every point in the sealing zone. These models have produced a wealth of information and understanding about the physical mechanisms that govern lip seal behavior. Their findings have confirmed data from previous conceptual models and corresponded qualitatively with past experimental results; however, the methodology requires a very large computation time. Thus, while these deterministic models provide successful analyses of lip seal behavior, they are limited in their use as a design tool.

In this work, a statistical approach is used that greatly reduces the required computation time. With this approach, it is not necessary to solve the governing equations for the flow field around each asperity. Instead, the flow factor method (Patir and Cheng, 1978; Patir and Cheng, 1979) is used, which allows the Reynolds equation to be defined in terms of the average surface roughness on a macroscopic scale based on statistical data of the microscopic roughness. This method, enhanced by the extended flow factor work of Harp and Salant (2001) provides the means for a more efficient study of lip seal performance.

The original and modified flow factor methods mentioned above are limited in that they only consider asperities with the major axis parallel or normal to the direction of flow. However, it is important to model the lip's continuously varying asperity pattern as it is crucial to successful lip seal behavior. This is made possible by the development of a method that transforms flow factors for parallel and normal asperity orientation to that of any arbitrary asperity orientation (Lo, 1992).

As in the previous elastohydrodynamic studies in which the lip seal is analyzed deterministically, this approach consists of a fluid mechanics analysis of the flow field in 
the lubricating film and a structural analysis of the lip deformations. Additionally, an asperity distortion analysis is developed that calculates the change in surface characteristics when the surface is circumferentially displaced.

A description of the background required to perform this work is provided in the following chapter. Chapters three and four explain the configuration for and formulation of the model for this elastohydrodynamic analysis. In the final chapter, results for different phases of the model are revealed and discussed as they apply to the application of a rotary lip seal. 


\section{CHAPTER II}

\section{BACKGROUND}

\subsection{Average Flow Model with Three-Dimensional Roughness}

A new approach was developed by Patir and Cheng $(1978,1979)$ for the study of flow over isotropic or non-isotropic three-dimensional surface roughness. They recognized the limitations of the stochastic average film thickness models (Tzeng and Saibel, 1967) that were currently in use and introduced a new method of deriving an average Reynolds equation that includes pressure and shear flow factors. The flow factors are functions of surface characteristics that are obtained through flow simulations conducted using randomly generated or measured surface roughness. This method can be used to analyze any type of roughness structure and can be extended to the partial lubrication regime $(\mathrm{h} / \sigma<3)$ where the effect of roughness is critical. This average flow model greatly improved computational efficiency; however it does not account for cavitation which is known to exist in the sealing zone of a rotary lip seal (Stakenborg, 1988) and plays an important role in lip seal behavior.

\subsection{Cavitating Flow between Rough Surfaces}

Harp and Salant (2001) recognized the need for a general flow model that numerically analyzes inter-asperity cavitation, a phenomenon that must be considered in order to accurately predict lubricant performance. Cavitation is generated when a diverging surface moves relative to another and air, dissolved in oil, comes out of solution. A diverging surface causes a drop in fluid pressure and the lubricant will 
cavitate if the cavitation pressure is reached. In the sealing zone of a lip seal it is the microscopic topography of the surface, specifically the valleys between the asperities, which provide the regions of divergent film that cause cavitation. The general model that was developed to address this consists of an average Reynolds equation, modified with flow factors that simulate the combined effects of surface roughness and inter-asperity cavitation.

\subsection{The Effects of Roughness Orientation}

The studies described above are limited to asperities with the major axis parallel or perpendicular to the direction of motion. In the lip seal, the asperity orientation varies continuously over the sealing zone, and that varying pattern is crucial to the load carrying capacity and pumping mechanisms for a successful seal. A method of transforming the parallel and perpendicular flow factors to those for any arbitrary orientation was developed by Lo (1992). The method is capable of dealing with the change in roughness orientation and introduces a modified Reynolds equation with flow factors that are functions of an initial parallel or perpendicular configuration that has been transformed to an orientation angle, $\theta$. 


\section{CHAPTER III \\ PROBLEM FORMULATION}

\subsection{Configuration}

The configuration for the analysis is contained in Figure 3.1a, which shows two surfaces separated by a fluid film. The upper surface represents the seal lip, while the lower surface represents the shaft. The $\mathrm{x}$-direction represents the circumferential direction, while the $y$-direction respresents the axial direction (see Figure 1.1). $\mathrm{L}_{\mathrm{y}}$ is the axial width of the sealing zone, while $\mathrm{L}_{\mathrm{x}}$ is chosen to be of the same order as $\mathrm{L}_{\mathrm{y}}$. Thus, the analysis considers a small section of the sealing zone that spans its axial width. The same pressure is applied on both of the y boundaries while cyclic conditions are applied on the $\mathrm{x}$ boundaries. The upper surface is rough and stationary and the bottom surface is smooth and moving at a non-zero velocity in the $\mathrm{x}$-direction. Figure $3.1 \mathrm{~b}$ shows an approximation of the known asperity orientation for an operating lip seal consisting of an arc shaped formation that reaches its peak at a point about $70 \%$ of the distance in the $y-$ direction and is repetitive in the $\mathrm{x}$-direction. This approximation is used in the preliminary hydrodynamic analysis. The dashed lines schematically illustrate the orientation of the major axes of the asperities that lie on the lip surface. 


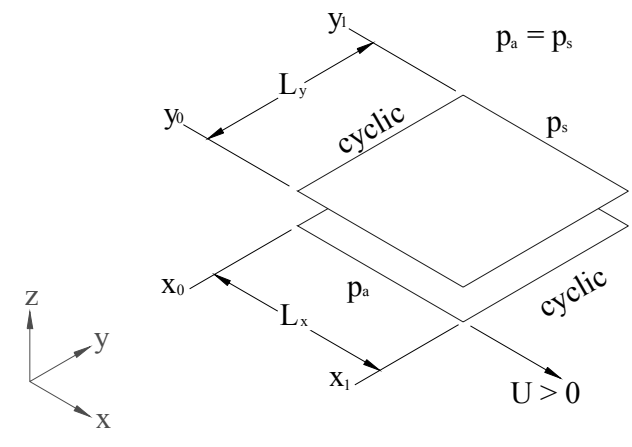

(a)

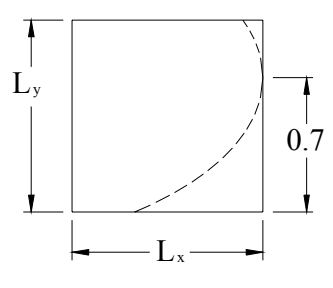

(b)

Figure 3.1 Boundary Conditions and Asperity Orientation Pattern

\subsection{Fluid Mechanics Analysis}

A modified Reynolds equation was derived by considering the flow in the $\mathrm{x}$ and $\mathrm{y}$ directions, with sliding only in the x direction (Harp and Salant, 2001; Salant and Rocke, 2004), resulting in the following flow rates for the flow factor method,

$$
\begin{aligned}
& q_{x}=-\frac{h^{3}}{12 \mu}\left(\phi_{x x} \frac{\partial p}{\partial x}+\phi_{x y} \frac{\partial p}{\partial y}\right)+\frac{U}{2}\left(h-\sigma \phi_{c . c}\right)+\frac{U}{2} \sigma \phi_{s . c . x} \\
& q_{y}=-\frac{h^{3}}{12 \mu}\left(\phi_{y x} \frac{\partial p}{\partial x}+\phi_{y y} \frac{\partial p}{\partial y}\right)+\frac{U}{2} \sigma \phi_{\text {s.c. }}
\end{aligned}
$$

The first term on the right hand side of Equation 3.1 is the flow in the $\mathrm{x}$-direction due to the pressure gradients in the $\mathrm{x}$ - and $\mathrm{y}$-directions, and the remaining terms are the flow in the $\mathrm{x}$-direction due to the motion of the shaft. The first term on the right hand side of Equation 3.2 is the flow in the $\mathrm{y}$-direction due to the pressure gradients in the $\mathrm{x}$ - 
and y-directions, and the remaining term is the flow in the y-direction due to the motion of the shaft. $\phi_{\mathrm{xx}}, \phi_{\mathrm{xy}}, \phi_{\mathrm{yy}}$ and $\phi_{\mathrm{yx}}$ are the pressure flow factors, $\phi_{\mathrm{s}, \mathrm{c}, \mathrm{x}}$ and $\phi_{\mathrm{s}, \mathrm{c}, \mathrm{y}}$ are the shear flow factors, and $\phi_{c, c}$ is the density flow factor.

Substituting Equations 3.1 and 3.2 into the steady flow continuity equation,

$\frac{\partial q_{x}}{\partial x}+\frac{\partial q_{y}}{\partial y}=0$

gives,

$$
\begin{aligned}
& \frac{\partial}{\partial x}\left[\frac{h^{3}}{12 \mu}\left(\phi_{x x} \frac{\partial p}{\partial x}+\phi_{x y} \frac{\partial p}{\partial y}\right)\right]+\frac{\partial}{\partial y}\left[\frac{h^{3}}{12 \mu}\left(\phi_{y x} \frac{\partial p}{\partial x}+\phi_{y y} \frac{\partial p}{\partial y}\right)\right] \\
& =\frac{U}{2} \frac{\partial}{\partial x}\left(h-\sigma \phi_{c . c}\right)+\frac{U}{2} \sigma\left(\frac{\partial \phi_{s . c . x}}{\partial x}+\frac{\partial \phi_{s . c . y}}{\partial y}\right)
\end{aligned}
$$

Introducing the dimensionless variables,

$$
\hat{x}=\frac{x}{L_{x}}, \quad \hat{y}=\frac{y}{L_{y}}, \quad H=\frac{h}{\sigma}, \quad \mathrm{K}=\frac{L_{x}}{L_{y}}, \quad P=\frac{p}{p_{s}-p_{c}}, \quad \zeta=\frac{\mu U L_{X}}{\left(p_{s}-p_{c}\right) \sigma^{2}}
$$

Equation 3.4 can be written as,

$$
\begin{aligned}
& \frac{\partial}{\partial \hat{x}}\left[H^{3}\left(\phi_{x x} \frac{\partial P}{\partial \hat{x}}+\mathrm{K} \phi_{x y} \frac{\partial P}{\partial \hat{y}}\right)\right]+\mathrm{K} \frac{\partial}{\partial \hat{y}}\left[H^{3}\left(\phi_{y x} \frac{\partial P}{\partial \hat{x}}+\mathrm{K} \phi_{y y} \frac{\partial P}{\partial \hat{y}}\right)\right] \\
& =6 \zeta \frac{\partial}{\partial \hat{x}}\left(H-\phi_{c . c}\right)+6 \zeta\left(\frac{\partial \phi_{s . c . x}}{\partial \hat{x}}+\mathrm{K} \frac{\partial \phi_{s . c . y}}{\partial \hat{y}}\right)
\end{aligned}
$$

\subsubsection{Flow Factors}

Lo (1992) developed a method capable of dealing with the change of surface roughness orientation that calculates the following "skewed" flow factors: 


$$
\begin{aligned}
& \phi_{x x}=\cos ^{2} \theta \cdot\left(\phi_{x}\right)_{\theta=0}+\sin ^{2} \theta \cdot\left(\phi_{x}\right)_{\theta=90} \\
& \phi_{y y}=\cos ^{2} \theta \cdot\left(\phi_{y}\right)_{\theta=0}+\sin ^{2} \theta \cdot\left(\phi_{y}\right)_{\theta=90} \\
& \phi_{x y}=\phi_{y x}=\sin \theta \cos \theta \cdot\left[\left(\phi_{x}\right)_{\theta=0}-\left(\phi_{x}\right)_{\theta=90}\right] \\
& \phi_{s . c . x}=\cos ^{2} \theta \cdot\left(\phi_{s . c}\right)_{\theta=0}+\sin ^{2} \theta \cdot\left(\phi_{s . c}\right)_{\theta=90} \\
& \phi_{s . c . y}=\sin \theta \cos \theta \cdot\left[\left(\phi_{s . c}\right)_{\theta=90}-\left(\phi_{s . c}\right)_{\theta=0}\right] \\
& \phi_{c . c}=\cos ^{2} \theta \cdot\left(\phi_{c}\right)_{\theta=0}+\sin ^{2} \theta \cdot\left(\phi_{c}\right)_{\theta=90}
\end{aligned}
$$

The skewed flow factors are calculated using the flow factors for $\theta=0$ and $90^{\circ}$, developed by Harp and Salant (2001), Figures 3.12 - 3.17, which assume Gaussian distributions of surface height with some interpolation. The pressure flow factors, $\phi_{\mathrm{xx}}$, $\phi_{\mathrm{xy}}, \phi_{\mathrm{yy}}$ and $\phi_{\mathrm{yx}}$ are functions of the average film thickness $\mathrm{H}$, the aspect ratio of the asperities $\gamma$, and the asperity orientation angle $\theta$. The density flow factor $\phi_{c, c}$ and the shear flow factors, $\phi_{\text {s.c,x }}$ and $\phi_{\text {s.c,y }}$ are dependent on the cavitation parameter, $\Psi$ as well as on $\mathrm{H}$ and $\gamma$ (Harp and Salant, 2001). The coefficients $\mathrm{c}_{1}, \mathrm{r}, \mathrm{m}, \mathrm{c}_{2}, \mathrm{a}_{1}, \mathrm{~b}_{1}, \mathrm{a}_{2}$ and $\mathrm{b}_{2}$ are given in Tables $3.1-3.4$.

$$
\begin{aligned}
& \phi_{x}(H, \gamma)=1-c_{1} \exp (-r H) \quad(\text { for } \gamma \leq 1) \\
& \phi_{x}(H, \gamma)=1+c_{1}(H)^{-\mathrm{r}} \quad(\text { for } \gamma>1) \\
& \phi_{y}(H, \gamma)=\phi_{x}\left(H, \frac{1}{\gamma}\right) \quad\left(\text { for } \Psi(H)^{-2}<\right.\text { limit in table 3.4) } \\
& \phi_{s . c}(H, \gamma, \Psi)=-m \Psi(H)^{-2}+\mathrm{c}_{2} \quad \\
& \phi_{s . c}(H, \gamma, \Psi)=\frac{1}{a_{1}+b_{1} \Psi(H)^{-2}} \quad\left(\text { for } \Psi(H)^{-2} \geq\right. \text { limit in table 3.4) } \\
& \phi_{c}(H, \gamma, \Psi)=\frac{1}{a_{2}+b_{2} \Psi^{-1.5}(H)^{6.0}}
\end{aligned}
$$


When plotted against $\Psi(\mathrm{H})^{-2}$, the shear flow factors for each film thickness remain distinct, Equation 1.15, up to unique points at which they join a unified curve, Equation 1.16. An example of this for $\gamma=1$ and coefficients from Table 3.4 for specific values of $\mathrm{H}$ is shown in Figure 3.2.

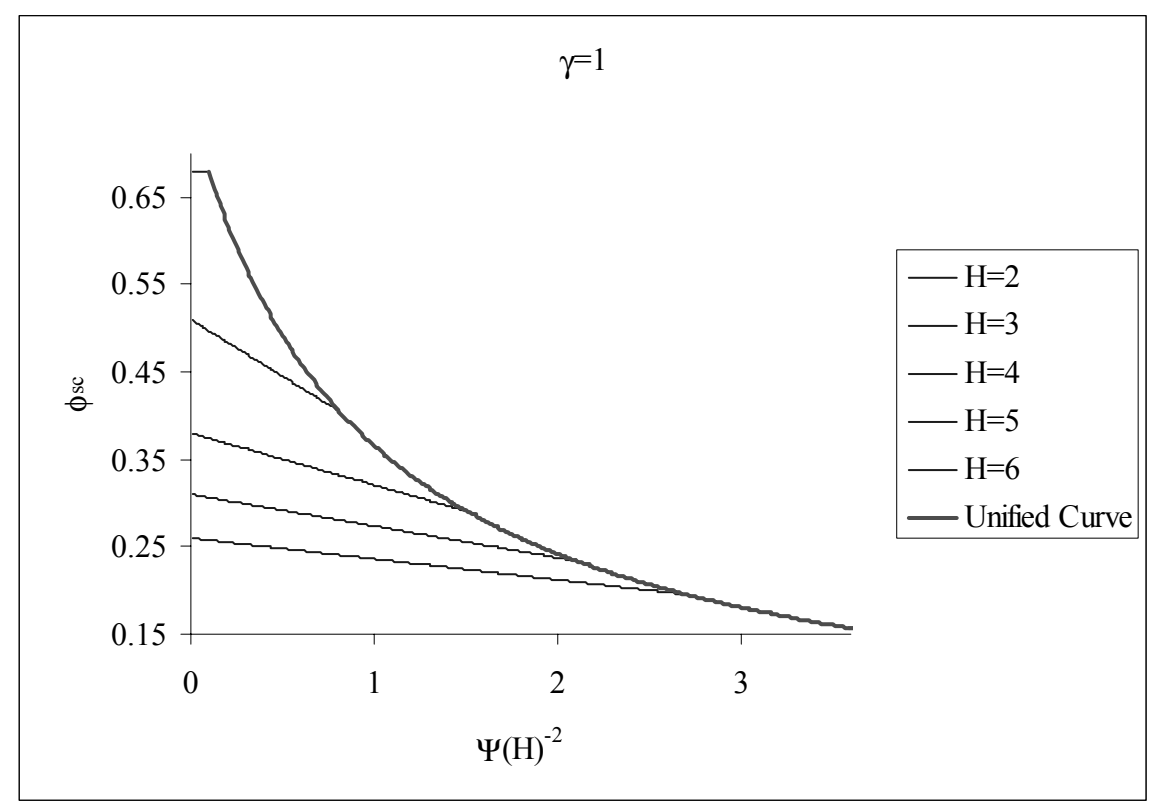

Figure 3.2 Shear Flow Factors in Distinct Form and Unified Curve Fit (Harp and Salant, 2001)

Table 3.1 Coefficients of Equations 3.12, 3.13 (Harp and Salant, 2001)

\begin{tabular}{|l|c|c|}
\hline$\gamma$ & $\mathrm{c}_{1}$ & $\mathrm{r}$ \\
\hline \hline $1 / 9$ & 1.714 & 0.43 \\
$1 / 3$ & 1.284 & 0.44 \\
1 & 0.611 & 0.54 \\
3 & 0.186 & 1.33 \\
9 & 1.078 & 1.64 \\
\hline
\end{tabular}

Table 3.2 Coefficients of Equation 3.16 (Harp and Salant, 2001)

\begin{tabular}{|l|c|c|}
\hline$\gamma$ & $\mathrm{a}_{1}$ & $\mathrm{~b}_{1}$ \\
\hline \hline $1 / 9$ & 0.896 & 2.121 \\
$1 / 3$ & 0.990 & 1.706 \\
1 & 1.334 & 1.400 \\
3 & 1.936 & 1.554 \\
9 & 4.762 & 1.443 \\
\hline
\end{tabular}

Table 3.3 Coefficients of Equation 3.17 (Harp and Salant, 2001)

\begin{tabular}{|l|c|c|}
\hline$\gamma$ & $\mathrm{a}_{2}$ & $\mathrm{~b}_{2}$ \\
\hline \hline $1 / 9$ & 0.926 & 0.005 \\
$1 / 3$ & 0.686 & 0.019 \\
1 & 0.657 & 0.081 \\
3 & 0.730 & 0.503 \\
9 & 0.927 & 3.704 \\
\hline
\end{tabular}


Table 3.4 Coefficients of Equation 3.15

(Harp and Salant, 2001)

\begin{tabular}{|c|c|c|c|c|}
\hline$\gamma$ & $\mathrm{H}$ & $\mathrm{m}$ & $\mathrm{c}_{2}$ & Range \\
\hline \hline $1 / 9$ & 2 & 0 & 1.10 & $\Psi\left(\mathrm{H}^{-2}\right)<0.01$ \\
$1 / 9$ & 3 & 0.4715 & 0.95 & $\Psi\left(\mathrm{H}^{-2}\right)<0.1$ \\
$1 / 9$ & 4 & 0.4164 & 0.74 & $\Psi\left(\mathrm{H}^{-2}\right)<0.4$ \\
$1 / 9$ & 5 & 0.2053 & 0.55 & $\Psi\left(\mathrm{H}^{-2}\right)<0.8$ \\
$1 / 9$ & 6 & 0.1227 & 0.45 & $\Psi\left(\mathrm{H}^{-2}\right)<1.0$ \\
\hline $1 / 3$ & 2 & 0 & 0.98 & $\Psi\left(\mathrm{H}^{-2}\right)<0.02$ \\
$1 / 3$ & 3 & 0.5051 & 0.80 & $\Psi\left(\mathrm{H}^{-2}\right)<0.4$ \\
$1 / 3$ & 4 & 0.2291 & 0.60 & $\Psi\left(\mathrm{H}^{-2}\right)<1.0$ \\
$1 / 3$ & 5 & 0.1217 & 0.47 & $\Psi\left(\mathrm{H}^{-2}\right)<1.3$ \\
$1 / 3$ & 6 & 0.0782 & 0.39 & $\Psi\left(\mathrm{H}^{-2}\right)<1.7$ \\
\hline 1 & 2 & 0 & 0.68 & $\Psi\left(\mathrm{H}^{-2}\right)<0.1$ \\
1 & 3 & 0.1295 & 0.51 & $\Psi\left(\mathrm{H}^{-2}\right)<0.8$ \\
1 & 4 & 0.0593 & 0.38 & $\Psi\left(\mathrm{H}^{-2}\right)<1.5$ \\
1 & 5 & 0.0363 & 0.31 & $\Psi\left(\mathrm{H}^{-2}\right)<2.1$ \\
1 & 6 & 0.0239 & 0.26 & $\Psi\left(\mathrm{H}^{-2}\right)<2.7$ \\
\hline 3 & 2 & 0 & 0.37 & $\Psi\left(\mathrm{H}^{-2}\right)<0.5$ \\
3 & 3 & 0.0341 & 0.26 & $\Psi\left(\mathrm{H}^{-2}\right)<2.3$ \\
3 & 4 & 0.0110 & 0.18 & $\Psi\left(\mathrm{H}^{-2}\right)<3.2$ \\
3 & 5 &. .0084 & 0.15 & $\Psi\left(\mathrm{H}^{-2}\right)<4.5$ \\
3 & 6 &. .0046 & 0.12 & $\Psi\left(\mathrm{H}^{-2}\right)<5.6$ \\
\hline 9 & 2 & 0 & 0.21 & $\Psi\left(\mathrm{H}^{-2}\right)<0.1$ \\
9 & 3 & 0.0059 & 0.11 & $\Psi\left(\mathrm{H}^{-2}\right)<6$ \\
9 & 4 & 0.0029 & 0.08 & $\Psi\left(\mathrm{H}^{-2}\right)<12$ \\
9 & 5 & 0.0015 & 0.06 & $\Psi\left(\mathrm{H}^{-2}\right)<21$ \\
9 & 6 & 0.0010 & 0.05 & $\Psi\left(\mathrm{H}^{-2}\right)<27$ \\
\hline
\end{tabular}

The Peklenik number $\gamma$ describes the shape and directionality of the surface asperities by using the ratio of asperity length in the $\mathrm{x}$-direction to that in the y-direction as shown in Figure 3.3. A Peklenik number of 1 describes an isotropic surface that is symmetric and has no directionality. Surfaces possessing Peklenik numbers greater than 
one or less than one contain asperities that are longer in the $\mathrm{x}$ - or $\mathrm{y}$-direction, respectively.

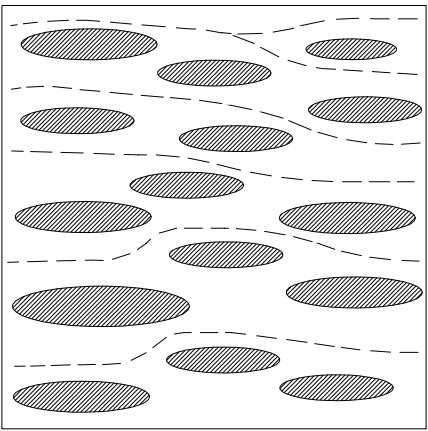

$$
\gamma>1
$$

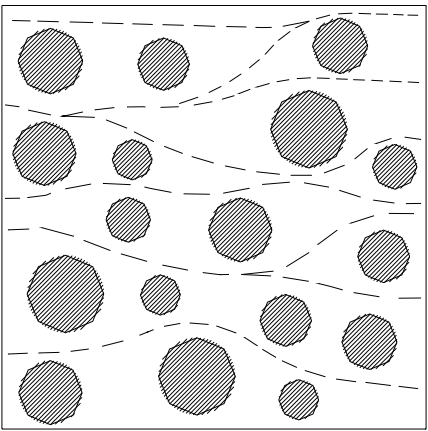

$$
\gamma=1
$$
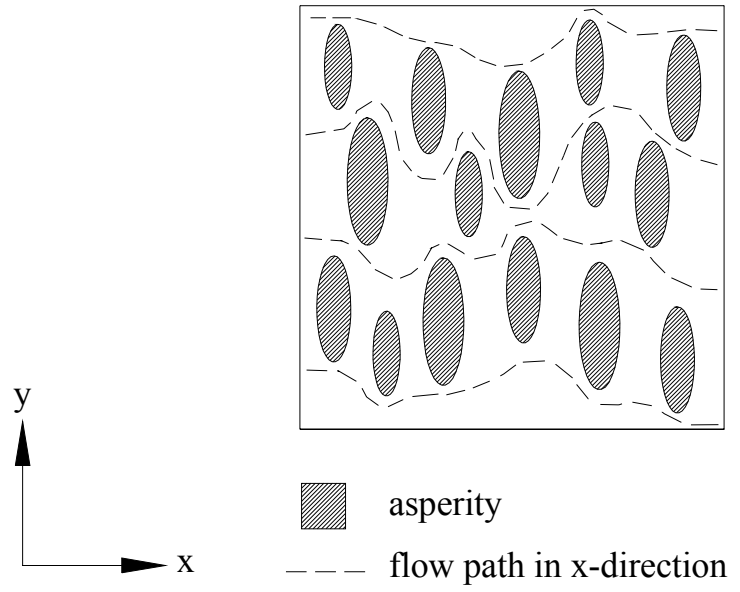

$$
\gamma<1
$$

Figure 3.3 Various Asperity Orientations as defined by $\gamma$. 
The parameter $\Psi$ aids in the prediction of cavitation based on the observation that the effects of cavitaion increase with increasing fluid viscosity, increasing sliding speed, increasing asperity width, decreasing roughness, and decreasing pressure relative to the cavitation pressure. It is defined by,

$$
\Psi=\frac{\mu U \lambda_{x}}{\left(\bar{p}-p_{c}\right) \sigma^{2}}
$$

Where $\lambda_{\mathrm{x}}$ is the autocorrelation length in the $\mathrm{x}$-direction, and $\bar{p}$ is an estimate of

the pressure midway between two neighboring grid points, found by taking the average of the pressure values at those points. The shear and density flow factors are functions of $\Psi$; as the cavitation number increases, the shear flow factors decrease and the density flow factors increase.

\subsubsection{Interpolation and Extrapolation of Shear Flow Factors}

As shown in Table 3.4, the coefficients of Equation 3.15 are not constant. These coefficients were determined in past numerical experiments and are available as data for this study for the twenty-five values of $\mathrm{H}$ and $\gamma$ specified in the table.

For the full extent of this study, the evaluation of flow factors for any value of $\mathrm{H}$ is necessary, therefore, an interpolation program was written to calculate the unknown shear flow factor coefficients for all film thicknesses that fall between the values listed in Table 3.4. Cubic spline interpolation proved to be the most accurate method of finding the two coefficients for each equation. Additionally, the point in which the distinct linear 
equation joins the unified curve in the $\Psi(\mathrm{H})^{-2}$ range must be determined in order for the proper equation for $\phi_{\text {s.c }}$ to be utilized. This is also accomplished in the interpolation program. Figure 3.4 shows an example of several interpolated flow factor curves for film thickness values between 3 and 4 and the points at which they intersect the unified curve.

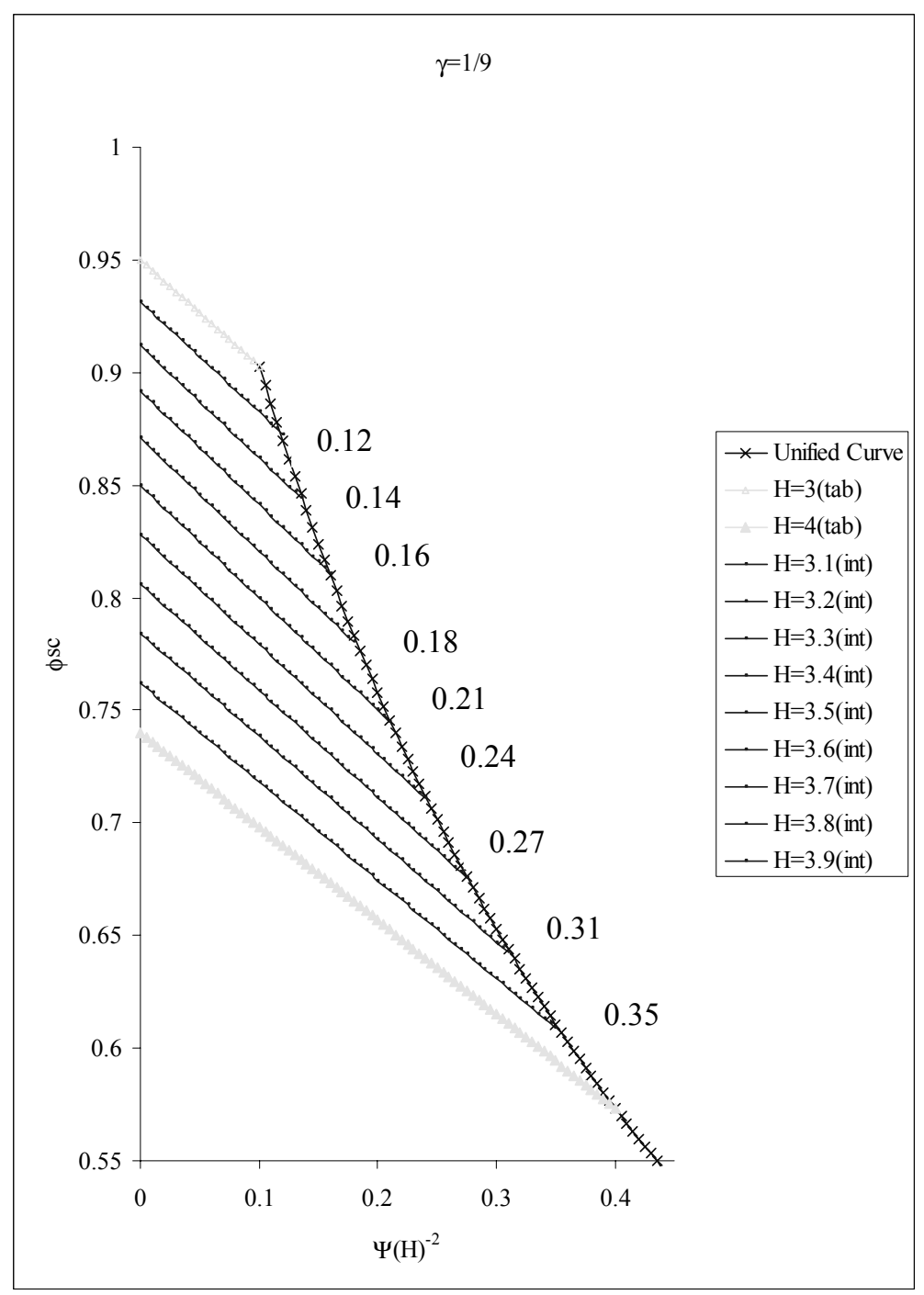

Figure 3.4 Interpolated Shear Flow Factor Values between $\mathrm{H}=3$ and 4 For $\gamma=1 / 9$, Showing Points of Intersection with the Unified Curve. 
Additionally, the shear flow factor equations from Harp (2001) were determined for a range of film thicknesses $2.00 \leq \mathrm{H} \leq 6.00$. In this present work, the range of values of $\mathrm{H}$ is extended to include $1.00 \leq \mathrm{H}<2.00$, and an extrapolation program was written to facilitate this for the calculation of the shear flow factors. The relevant equations for the changes are shown in Equations 3.19 - 3.21. The coefficients for these equations are shown in Tables $3.5-3.7$.

$\phi_{s . c}(H, \gamma, \Psi)=-m \Psi(H)^{-2}+\mathrm{c}_{2}\left(\right.$ for $\Psi(H)^{-2}$ range in Table 3.5)

$\phi_{s . c}(H, \gamma, \Psi)=\frac{1}{a_{3}+b_{3}\left[\Psi(H)^{-2}\right]^{0.5}}\left(\right.$ for $\Psi(H)^{-2}$ range in Table 3.6)

$\phi_{s . c}(H, \gamma, \Psi)=\frac{1}{a_{1}+b_{1} \Psi(H)^{-2}}\left(\right.$ for $\Psi(H)^{-2} \geq$ upper limit in Table 3.7)

Table 3.5 Coefficients of Equation 3.19

\begin{tabular}{|c|c|c|l|l|}
\hline$\gamma$ & $\mathrm{H}$ & $\mathrm{m}$ & $\mathrm{c}_{2}$ & Range \\
\hline \hline $1 / 9$ & 1 & 0 & 1.247 & $\Psi\left(\mathrm{H}^{-2}\right)<7.03 \times 10^{-9}$ \\
$1 / 3$ & 1 & 0 & 1.172 & $\Psi\left(\mathrm{H}^{-2}\right)<7.61 \times 10^{-8}$ \\
1 & 1 & 0 & 0.867 & $\Psi\left(\mathrm{H}^{-2}\right)<1.26 \times 10^{-11}$ \\
3 & 1 & 0 & 0.478 & $\Psi\left(\mathrm{H}^{-2}\right)<0.10$ \\
9 & 1 & 0 & 0.331 & $\Psi\left(\mathrm{H}^{-2}\right)<2.18 \times 10^{-9}$ \\
\hline
\end{tabular}

Table 3.6 Coefficients of Equation 3.20

\begin{tabular}{|r|l|l|l|l|}
\hline \multicolumn{1}{|c|}{$\gamma$} & $\mathrm{H}$ & $\mathrm{a}_{3}$ & $\mathrm{~b}_{3}$ & Range \\
\hline \hline $1 / 9$ & 1 & 0.802 & 0.899 & $7.03 \times 10^{-9} \leq \Psi\left(\mathrm{H}^{-2}\right)<0.03$ \\
& & & & \\
$1 / 3$ & 1 & 0.853 & 0.968 & $7.61 \times 10^{-8} \leq \Psi\left(\mathrm{H}^{-2}\right)<0.07$ \\
1 & 1 & 1.153 & 1.007 & $1.26 \times 10^{-11} \leq \Psi\left(\mathrm{H}^{-2}\right)<0.12$ \\
3 & 1 & & & \\
9 & 1 & 3.021 & 3.171 & $2.18 \times 10^{-9} \leq \Psi\left(\mathrm{H}^{-2}\right)<1.15$ \\
\hline
\end{tabular}


Table 3.7 Coefficients of Equation 3.21

\begin{tabular}{|c|l|l|}
\hline$\gamma$ & $\mathrm{a}_{1}$ & $\mathrm{~b}_{1}$ \\
\hline \hline $1 / 9$ & 0.896 & 2.121 \\
$1 / 3$ & 0.990 & 1.706 \\
1 & 1.334 & 1.400 \\
3 & 1.936 & 1.554 \\
9 & 4.762 & 1.443 \\
\hline
\end{tabular}

Since this work includes computation of shear deformations, it was necessary to compute the viscous shear stress. This was done using the shear stress flow factor calculations from Patir and Cheng (1979), neglecting the effect of cavitation. The average dimensionless shear stress in the $\mathrm{x}$-direction is given by,

$$
\hat{\tau}_{a v g}=\frac{\tau_{a v g}}{E}=\frac{\mu U}{H \sigma E}\left(\phi_{f}+\phi_{f s s}\right)+\phi_{f p p} \frac{H \sigma}{2 E} \frac{\partial P\left(p_{a}-p_{c}\right)}{\partial \hat{x} L_{x}}
$$

The $\phi_{f}$ term results from averaging the sliding velocity component of the shear stress. It is obtained from the previous studies of Patir and Cheng (1979) through integration for any given frequency density of roughness heights with the following two equations,

for $H \leq 3$ :

$$
\begin{aligned}
& \phi_{f}=\frac{35}{32} z\left\{\left(1-z^{2}\right)^{3} \ln [300(z+1)]\right\}+ \\
& \frac{35}{32} z\left\{\frac{[-55+z(132+z(345+z(-160+z(-405+z(60+147 z)))))]}{60}\right\}
\end{aligned}
$$


for $H>3$ :

$$
\phi_{f}=\frac{35}{32} z\left\{\left(1-z^{2}\right)^{3} \ln \frac{z+1}{z-1}+\frac{z}{15}\left[66+z^{2}\left(30 z^{2}-80\right)\right]\right\}
$$

The $\phi_{\mathrm{fp}}$ term is a correction factor for the mean pressure flow component of the shear stress and is a function of $\mathrm{H}$ and the surface pattern parameter, $\gamma$. All $\phi_{\mathrm{fp}}$ values approach 1.0 asymptotically as $\mathrm{H}$ increases and are higher for longitudinally oriented surfaces and lower for transversely oriented surfaces. Data obtained from Patir and Cheng (1979) through simulation and fitted into empirical relations for $\theta=0$ and $90^{\circ}$ are of the form,

$\phi_{f p}=1-D e^{-s H}$

and for any arbitrary angle $\theta$,

$$
\phi_{f p p}=\cos ^{2} \theta \cdot\left(\phi_{f p}\right)_{\theta=0}+\sin ^{2} \theta \cdot\left(\phi_{f p}\right)_{\theta=90}
$$

with the coefficients D and s given in Table 3.8 as functions of $\gamma$.

Table 3.8 Coefficients of Equation 3.25

\begin{tabular}{|l|c|c|}
\hline$\gamma$ & $\mathrm{D}$ & $\mathrm{s}$ \\
\hline $1 / 9$ & 1.51 & 0.52 \\
\hline $1 / 3$ & 1.47 & 0.58 \\
\hline 1 & 1.40 & 0.66 \\
\hline 3 & 0.98 & 0.79 \\
\hline 9 & 0.73 & 0.91 \\
\hline
\end{tabular}


The term $\phi_{\mathrm{fs}}$ is another correction factor that results from the combined effect of roughness and sliding, similar to the shear flow factors from the hydrodynamics analysis. $\phi_{\mathrm{fs}}$ and $\Phi_{\mathrm{fs}}$ are related by data obtained from Patir and Cheng (1979) through simulation and fitted empirically for $\theta=0$ and $90^{\circ}$ as follows,

$\phi_{f s}=V_{r 1} \Phi_{f s}\left(H, \gamma_{1}\right)-V_{r 2} \Phi_{f s}\left(H, \gamma_{2}\right)$

and for any arbitrary angle $\theta$,

$\phi_{f s s}=\sin \theta \cos \theta \cdot\left[\left(\phi_{f s}\right)_{\theta=90}-\left(\phi_{f s}\right)_{\theta=0}\right]$

with,

$\Phi_{f s}=A_{4} H^{A 5} e^{-A 6 H+A 7 H^{2}}$

for two surfaces of different roughness. For the case of one smooth surface, there is no second term in Equation 3.27, $\mathrm{V}_{\mathrm{r}}=1.0$, and therefore $\phi_{\mathrm{fs}}=\Phi_{\mathrm{fs}}$. The coefficients of Equation 3.29 are given in Table 3.9 as functions of $\gamma$.

Table 3.9 Coefficients of Equation 3.29

\begin{tabular}{|l|l|l|l|l|}
\hline$\gamma$ & $\mathrm{A}_{4}$ & $\mathrm{~A}_{5}$ & $\mathrm{~A}_{6}$ & $\mathrm{~A}_{7}$ \\
\hline $1 / 9$ & 14.1 & 2.45 & 2.30 & 0.10 \\
\hline $1 / 3$ & 12.3 & 2.32 & 2.30 & 0.10 \\
\hline 1 & 11.1 & 2.31 & 2.38 & 0.11 \\
\hline 3 & 9.8 & 2.25 & 2.80 & 0.18 \\
\hline 9 & 8.7 & 2.15 & 2.97 & 0.18 \\
\hline
\end{tabular}




\subsection{Deformation Analysis}

In a preliminary hydrodynamic study, a constant film thickness was used in the Reynolds equation and the circumferential displacement of the lip surface was modeled (Salant and Rocke, 2004) with the following expressions,

$$
\begin{array}{ll}
\hat{\delta}=\alpha \zeta\left[\frac{2 \hat{y}}{\hat{y}_{b}}-\frac{\hat{y}^{2}}{\hat{y}_{b}^{2}}\right] & \text { for } \hat{y} \leq \hat{y}_{b} \\
\hat{\delta}=\alpha \zeta \frac{\left[1-2 \hat{y}_{b}+2 \hat{y} \hat{y}_{b}-\hat{y}^{2}\right]}{\left(1-\hat{y}_{b}\right)^{2}} & \text { for } \hat{y}>\hat{y}_{b}
\end{array}
$$

Figure 5 shows the resulting pattern on the lip surface and how the major axes of the asperities would be tangent to the displacement curve under dynamic conditions.

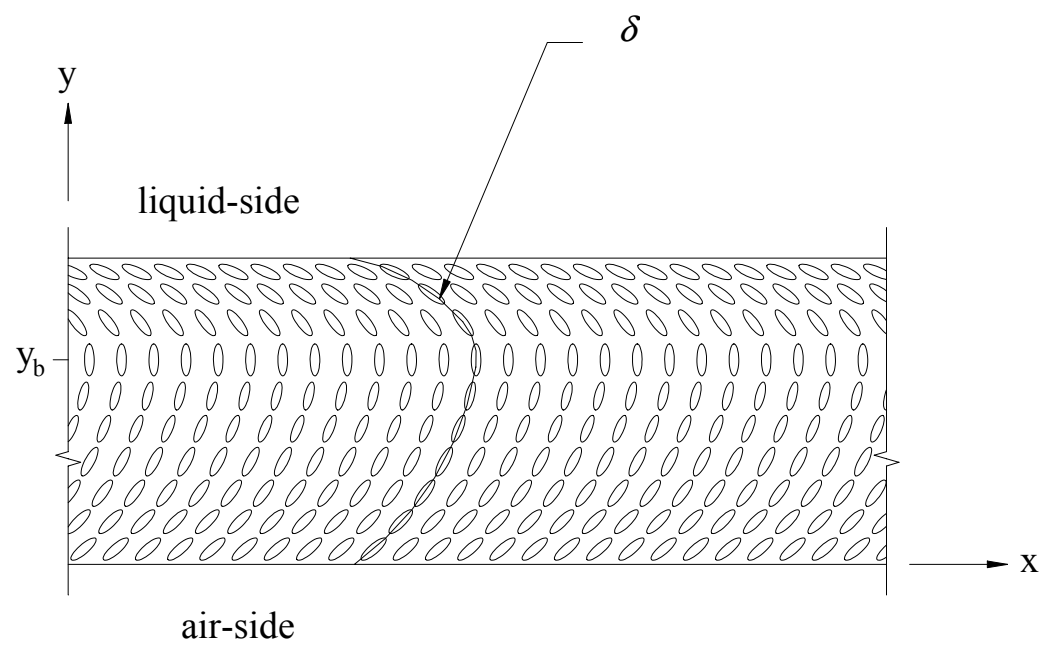

Figure 3.5 Distorted Asperity Pattern 
In the full elastohydrodynamic study, the film thickness and shear deformation are calculated. The latter is used in the asperity distortion analysis to compute the deformed asperity characteristics.

To compute the normal deformation of the lip, the influence coefficient matrix $\mathrm{I}_{1}$ is utilized such that the average film thickness (averaged in the x-direction) $\mathrm{H}$, can be expressed in discretized form (at the $i$ axial node) as,

$$
H=\hat{A}_{1}+\sum_{k=1}^{n}\left(I_{1}\right)_{i k}\left(\hat{p}_{\text {def }}-\hat{p}_{\text {contact }}\right)_{k}
$$

The dimensionless pressure $\hat{p}_{\text {def }}$ is related to the dimensionless pressure $\mathrm{P}$ in the fluid mechanics relations through,

$$
\hat{p}_{\text {def }}=P\left(p_{a}-p_{c}\right) / E+p_{c} / E
$$

To compute the shear deformation of the lip, an influence coefficient method characterized by the influence coefficient matrix $I_{2}$ is utilized. Thus, the circumferential displacement of the lip surface $\delta$ can be expressed in discretized form (at the $i$ axial node) as,

$$
\hat{\boldsymbol{\delta}}_{i}=\sum_{k=1}^{n}\left(I_{2}\right)_{i k}\left(\hat{\tau}_{\text {avg }}\right)_{k}
$$


The influence coefficients $\mathrm{I}_{1}$ and $\mathrm{I}_{2}$ and the contact pressure $\hat{p}_{\text {contact }}$ are obtained from a finite element analysis of a seal. This influence coefficient method has been used in previous lip seal studies (Salant and Flaherty, 1994; Shen and Salant, 2003).

\subsection{Finite Element Analysis}

A cross-section of the rubber material on a lip seal was measured and its dimensions and material properties were used to construct a model for the finite element analysis. Figure 3.6 shows the cross-section used for a two-dimensional axisymmetric analysis in ANSYS. The elastic modulus of the rubber is 3MPa and Poisson's ratio is 0.49 . The interference fit between the lip and the $38.3794 \mathrm{~mm}$ diameter shaft is $1.40 \mathrm{~mm}$.

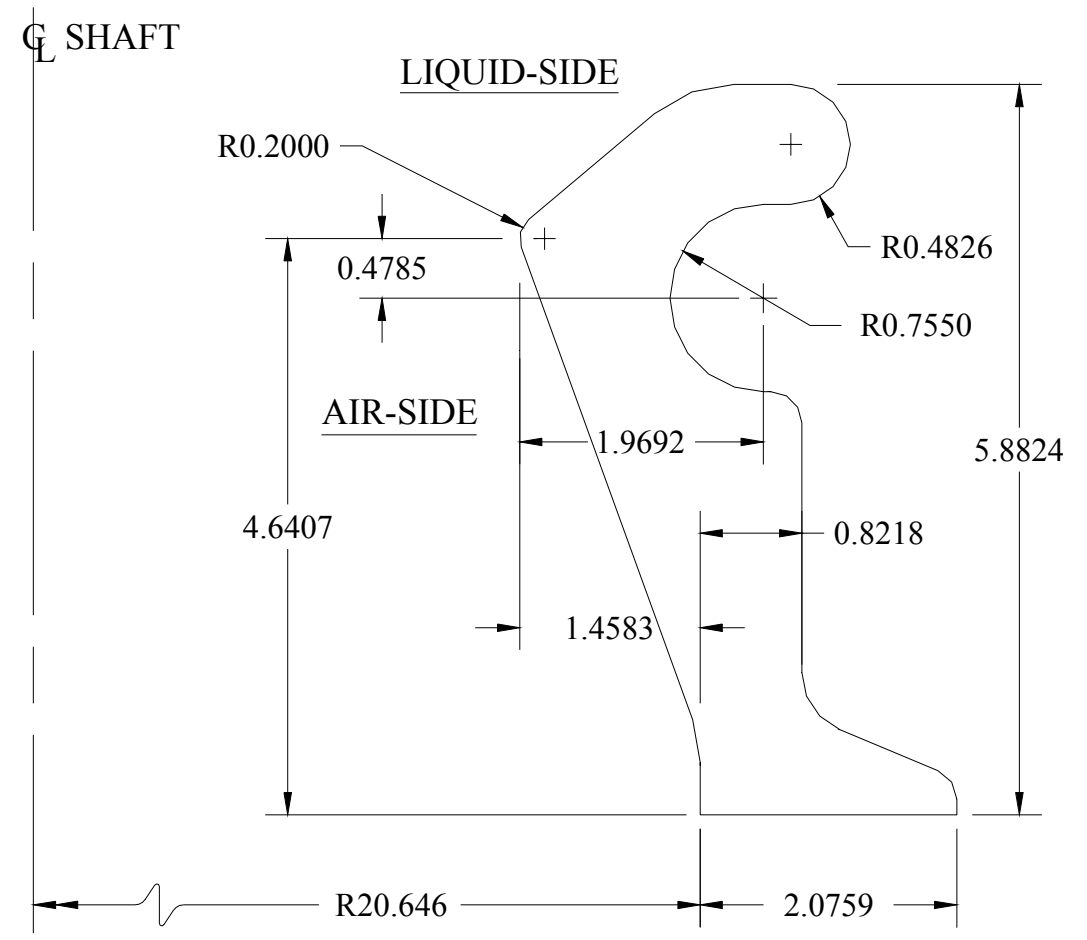

3.6 Detailed Cross-Section of Lip Seal Rubber (millimeters) 
Figure 3.7 shows the deformed results of the contact model. When the seal is mounted onto the shaft the angles formed on each side of the contact area are changed. These angles play an important role in successful lip seal behavior. The angle facing the air-side of the seal must be smaller than the angle facing the liquid-side. The difference in these angles and the location of the garter spring determine the contact pressure distribution under the lip and the deformation characteristics of the lip, which in turn, strongly affect lip seal behavior.

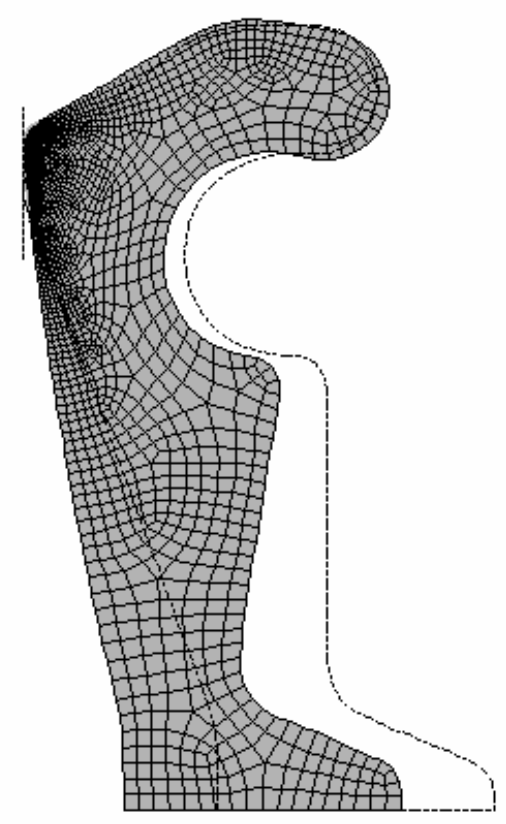

3.7 Deformed Results from Contact Model 
Initially, the model was constructed with a $0.01 \mathrm{~mm}$ radius in the area of the sealing zone, as it was not possible to obtain a precise measurement of that point. The resulting contact pressure for that model is shown in Figure 3.8. It is believed that during the run-in period, a small portion of the tip on a new seal wears off and leaves a slightly flattened region to function as the sealing band (Horve, 1996). The data in the figure support this, since it shows unrealisticly high contact pressure in the tip region, on the order of magnitude of the rubber's yield strength.

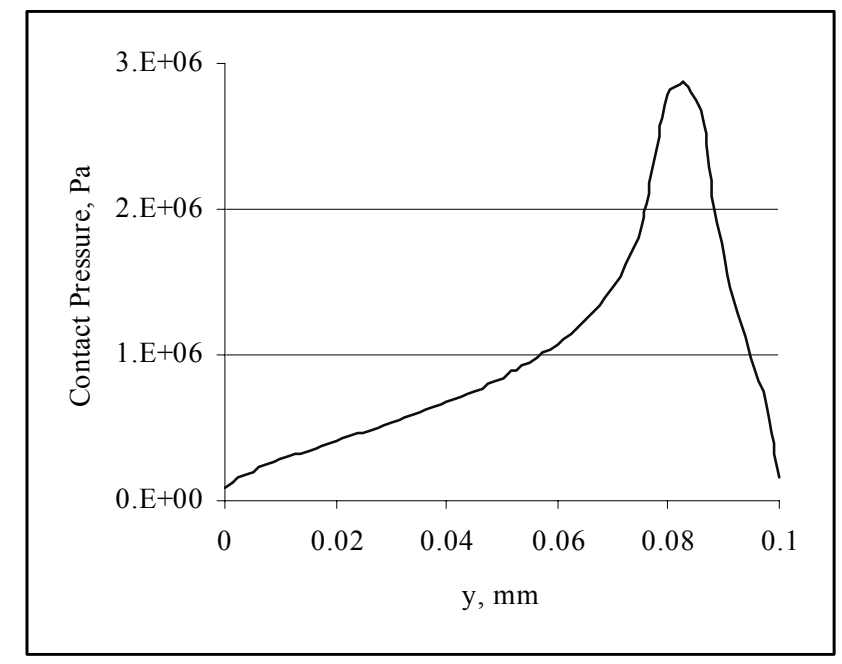

Figure 3.8 Contact Pressure Distribution before Wear

A more realistic model was constructed with a $0.2 \mathrm{~mm}$ tip radius to better simulate the more flattened out area of the lip's sealing zone. This is the model that is shown in Figures 3.6 and 3.7 and is used in the finite element analysis. The contact pressure distributioin for the selected model is more reasonable, based on previous experimental measurements (Müller, 1987), and is shown in Figure 5.18. 
In addition to the contact pressure, information regarding the influence of the flexibility of the rubber in the sealing zone is required from the analysis. Equations 3.32 and 3.34 show that the calculation of the normal and shear deformations require coefficient matrices $\mathrm{I}_{1}$ and $\mathrm{I}_{2}$. The $i, k$ term in the $\mathrm{I}_{1}$ matrix represents the normal deformation at node $i$ produced by a unit force applied to node $k$. Similarly, the $i, k$ term in the $\mathrm{I}_{2}$ matrix represents the shear deformation at node $i$ produced by a unit force applied to node $k$. Figures 3.9 and 3.10 show the three dimensional plots of data for $\mathrm{I}_{1}$ and $\mathrm{I}_{2}$, respectively.

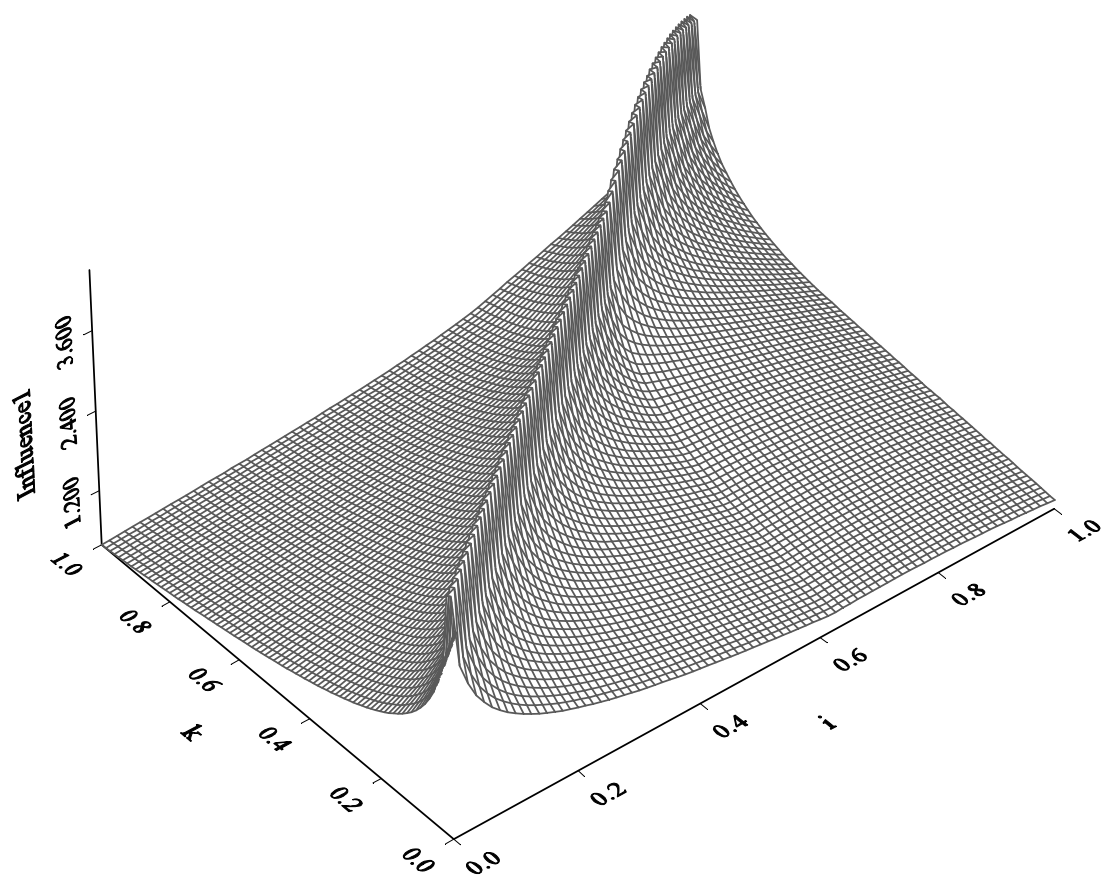

Figure 3.9 Influence Coefficient for Normal Deformations 


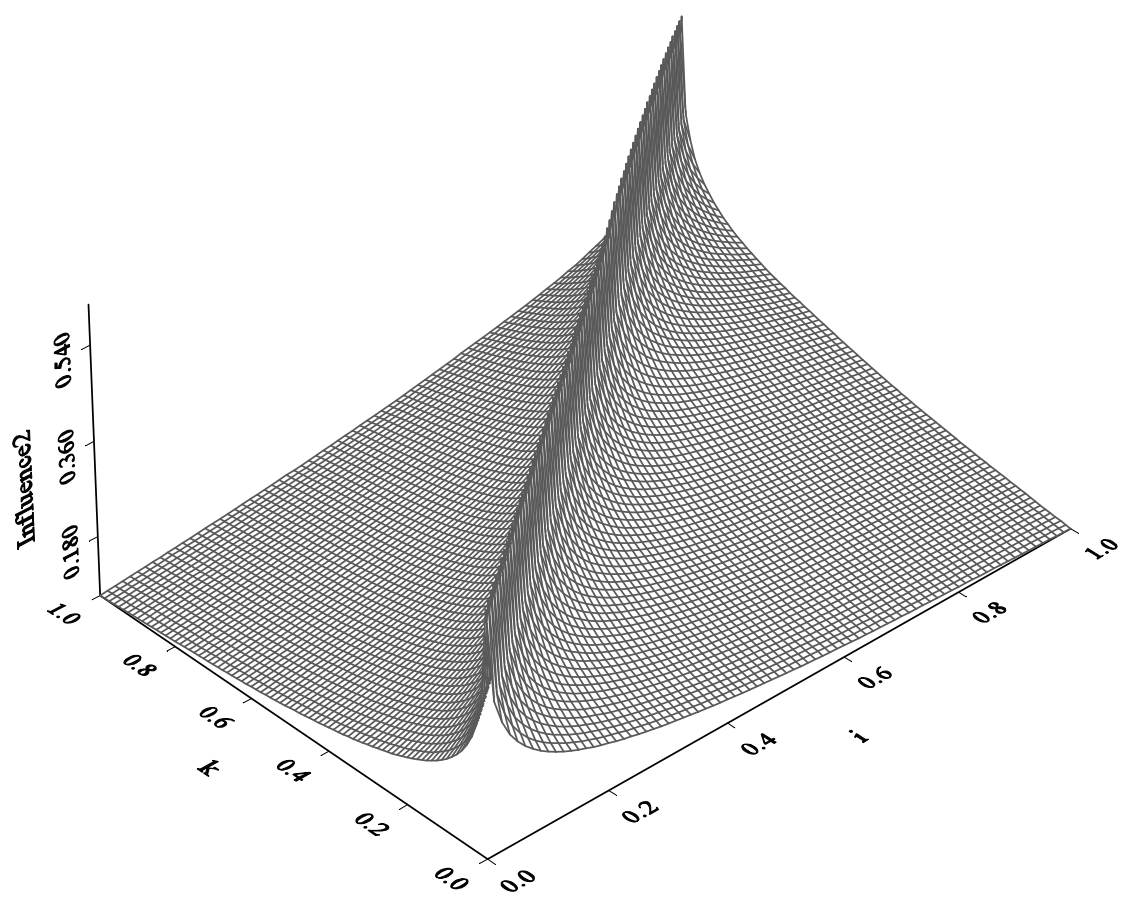

Figure 3.10 Influence Coefficient for Shear Deformations

\subsection{Asperity Distortion Analysis}

With the circumferential displacement $\delta$ of the lip surface known, the following method for modeling asperity distortion is used. Figure 3.11 shows an exaggerated diagram of how $\delta$ affects the orientation and aspect ratio of an asperity. These values are found by calculating the rotation of axes at every grid point on the solution domain. This allows for an analysis to be conducted for a surface consisting of any quantity of asperities of varying size and aspect ratio.

The following steps define the general model (Thomas, 1958) that will compute the distortion of either a circular or elliptical asperity on the lip surface as it experiences circumferential displacement. With the asperity autocorrelation length $\lambda_{Y}$ equal to the 
half major axis and $\lambda_{\mathrm{x}}$ equal to the half minor axis for the initial shape of an ellipse ( $\lambda_{\mathrm{x}}=$ $\lambda_{Y}$ if starting with a circular shape), the equation for an undeformed asperity is defined as,

$$
\frac{X^{2}}{\lambda_{X}^{2}}+\frac{Y^{2}}{\lambda_{Y}^{2}}=1
$$

As shown in Figure 3.11, asperity displacement is modeled as a function of $Y$ only and can therefore be defined with the equation,

$$
\lambda_{Y}^{2}\left[X^{*}-\delta(Y)\right]^{2}+\lambda_{X}^{2}\left[Y^{*}\right]^{2}=\lambda_{X}^{2} \lambda_{Y}^{2}
$$

where,

$$
\begin{aligned}
& X^{*}=X+\delta(Y) \\
& Y^{*}=Y
\end{aligned}
$$

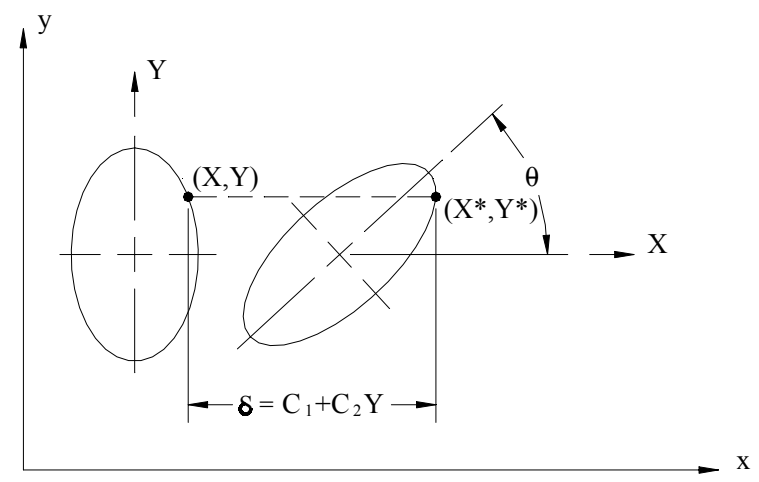

Figure 3.11 Asperity Distortion as Simulated with the Rotation of Axes 
In expanded form,

$\lambda_{Y}^{2}\left[X^{*}\right]^{2}-\lambda_{Y}^{2} 2 \delta(Y) X^{*}+\lambda_{Y}^{2}[\delta(Y)]^{2}+\lambda_{X}^{2} Y^{2}-\lambda_{X}^{2} \lambda_{Y}^{2}=0$

and letting

$\delta(Y)=C_{1}+C_{2} Y$

The equation,

$\lambda_{Y}^{2}\left[X^{*}\right]^{2}-2 C_{2} \lambda_{Y}^{2} X^{*} Y+\left(\lambda_{X}^{2}+C_{2}^{2} \lambda_{Y}^{2}\right) Y^{2}+2 C_{1} C_{2} \lambda_{Y}^{2} Y-2 C_{1} \lambda_{Y}^{2} X^{*}+C_{1}^{2} \lambda_{Y}^{2}-\lambda_{X}^{2} \lambda_{Y}^{2}=0$

takes the form of the general curve equation,

$A\left[X^{*}\right]^{2}+B X^{*} Y+C Y^{2}+D X^{*}+E Y+F+G=0$

with

$$
\begin{aligned}
& A=\lambda_{Y}^{2} \\
& B=-2 C_{2} \lambda_{Y}^{2} \\
& C=\lambda_{X}^{2}+C_{2}^{2} \lambda_{Y}^{2} \\
& D=-2 C_{1} \lambda_{Y}^{2} \\
& E=2 C_{1} C_{2} \lambda_{Y}^{2} \\
& F=C_{1}^{2} \lambda_{Y}^{2} \\
& G=-\lambda_{X}^{2} \lambda_{Y}^{2}
\end{aligned}
$$

A rotation of axes through an angle $\theta$ is performed in the counterclockwise direction to eliminate the cross-product term and results in the equations,

$$
\begin{aligned}
& X^{*}=X^{\prime} \cos \theta-Y^{\prime} \sin \theta \\
& Y^{*}=X^{\prime} \sin \theta+Y^{\prime} \cos \theta
\end{aligned}
$$


With new coefficients related to the old as follows:

$$
\begin{aligned}
& A^{\prime}=A \cos ^{2} \theta+B \cos \theta \sin \theta+C \sin ^{2} \theta \\
& B^{\prime}=B\left(\cos ^{2} \theta-\sin ^{2} \theta\right)+2(C-A) \sin \theta \cos \theta \\
& C^{\prime}=A \sin ^{2} \theta-B \sin \theta \cos \theta+C \cos ^{2} \theta \\
& D^{\prime}=D \cos \theta+E \sin \theta \\
& E^{\prime}=-D \sin \theta+E \cos \theta \\
& F^{\prime}=F \\
& G^{\prime}=G
\end{aligned}
$$

Setting $\mathrm{B}^{\prime}=0$ and solving for $\theta$, the angle of rotation (deformed asperity orientation) can be found that will eliminate the new cross-product term,

$$
\begin{aligned}
& 0=B\left(\cos ^{2} \theta-\sin ^{2} \theta\right)+2(C-A) \sin \theta \cos \theta \\
& 0=B \cos 2 \theta+(C-A) \sin 2 \theta \\
& \Rightarrow \cot 2 \theta=\frac{A-C}{B} \\
& \Rightarrow 2 \theta=\arctan \left[\frac{B}{A-C}\right] \\
& \Rightarrow \theta=\frac{1}{2} \arctan \left[\frac{B}{A-C}\right]=\frac{1}{2} \arctan \left[\frac{-2 C_{2}}{1-\left(1+C_{2}^{2}\right)}\right]=\frac{1}{2} \arctan \left[\frac{2}{C_{2}}\right]
\end{aligned}
$$

With new axes and coefficients defined, Equation 3.42 can be written in ellipse equation form,

$$
A^{\prime}\left[X^{*}+\frac{D^{\prime}}{2 A^{\prime}}\right]+C^{\prime}\left[Y+\frac{E^{\prime}}{2 C^{\prime}}\right]=\underbrace{-\left(F^{\prime}+G^{\prime}\right)+\frac{D^{\prime 2}}{4 A^{\prime}}+\frac{E^{\prime 2}}{4 C^{\prime}}}_{H}
$$


dividing both sides by $\mathrm{H}$,

$$
\frac{\left[X^{*}\right]^{2}}{\left[\frac{H}{A^{\prime}}\right]}+\frac{Y^{2}}{\left[\frac{H}{C^{\prime}}\right]}=1
$$

with

$$
\frac{H}{A^{\prime}}=a^{2}, \quad \frac{H}{C^{\prime}}=b^{2}
$$

Therefore, the asperity aspect ratio $(\gamma=\mathrm{a} / \mathrm{b})$ is found in the following manner:

$a=\left[\frac{-\left(F^{\prime}+G^{\prime}\right)+\frac{D^{\prime 2}}{4 A^{\prime}}+\frac{E^{\prime 2}}{4 C^{\prime}}}{A^{\prime}}\right]^{\frac{1}{2}}$

and

$b=\left[\frac{-\left(F^{\prime}+G^{\prime}\right)+\frac{D^{\prime 2}}{4 A^{\prime}}+\frac{E^{\prime 2}}{4 C^{\prime}}}{C^{\prime}}\right]^{\frac{1}{2}}$

Expressions for $\mathrm{X}^{\prime}$ and $\mathrm{Y}^{\prime}$ are found using Equations (3.37), (3.38), (3.40), (3.43) and (3.44) with the following substitutions,

$$
\begin{aligned}
& X^{*}=X^{\prime} \cos \theta-Y^{\prime} \sin \theta \\
& Y^{*}=X^{\prime} \sin \theta+Y^{\prime} \cos \theta \\
& X^{*}=X+\delta(Y)=X+C_{1}+C_{2} Y \\
& Y^{*}=Y
\end{aligned}
$$


therefore,

$$
\begin{aligned}
& X^{\prime} \cos \theta-Y^{\prime} \sin \theta=X+C_{1}+C_{2} Y \\
& X^{\prime} \sin \theta+Y^{\prime} \cos \theta=Y
\end{aligned}
$$

Solving simultaneously for $\mathrm{X}^{\prime}$ and $\mathrm{Y}^{\prime}$,

$$
\begin{gathered}
X^{\prime}=\frac{(\cos \theta)\left(X+C_{1}+C_{2} Y\right)-(-\sin \theta)(Y)}{(\cos \theta)(\cos \theta)-(-\sin \theta)(\sin \theta)} \\
Y^{\prime}=\frac{(\cos \theta)(Y)-(\sin \theta)\left(X+C_{1}+C_{2} Y\right)}{(\cos \theta)(\cos \theta)-(-\sin \theta)(\sin \theta)}
\end{gathered}
$$

Substituting Equations 3.59 and 3.60 into Equations 3.43 and 3.44, transformed points $\mathrm{X}^{*}$ and $\mathrm{Y}^{*}$ and orientation angle $\theta$ can be found for a rotated ellipse given an original circle or ellipse and the constants $C_{1}$ and $C_{2}$. Figures $3.12 \mathrm{a}$ and $3.12 \mathrm{~b}$ show an example of the transformation of an undeformed ellipse to a deformed ellipse using the rotation of axes equations. Figure $3.12 \mathrm{c}$ verifies that the same ellipse is created when using $\mathrm{X}^{*}=\mathrm{X}+\delta$ and $\mathrm{Y}^{*}=\mathrm{Y}$.

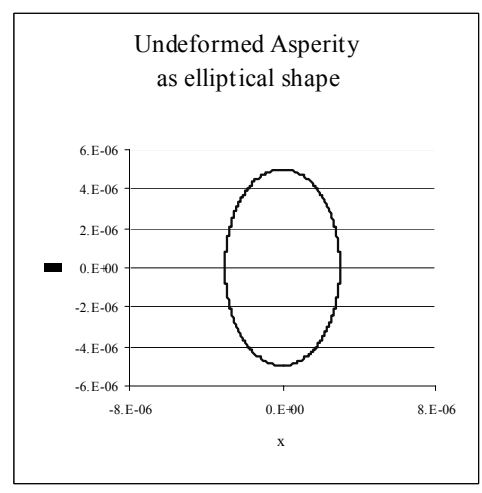

(a)

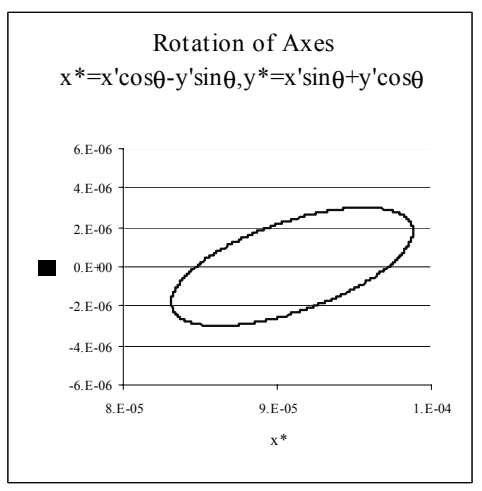

(b)

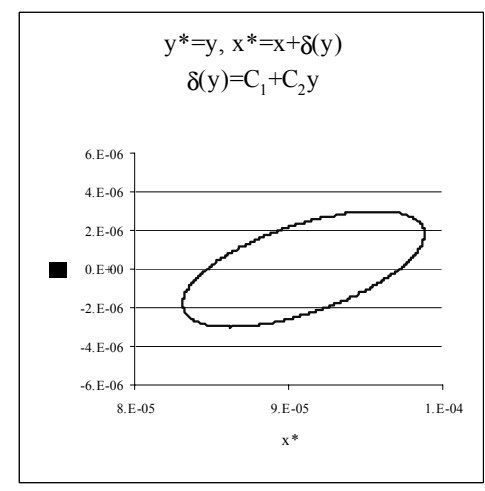

(c)

Figure 3.12 Transformation using rotation of axes (b) and $\mathrm{X}+\delta(\mathrm{Y}), \mathrm{Y}(\mathrm{c})$ 
$C_{2}$ is calculated in the FORTRAN program as the slope of the $\delta(y)$ curve at every

nodal point, and $C_{1}$ is found with,

$C_{1}=\delta-C_{2} y$

and substituting dimensionless terms,

$$
\hat{\delta}=\frac{\delta}{L_{x}} \quad \hat{y}=\frac{y}{L_{y}}
$$

Equation 3.61 can be written,

$$
C_{1}=\hat{\delta} L_{x}-C_{2} \hat{y} L_{y}
$$

The rotation angle $\theta$ and aspect ratio $(\gamma=a / b)$ of asperities at every nodal point is found using Equations 3.52, 3.55, and 3.56 respectively. 


\section{CHAPTER IV \\ NUMERICAL SCHEME}

\subsection{Discretization of Reynolds Equation}

A closed form solution for the Reynolds equation is not possible; therefore, a numerical finite difference method is utilized. The selected discretizatioin method is a control-volume formulation described in detail by Patankar (1980). It implies integral conservation of mass and momentum between neighboring grid points throughout the solution space.

The solution space is divided into individual control volumes as shown in Figure 4.1. The pressure grid points are located at the center of the control volumes.

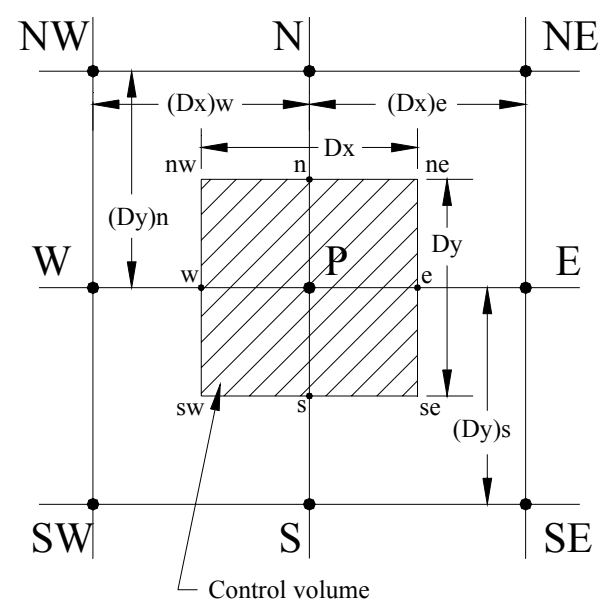

Figure 4.1 Diagram of the Control Volume for the Discretization Process 
Equation 3.5 is discretized by considering the layout of Figure 4.1. Surrounding nodes are labeled according to their directional location from the center point of interest. The boundaries of the control volume are labeled in the same manner, but with lower case letters.

Integrating Equation 3.5 over a control volume in the flow field from $\hat{x}_{w}$ to $\hat{x}_{e}$ and $\hat{y}_{s}$ to $\hat{y}_{n}$ produces,

$$
\begin{aligned}
& \int_{\hat{y}_{s}}^{\hat{y}_{y_{w}} \hat{x}_{e}} \frac{\partial}{\partial \hat{x}}\left(H^{3} \phi_{x x} \frac{\partial P}{\partial \hat{x}}\right) d x d y+\mathrm{K} \int_{\hat{y}_{s}}^{\hat{y}_{n}} \int_{\hat{x}_{w}}^{\hat{x}_{e}} \frac{\partial}{\partial \hat{x}}\left(H^{3} \phi_{x y} \frac{\partial P}{\partial \hat{y}}\right) d x d y+\mathrm{K}^{2} \int_{\hat{x}_{w}}^{\hat{x}_{y_{y}} \hat{y}_{p}} \frac{\partial}{\partial \hat{y}}\left(H^{3} \phi_{y y} \frac{\partial P}{\partial \hat{y}}\right) d y d x \\
& +\mathrm{K} \int_{\hat{x}_{w} \hat{y}_{s}}^{\hat{x}_{e} \hat{y}_{n}} \frac{\partial}{\partial \hat{y}}\left(H^{3} \phi_{y x} \frac{\partial P}{\partial \hat{x}}\right) d y d x=6 \zeta \int_{\hat{y}_{s} \hat{x}_{w}}^{\hat{y}_{n} \hat{x}_{e}} \frac{\partial}{\partial \hat{x}}\left(H-\phi_{c . c}\right) d x d y+ \\
& 6 \zeta \int_{\hat{y}_{s} \hat{x}_{w}}^{\hat{y}_{n} \hat{x}_{e}}\left(\frac{\partial \phi_{s . c . x}}{\partial \hat{x}}\right) d x d y+6 \zeta \mathrm{K} \int_{\hat{x}_{w} \hat{y}_{s}}^{\hat{x}_{e} \hat{y}_{n}}\left(\frac{\partial \phi_{\text {s.c. } y}}{\partial \hat{y}}\right) d y d x
\end{aligned}
$$

The first term in the left hand side of the equation becomes,

$$
\begin{aligned}
& {\left[\left(H^{3} \phi_{x x} \frac{\partial P}{\partial \hat{x}}\right)_{e}-\left(H^{3} \phi_{x x} \frac{\partial P}{\partial \hat{x}}\right)_{W}\right] \Delta y} \\
& =\frac{2\left(H_{E}^{3} \phi_{x x E}\right)\left(H_{P}^{3} \phi_{x x P}\right)}{\left(H_{E}^{3} \phi_{x x E}+H_{P}^{3} \phi_{x x P}\right)} \frac{\Delta y}{\Delta x}\left(P_{E}-P_{P}\right)-\frac{2\left(H_{W}^{3} \phi_{x x W}\right)\left(H_{P}^{3} \phi_{x x P}\right)}{\left(H_{W}^{3} \phi_{x x W}+H_{P}^{3} \phi_{x x P}\right)} \frac{\Delta y}{\Delta x}\left(P_{P}-P_{W}\right)
\end{aligned}
$$


The second term in the left hand side of the equation becomes,

$$
\begin{aligned}
& {\left[\left(H^{3} \phi_{x y} \frac{\partial P}{\partial \hat{y}}\right)_{e}-\left(H^{3} \phi_{x y} \frac{\partial P}{\partial \hat{y}}\right)_{w}\right] \mathrm{K} \Delta y} \\
& =\frac{\mathrm{K}}{4} \frac{2\left(H_{E}^{3} \phi_{x y E}\right)\left(H_{P}^{3} \phi_{x y P}\right)}{\left(H_{E}^{3} \phi_{x y E}+H_{P}^{3} \phi_{x y P}\right)}\left(P_{N}+P_{E N}-P_{S}-P_{S E}\right) \\
& -\frac{\mathrm{K}}{4} \frac{2\left(H_{W}^{3} \phi_{x y W}\right)\left(H_{P}^{3} \phi_{x y P}\right)}{\left(H_{W}^{3} \phi_{x y W}+H_{P}^{3} \phi_{x y P}\right)}\left(P_{N W}+P_{N}-P_{S W}-P_{S}\right)
\end{aligned}
$$

The third term in the left hand side of the equation becomes,

$$
\begin{aligned}
& {\left[\left(H^{3} \phi_{y y} \frac{\partial P}{\partial \hat{y}}\right)_{n}-\left(H^{3} \phi_{y y} \frac{\partial P}{\partial \hat{y}}\right)_{s}\right] \mathrm{K}^{2} \Delta x} \\
& =\mathrm{K}^{2} \frac{2\left(H_{N}^{3} \phi_{y y N}\right)\left(H_{P}^{3} \phi_{y y P}\right)}{\left(H_{N}^{3} \phi_{y y N}+H_{P}^{3} \phi_{y y P}\right)} \frac{\Delta x}{\Delta y}\left(P_{N}-P_{P}\right)-\mathrm{K}^{2} \frac{2\left(H_{S}^{3} \phi_{y y S}\right)\left(H_{P}^{3} \phi_{y y P}\right)}{\left(H_{S}^{3} \phi_{y y S}+H_{P}^{3} \phi_{y y P}\right)} \frac{\Delta x}{\Delta y}\left(P_{P}-P_{S}\right)
\end{aligned}
$$

The fourth term in the left hand side of the equation becomes,

$$
\begin{aligned}
& {\left[\left(H^{3} \phi_{y x} \frac{\partial P}{\partial \hat{x}}\right)_{n}-\left(H^{3} \phi_{y x} \frac{\partial P}{\partial \hat{x}}\right)_{w}\right] \mathrm{K} \Delta x} \\
& =\frac{\mathrm{K}}{4} \frac{2\left(H_{N}^{3} \phi_{y x N}\right)\left(H_{P}^{3} \phi_{y x P}\right)}{\left(H_{N}^{3} \phi_{y x N}+H_{P}^{3} \phi_{y x P}\right)}\left(P_{E}+P_{E N}-P_{N W}-P_{W}\right) \\
& -\frac{\mathrm{K}}{4} \frac{2\left(H_{S}^{3} \phi_{y x S}\right)\left(H_{P}^{3} \phi_{y x P}\right)}{\left(H_{S}^{3} \phi_{y x S}+H_{P}^{3} \phi_{x y P}\right)}\left(P_{E}+P_{S E}-P_{W}-P_{S W}\right)
\end{aligned}
$$


The first term in the right hand side of the equation becomes,

$$
\begin{aligned}
& 6 \zeta\left[\left(H-\phi_{c . c}\right)_{e}-\left(H-\phi_{c . c}\right)_{w}\right] \Delta y \\
& =6 \zeta\left[\left(\frac{2\left(H_{P}\right)\left(H_{E}\right)}{\left(H_{P}+H_{E}\right)}-\frac{2\left(H_{P}\right)\left(H_{W}\right)}{\left(H_{P}+H_{W}\right)}\right)+\left(\frac{\phi_{c . c W}-\phi_{c . c E}}{2}\right)\right] \Delta y
\end{aligned}
$$

The second term in the right hand side of the equation becomes,

$$
\begin{aligned}
& 6 \zeta\left[\left(\phi_{\text {s.c. } x}\right)_{e}-\left(\phi_{\text {s.c.x }}\right)_{w}\right] \Delta y \\
= & 6 \zeta\left[\frac{\phi_{\text {s.c. } x E}-\phi_{\text {s.c. } x W}}{2}\right] \Delta y
\end{aligned}
$$

The third term in the right hand side of the equation becomes,

$$
\begin{aligned}
& 6 \zeta \mathrm{K}\left[\left(\phi_{\text {s.c.y }}\right)_{n}-\left(\phi_{\text {s.c.y }}\right)_{s}\right] \Delta x \\
& =6 \zeta \mathrm{K}\left[\frac{\phi_{\text {s.c.yN }}-\phi_{\text {s.c.yS }}}{2}\right] \Delta x
\end{aligned}
$$

Substituting Equations 4.2 through 4.8 into Equation 4.1 gives,

$$
\begin{aligned}
& A_{E 1}\left(P_{E}-P_{P}\right)-A_{W 1}\left(P_{P}-P_{W}\right)+A_{E 2}\left(P_{N}+P_{N E}-P_{S}-P_{S E}\right)-A_{W 2}\left(P_{N}+P_{N W}-P_{S}-P_{S W}\right) \\
& +A_{N 1}\left(P_{N}-P_{P}\right)-A_{S 1}\left(P_{P}-P_{S}\right)+A_{N 2}\left(P_{E}+P_{N E}-P_{W}-P_{N W}\right) \\
& -A_{S 2}\left(P_{E}+P_{S E}-P_{W}-P_{S W}\right)=S_{P}
\end{aligned}
$$

with

$$
\begin{array}{ll}
A_{E 1}=\frac{2\left(H_{E}^{3} \phi_{x x E}\right)\left(H_{P}^{3} \phi_{x x P}\right)}{\left(H_{E}^{3} \phi_{x x E}+H_{P}^{3} \phi_{x x P}\right)} \frac{\Delta y}{\Delta x} & A_{W 1}=\frac{2\left(H_{W}^{3} \phi_{x x W}\right)\left(H_{P}^{3} \phi_{x x P}\right)}{\left(H_{W}^{3} \phi_{x x W}+H_{P}^{3} \phi_{x x P}\right)} \frac{\Delta y}{\Delta x} \\
A_{E 2}=\frac{\mathrm{K}}{4} \frac{2\left(H_{E}^{3} \phi_{x y E}\right)\left(H_{P}^{3} \phi_{x y P}\right)}{\left(H_{E}^{3} \phi_{x y E}+H_{P}^{3} \phi_{x y P}\right)} & A_{W 2}=\frac{\mathrm{K}}{4} \frac{2\left(H_{W}^{3} \phi_{x y W}\right)\left(H_{P}^{3} \phi_{x y P}\right)}{\left(H_{W}^{3} \phi_{x y}+H_{P}^{3} \phi_{x y P}\right)}
\end{array}
$$




$$
\begin{aligned}
& A_{N 1}=\mathrm{K}^{2} \frac{2\left(H_{N}^{3} \phi_{y y N}\right)\left(H_{P}^{3} \phi_{y y P}\right)}{\left(H_{N}^{3} \phi_{y y N}+H_{P}^{3} \phi_{y y P}\right)} \frac{\Delta x}{\Delta y} \quad A_{S 1}=\mathrm{K}^{2} \frac{2\left(H_{S}^{3} \phi_{y y S}\right)\left(H_{P}^{3} \phi_{y y P}\right)}{\left(H_{S}^{3} \phi_{y y S}+H_{P}^{3} \phi_{y y P}\right)} \frac{\Delta x}{\Delta y} \\
& A_{N 2}=\frac{\mathrm{K}}{4} \frac{2\left(H_{N}^{3} \phi_{y x N}\right)\left(H_{P}^{3} \phi_{y x P}\right)}{\left(H_{N}^{3} \phi_{y x N}+H_{P}^{3} \phi_{y x P}\right)} \quad A_{S 2}=\frac{\mathrm{K}}{4} \frac{2\left(H_{S}^{3} \phi_{y x S}\right)\left(H_{P}^{3} \phi_{y x P}\right)}{\left(H_{S}^{3} \phi_{y x S}+H_{P}^{3} \phi_{x y P}\right)} \\
& S_{P}=6 \zeta \Delta y\left[\left(\frac{2\left(H_{P}\right)\left(H_{E}\right)}{\left(H_{P}+H_{E}\right)}-\frac{2\left(H_{P}\right)\left(H_{W}\right)}{\left(H_{P}+H_{W}\right)}\right)+\left(\frac{\phi_{c . c W}-\phi_{c . c E}}{2}\right)\right] \\
& +6 \zeta \Delta y\left[\frac{\phi_{\text {s.c. } x E}-\phi_{\text {s.c. } . W}}{2}\right]+6 \zeta \mathrm{K} \Delta x\left[\frac{\phi_{\text {s.c. } . y N}-\phi_{s . c . y S}}{2}\right]
\end{aligned}
$$

Equation 4.9 can then be written as,

$$
A_{P} P_{P}=A_{E} P_{E}+A_{W} P_{W}+A_{N} P_{N}+A_{S} P_{S}+A_{N E} P_{N E}+A_{S E} P_{S E}+A_{N W} P_{N W}+A_{S W} P_{S W}-S_{P}
$$

with

$$
\begin{array}{lll}
A_{P}=A_{E 1}+A_{W 1}+A_{N 1}+A_{S 1} & A_{E}=A_{E 1}+A_{N 2}-A_{S 2} & A_{W}=A_{W 1}+A_{S 2}-A_{N 2} \\
A_{N}=A_{N 1}+A_{E 2}-A_{W 2} & A_{S}=A_{S 1}+A_{W 2}-A_{E 2} & A_{N E}=A_{E 2}+A_{N 2} \\
A_{S E}=-A_{E 2}-A_{S 2} & A_{N W}=-A_{W 2}-A_{N 2} & A_{S W}=A_{W 2}+A_{S 2}
\end{array}
$$

In this manner, it is possible to construct the required number of equations needed to solve the Reynolds equation. The solution for the equations is found using the alternating-direction implicit (ADI) method, with the tridiagonal matrix algorithm (TDMA) solving for the columns of the matrix, and the cyclic tridiagonal matrix algorithm (CTDMA) solving for the rows. 
The linear equations are solved in the y-direction for the columns using,

$$
A(I) P(I)=B(I) P(I+1)+C(I) P(I-1)+D(I)
$$

with:

$$
\begin{aligned}
& A(I)=A_{P}=A_{E 1}+A_{W 1}+A_{N 1}+A_{S 1} \\
& B(I)=A_{N}=A_{N 1}+A_{E 2}-A_{W 2} \\
& C(I)=A_{S}=A_{S 1}+A_{W 2}-A_{E 2} \\
& D(I)=A_{E} P_{E}+A_{W} P_{W}+A_{N E} P_{N E}+A_{S E} P_{S E}+A_{N W} P_{N W}+A_{S W} P_{S W}-S_{P}
\end{aligned}
$$

Temporary pressure values are then calculated after each run through with the TDMA subroutine and values for the $\phi$ 's are updated. Under-relaxation is applied as new and old pressure values are continuously compared, until the desired tolerance is met.

The linear equations are solved in the $\mathrm{x}$-direction for the rows using,

$$
A(J) P(J)=B(J) P(J+1)+C(J) P(J-1)+D(J)
$$

with:

$$
\begin{aligned}
& A(J)=A_{P}=A_{E 1}+A_{W 1}+A_{N 1}+A_{S 1} \\
& B(J)=A_{E}=A_{E 1}+A_{N 2}-A_{S 2} \\
& C(J)=A_{W}=A_{W 1}+A_{S 2}-A_{N 2} \\
& D(J)=A_{N} P_{N}+A_{S} P_{S}+A_{N E} P_{N E}+A_{S E} P_{S E}+A_{N W} P_{N W}+A_{S W} P_{S W}-S_{P}
\end{aligned}
$$

As with the columns, temporary values for pressure in the rows are then calculated after each run through with the CTDMA subroutine and the $\phi$ 's are updated. 
Under-relaxation is applied as new and old pressure values are continuously compared until the desired tolerance is met and convergence occurs.

\subsection{Computational Procedure}

The computational procedure for the full elastohydrodynamic analysis is shown in Figure 4.2 and consists of two nested iteration loops. This is an expanded version of the scheme used in the preliminary hydrodynamic study. In the hydrodynamic analysis the deformation properties are entered as constant values, therefore only one iteration loop was needed to solve for the pressure distribution in the fluid. The procedure in the figure is the expanded and final scheme used for the elastohydrodynamic analysis and requires two iteration loops. In the inner loop, the finite difference equations are employed and a solution for the pressure in the lubricating film is found using initial guesses for $\delta, \mathrm{H}, \gamma$, $\Psi$ and $\theta$. In the outer loop Equations 3.18, 3.32 and 3.34 are introduced and the acquired pressure distribution is used to define $\Psi, \mathrm{H}$ and $\delta$, and the procedure for calculating $\gamma$ and $\theta$ is introduced (Section 3.5). The change in $\mathrm{H}$ is modified with an under-relaxation factor and a test criterion for convergence is set. The current information enters the inner loop and a new solution for the pressure in the lubricating film is found. The outer loop is re-entered for the updating of $\mathrm{H}, \delta, \gamma, \Psi$ and $\theta$, and the relaxation and testing of $\mathrm{H}$. The iterative process continues going back and forth between the inner and outer loops as a final solution for the Reynolds equation and the deformation equations is reached for both the pressure and the average film thickness. 


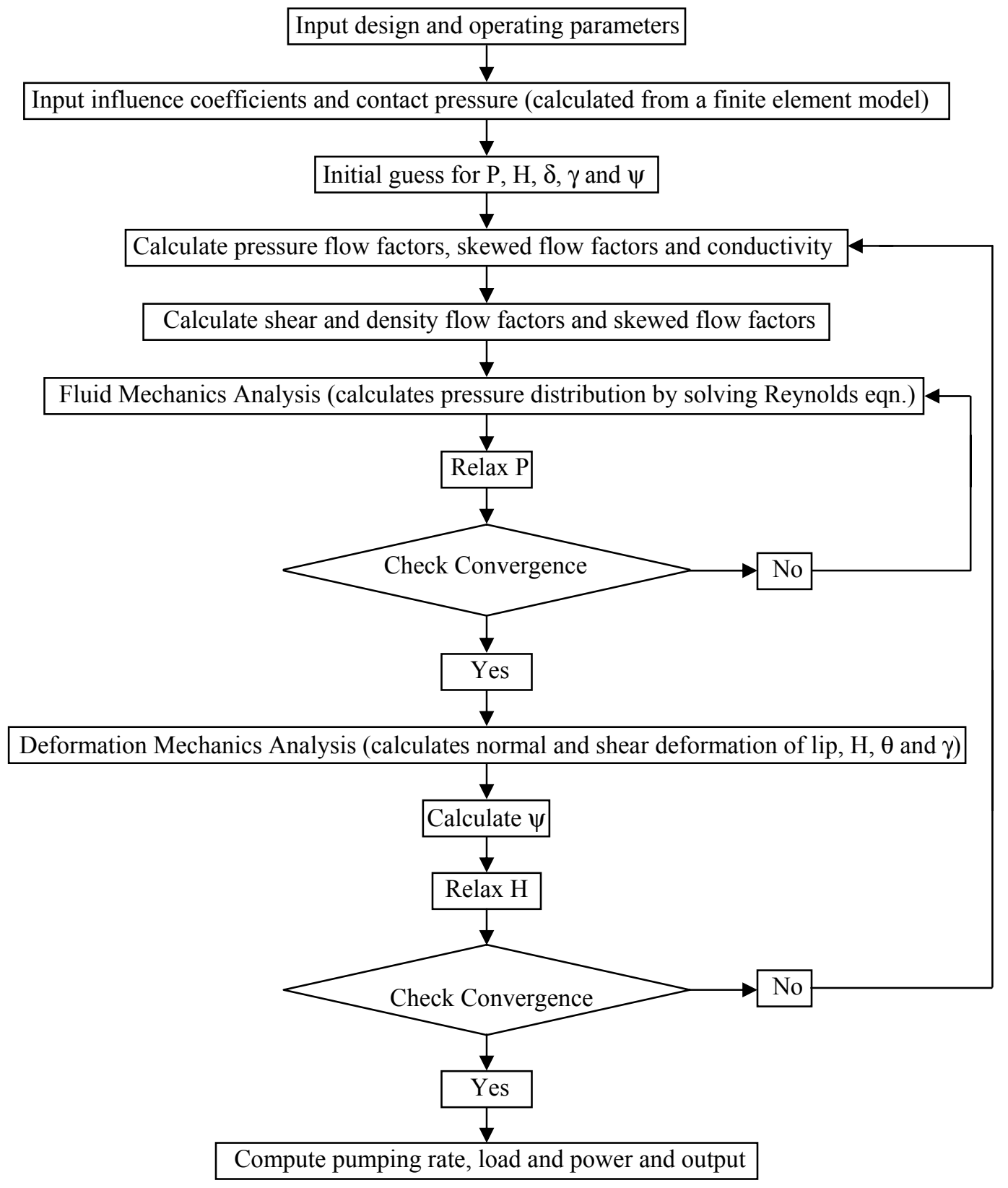

Figure 4.2 Computational Scheme 
Once the pressure distribution is computed, the load support is obtained by integrating the pressure over the solution space,

$\hat{W}=\int_{0}^{1} \int_{0}^{1} P d \hat{x} d \hat{y}$

The pumping rate is obtained from,

$\hat{Q}_{y}=\int_{0}^{1} \hat{q}_{y} d \hat{x}$

with,

$\hat{q}_{y}=-H^{3}\left(\phi_{y x} \frac{\partial P}{\partial x}+\mathrm{K} \phi_{y y} \frac{\partial P}{\partial y}\right)+6 \zeta \phi_{s . c . y}$

The power consumption is found with,

$\hat{\Pi}=\left[\int_{0}^{1} \hat{\tau}_{\text {avg }} d \hat{y}\right] \hat{R} \zeta$

with,

$\hat{R}=\frac{R}{L_{y}}$

An $81 \times 81$ mesh is used (selected following a mesh sensitivity study), and the program is written in FORTRAN. A relaxation factor of 0.1 and an allowable convergence error of $10^{-5}$ are used. 


\section{CHAPTER V}

\section{RESULTS}

\subsection{Preliminary Hydrodynamic Analysis}

For this phase of the study (Salant and Rocke, 2004), film thickness and asperity aspect ratio and orientation angle are assigned constant values and just the flow field of the lubricating film is analyzed. Computations are performed for a representative seal with dimensional base parameters of $: \mathrm{h}=2 \mu \mathrm{m}, \mathrm{L}_{\mathrm{x}}=\mathrm{L}_{\mathrm{y}}=0.1 \mathrm{~mm}, \sigma=1 \mu \mathrm{m}, \lambda_{\mathrm{y}}=5 \mu \mathrm{m}$, $\lambda_{\mathrm{x}}=1.667 \mu \mathrm{m}$ and $0.556 \mu \mathrm{m}, \mathrm{y}_{\mathrm{b}}=0.07 \mathrm{~mm}, \mathrm{p}_{\mathrm{s}}=\mathrm{p}_{\mathrm{a}}=10^{5} \mathrm{~Pa}, \mathrm{p}_{\mathrm{c}}=0 \mathrm{~Pa}, \mu=0.025 \mathrm{~Pa}-\mathrm{s}, \mathrm{U}$ $=8 \mathrm{~m} / \mathrm{s}$, and dimensionless base parameters of: $\mathrm{H}=2, \mathrm{~K}=1, \gamma=3$ and $9, \hat{y}_{b}=0.7, \mathrm{p}_{\mathrm{s}}=1$, $\alpha=0.001, \zeta=200$. Using a $2 \mathrm{GHz}$ PC, the base case requires a computation time of approximately 15 minutes. The computation time for a comparable deterministic solution is estimated at approximately 90 minutes.

The pressure distribution in the lubricating film for the base case is shown in Figure 5.1. It is apparent that the distorted asperity distribution together with the motion of the shaft produces elevated pressures sufficient to keep the lip lifted off the shaft. The maximum pressure occurs at $\hat{y}_{b}=0.7$, the location of the maximum circumferential displacement. This is an example of a successful seal in which the maximum circumferential displacement is located at a point closer to the liquid-side of the seal than to the air-side.

Figure 5.2 shows an example of the pressure distribution when the maximum circumferential displacement is closer to the air-side of the seal $\left(\hat{y}_{b}=0.3\right)$. It is shown in 
Figure 5.10 that although this configuration produces load support, it results in pumping the fluid toward the air-side of the seal, thus promoting leakage.

These figures also show that the asperities with the larger aspect ratio of $\gamma=9$ produce higher elevated pressures and more load support than the asperities with the smaller aspect ratio of $\gamma=3$.

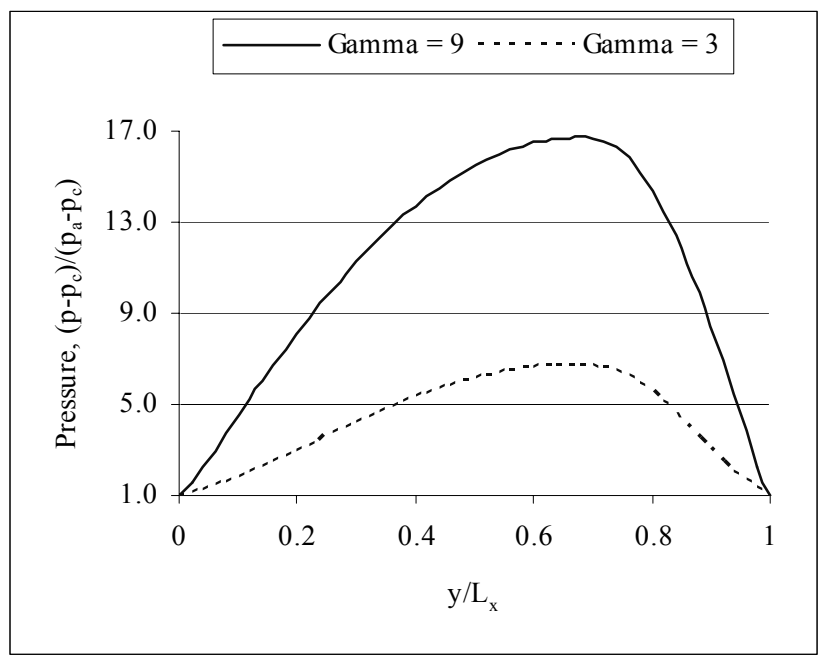

Figure 5.1 Pressure Distribution, Base Case, $\hat{y}_{b}=0.7$

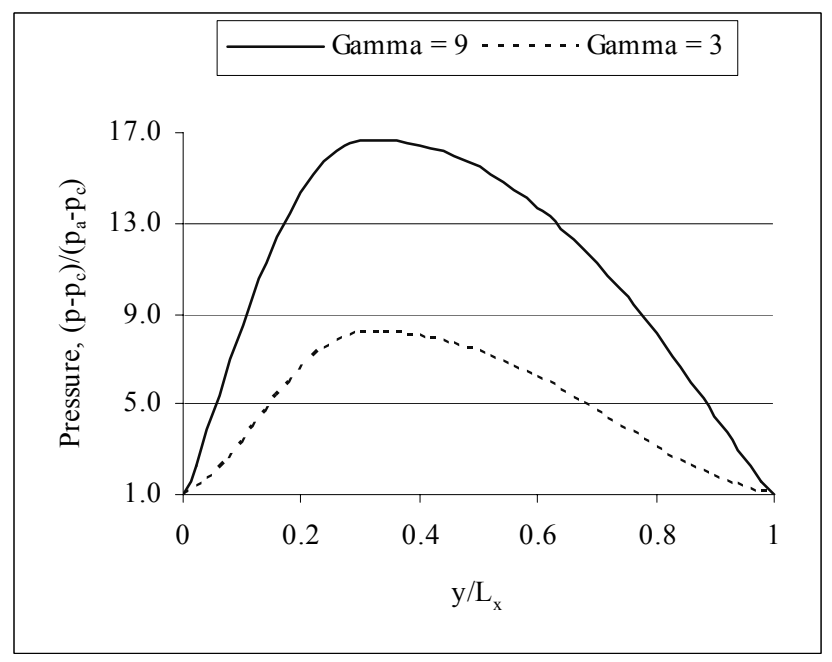

Figure 5.2 Pressure Distribution, $\hat{y}_{b}=0.3$ 
Figures 5.3 and 5.4 show the effect that shaft speed has on the load support and pumping rate. As expected, more load support is produced with increasing shaft speed. The pumping rate is affected similarly as the seal behavior is like that of a viscous pump, with the more vane-like asperity aspect ratio of $\gamma=9$ producing the greater effect. In both cases, the slope of the curve decreases at higher speeds for $\gamma=3$. This also occurs for $\gamma=9$ at speeds beyond the range of these graphs due to the increasing influence of cavitation.

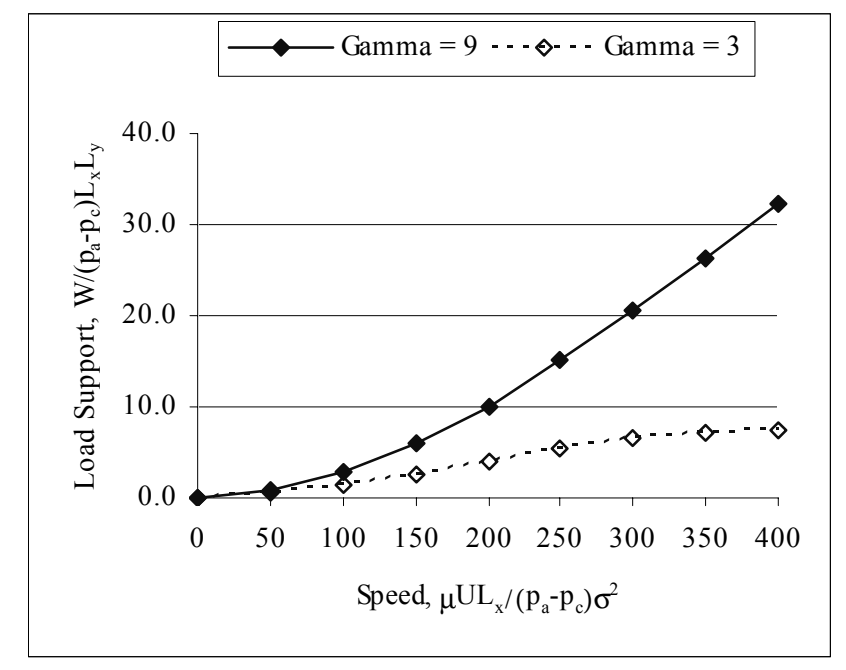

Figure 5.3 Load Support vs. Speed 


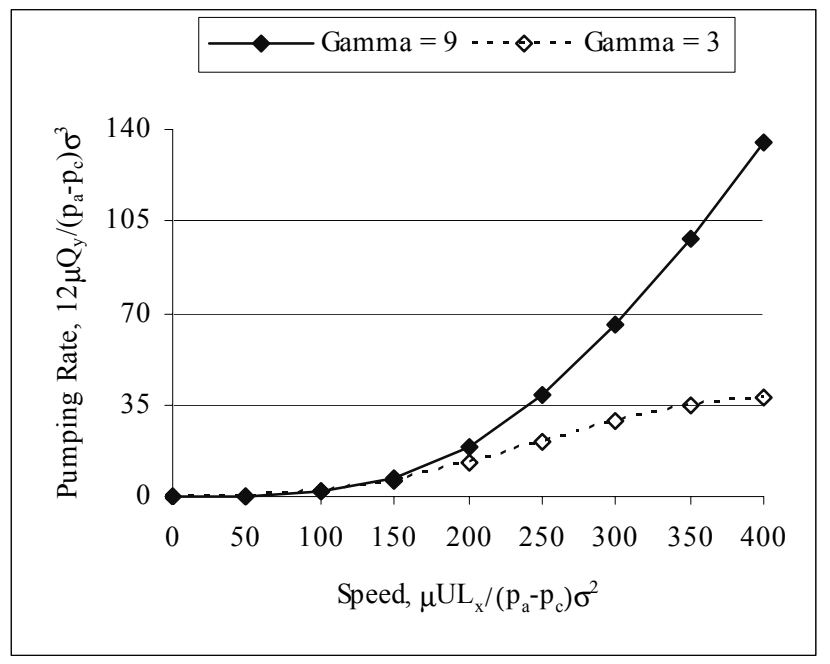

Figure 5.4 Pumping Rate vs. Speed

The effect of increasing film thickness on load support is shown in Figure 5.5. As the film thickness rises, the load support decreases. This makes sense because the asperity heights decrease relative to the film thickness.

The pumping rate responds differently as the film thickness is increased, as shown in Figure 5.6. Initially, the flow area is increased causing the pumping rate to increase. However, at some point the film reaches a thickness that lessens the asperities effect on the pumping rate, which in turn begins to decrease. 


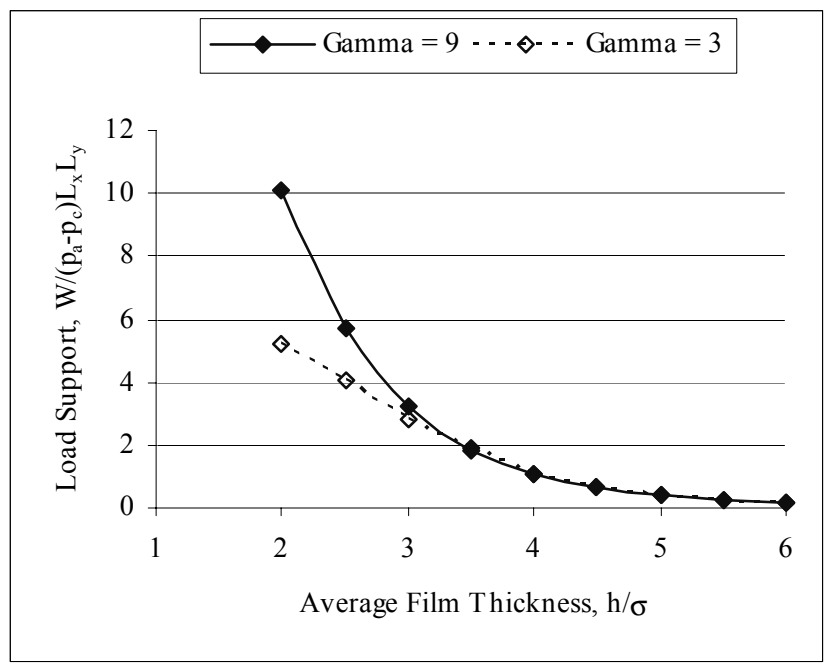

Figure 5.5 Load Support vs. Average Film Thickness

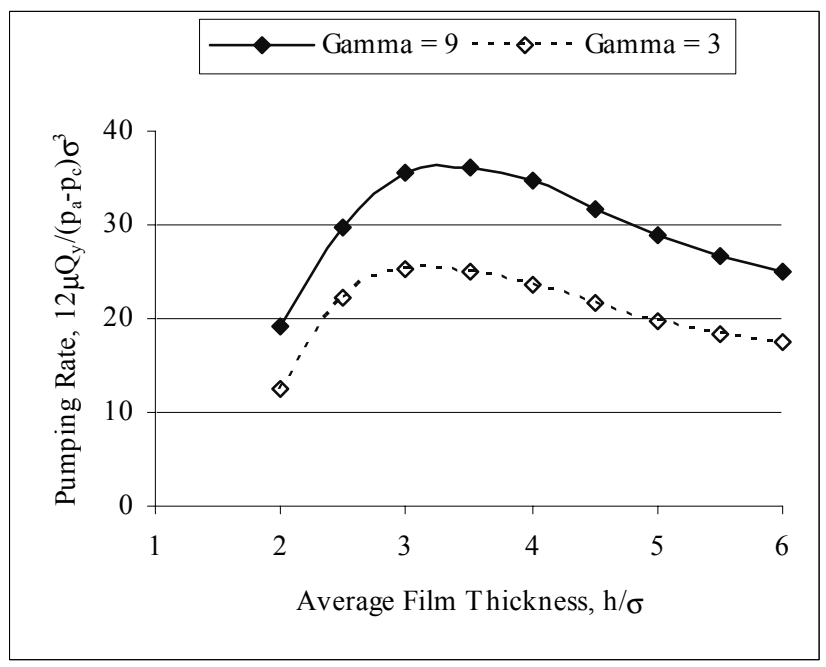

Figure 5.6 Pumping Rate vs. Average Film Thickness

The lip's ability to deform is represented by the elasticity parameter $\alpha$. A larger value of $\alpha$ represents a more elastic lip that results in a larger lip deformation. Figures 5.7 and 5.8 show how an increasing circumferential deformation affects the load support 
and pumping rate. In both cases there is an increase until a certain value is reached and then a decrease (for $\gamma=9$ this happens outside of the range of the graph in Figure 5.8). This result makes sense because an increase in the elasticity changes the distribution of the asperity orientation angle $\theta$. The maximum values in the figures would indicate the point at which an optimum vane angle is reached, resulting in the most effective viscous pumping action.

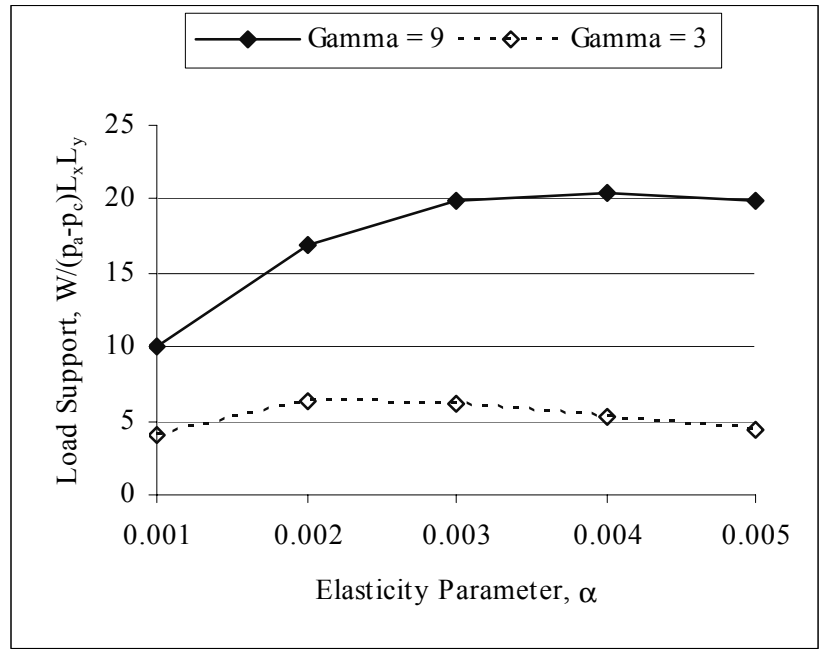

Figure 5.7 Load Support vs. Elasticity Parameter 


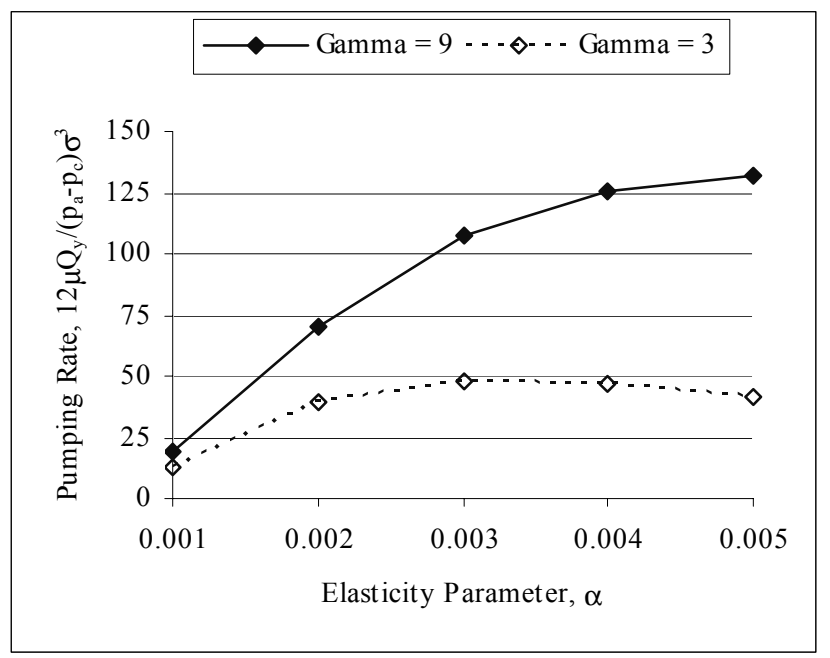

Figure 5.8 Pumping Rate vs. Elasticity Parameter

The axial location $\left(\hat{y}_{b}\right)$ of the maximum circumferential displacement of the lip surface is critical to sealing ability. Figure 5.9 shows that a maximum load support is produced at $\hat{y}_{b}=0.5$, with symmetry about that point. Figure 5.10 shows that the pumping rate will be zero at this point, and that negative pumping will occur at lower values of $y$ and positive pumping will occur at higher values of $y$. This explains why the maximum circumferential displacement of the lip must be located at a point closer to the liquid-side of the seal than to the air-side $\left(\hat{y}_{b}>0.5\right)$. 


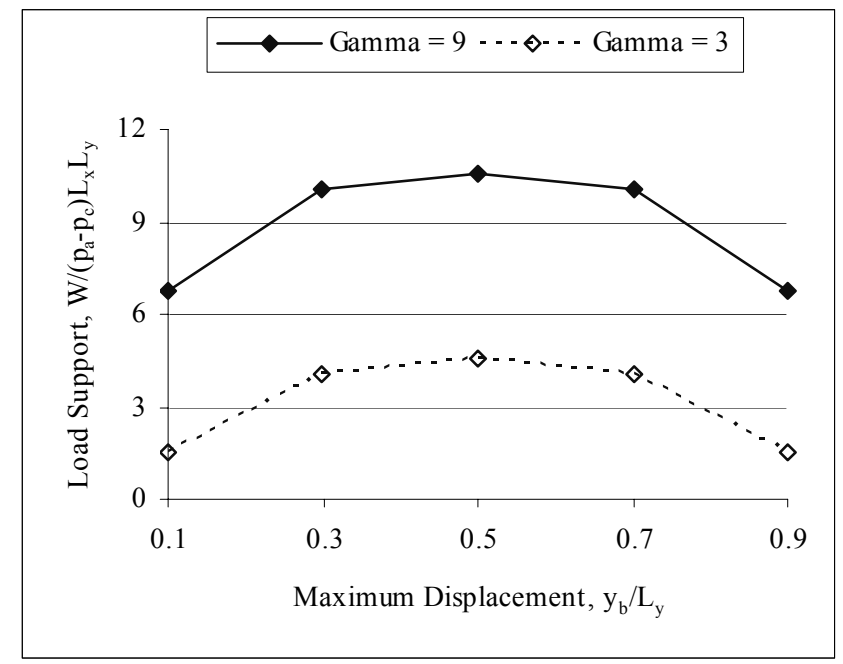

Figure 5.9 Load Support vs. $\hat{y}_{b}$

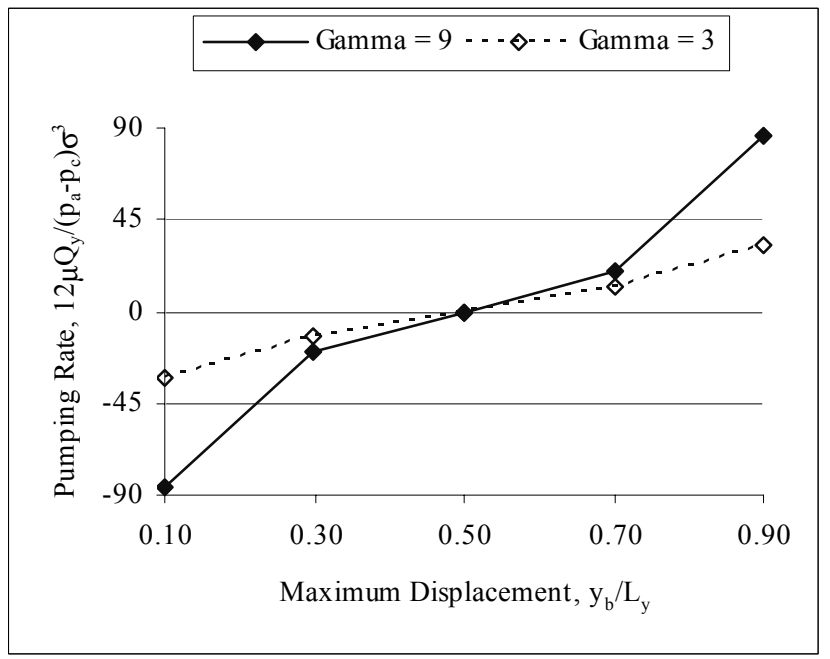

Figure 5.10 Pumping Rate vs. $\hat{y}_{b}$

With the exception of Figures 5.11 and 5.12, results for this analysis have been based on the boundary conditions used by manufacturers to measure pumping rate $\left(\mathrm{y}_{0}=\right.$ $\mathrm{y}_{1}=$ ambient pressure). The sealed pressure is varied in these figures to show the effect 
on load support and pumping rate. Figure 5.11 shows a negligible effect on load support for the range of sealed pressure tested and Figure 5.12 shows the expected decrease in pumping rate due to the increased pressure difference at the liquid-side of the seal.

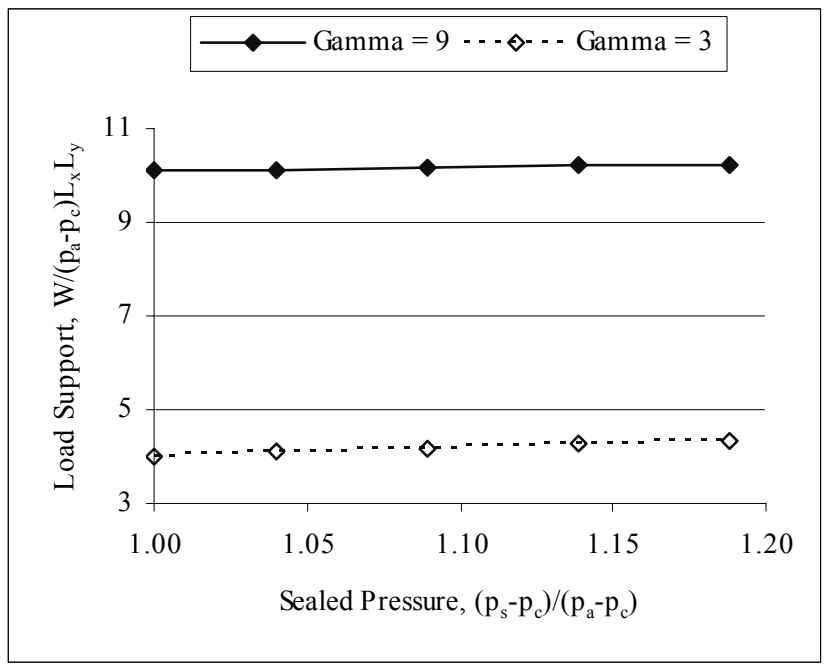

Figure 5.11 Load Support vs. Sealed Pressure

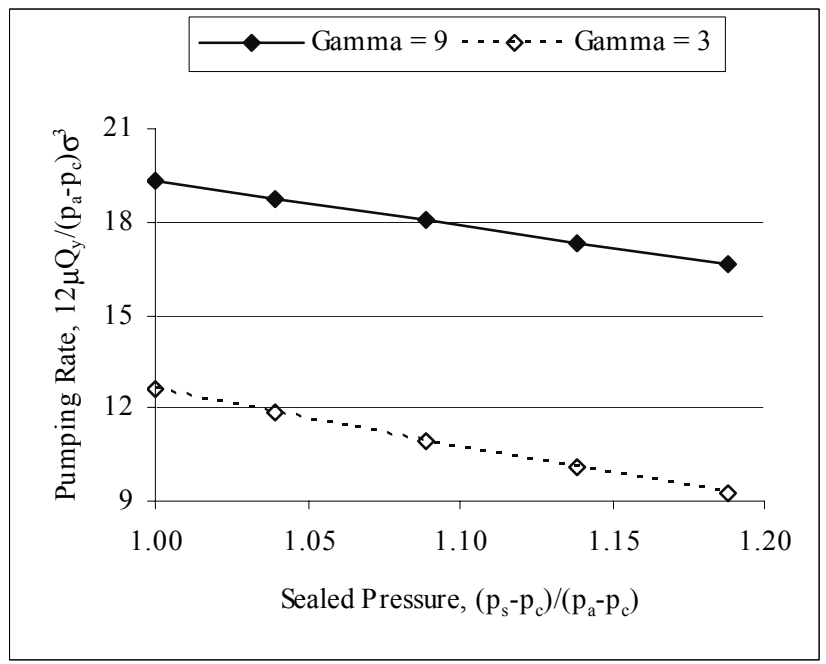

Figure 5.12 Pumping Rate vs. Sealed Pressure 


\subsection{Implementing the Normal Deformation Calculation}

The program was upgraded gradually to facilitate the integration of the various parts of the model. In this second phase, the original assumed displacement pattern for the asperities and constant aspect ratio values were left unchanged, while the normal deformation and film thickness were computed. The normal coefficient matrix and contact pressure values from the finite element analysis performed by Shen (2002) was used for the analysis. At the same time, the validity of the extrapolation algorithm for $1.00 \leq \mathrm{H}<2.00$ was tested.

Tests were run for a representative seal with dimensional base parameters of: $L_{x}$ $=\mathrm{L}_{\mathrm{y}}=0.1 \mathrm{~mm}, \sigma=1 \mu \mathrm{m}, \mathrm{A}_{1}=1.4 \mu \mathrm{m}, \lambda_{\mathrm{y}}=5 \mu \mathrm{m}, \lambda_{\mathrm{x}}=0.556 \mu \mathrm{m}, \mathrm{y}_{\mathrm{b}}=0.07 \mathrm{~mm}, \mathrm{p}_{\mathrm{s}}=\mathrm{p}_{\mathrm{a}}$ $=10^{5} \mathrm{~Pa}, \mathrm{p}_{\mathrm{c}}=0 \mathrm{~Pa}, \mu=0.025 \mathrm{~Pa}-\mathrm{s}, \mathrm{U}=8 \mathrm{~m} / \mathrm{s}, \mathrm{E}=6.2 \times 10^{6} \mathrm{~Pa}$, and dimensionless base parameters of : $\hat{A}_{1}=1.4, \mathrm{~K}=1, \gamma=9, \mathrm{y}_{\mathrm{b}}=0.7, \mathrm{p}_{\mathrm{s}}=1, \alpha=0.001, \zeta=200$.

Figure 5.13 shows that the converged values for $\mathrm{H}$ in the axial direction vary slightly, (from 1.732 to 1.744 ). This is consistent with previous studies in which it has been shown that the average film thickness (averaged circumferentially) is relatively constant across the sealing zone. 


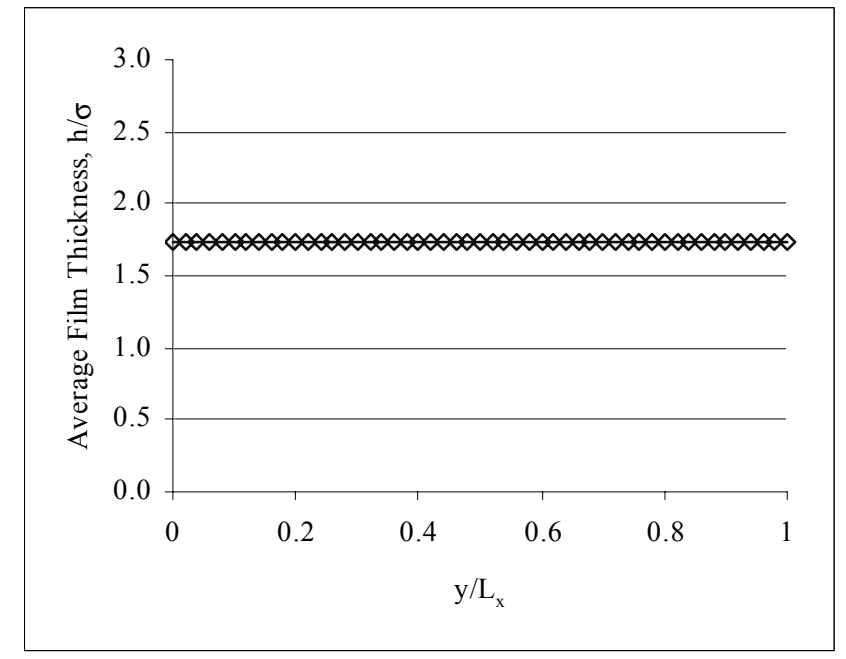

Figure 5.13 Calculated Average Film Thickness Distribution

Figures 5.14 and 5.15 show comparisons of the pressure distribution in the film for solutions to the Reynolds equation using a constant $\mathrm{H}=2.00$ before the addition of the normal deformation analysis, and one in which the film thickness is calculated and the extrapolation program for $\mathrm{H}<2.00$ is utilized. The magnitude of the pressure in the latter is increased, as would be expected since the computed film thickness of the latter is lower than that set in the former. Also, the maximum pressure occurs at the axial location of maximum displacement $\left(y_{b}=0.7\right)$ in both solutions, as expected. 


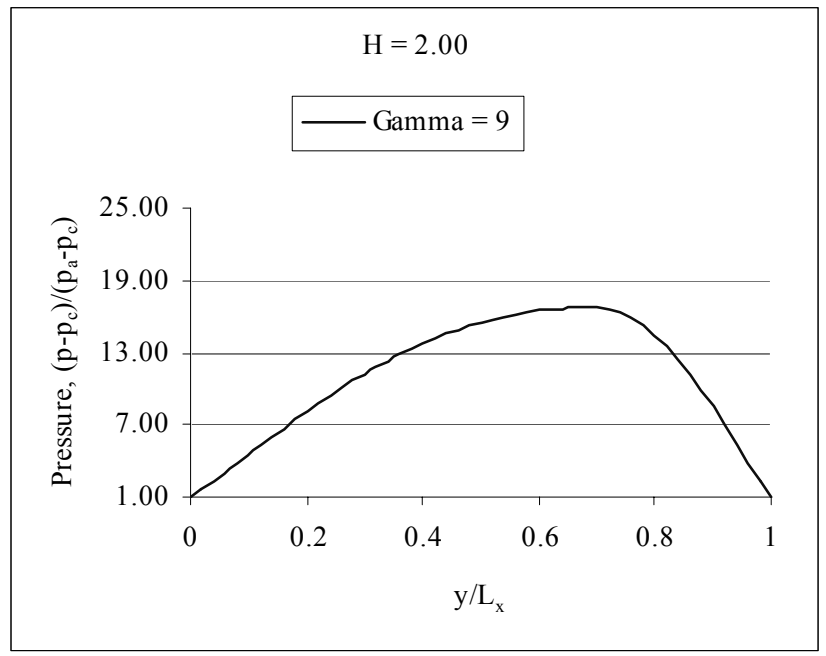

Figure 5.14 Pressure Distribution for $\mathrm{H}=2.00$ before Calculation of Normal Deformation

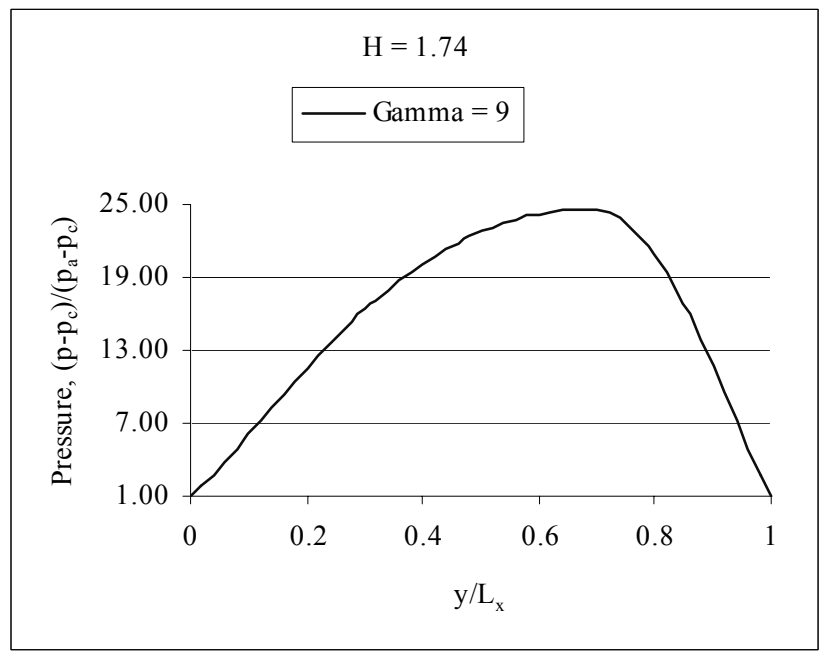

Figure 5.15 Pressure distribution for $\mathrm{H}=1.74$ using Extrapolation \& Normal Deformation Analysis 
Figures 5.16 and 5.17 show the variation in pumping rate and load support with average film thickness, for cases in which the film thickness is computed (not set). The film thickness is varied by varying the value of $\hat{A}_{1}$, the static undeformed film thickness. These graphs show the additional solutions for $\mathrm{H}$ when $\hat{A}_{1}=0.7,0.9$ and $1.4(\mathrm{H}<2.00)$. The results show that the behavior of the pumping rate and load as $\mathrm{H}$ is decreased below values of 2.00 is consistent with previous tests performed using $2.00 \leq \mathrm{H} \leq 6.00$.

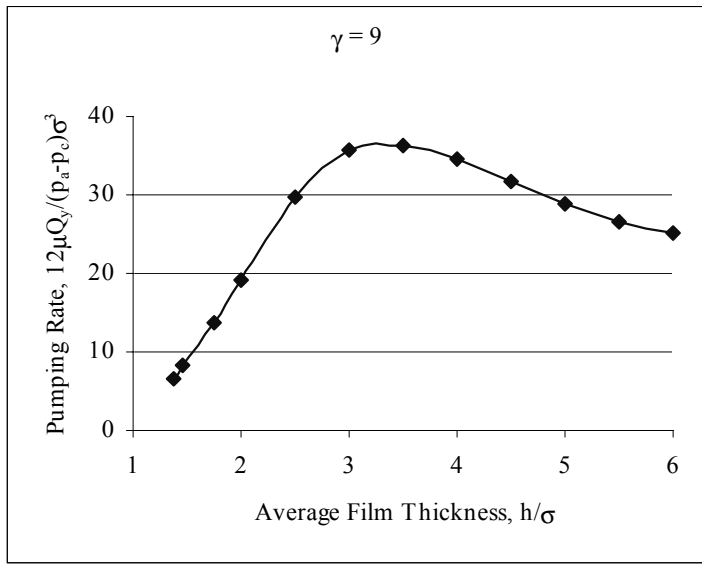

Figure 5.16 Pumping rate vs. Average Film Thickness

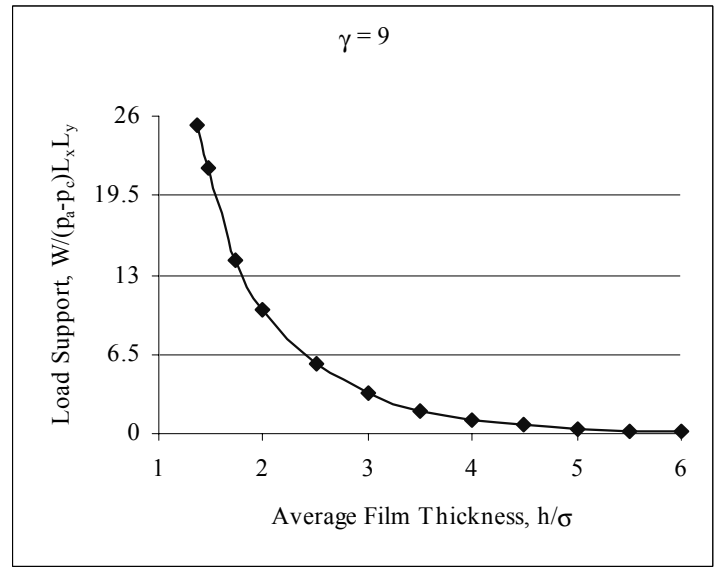

Figure 5.17 Load support vs. Average Film Thickness

The results of the second phase of the analysis indicate that the implementation of the extrapolation program which provides for the addition of shear flow factor calculations for $1.00 \leq \mathrm{H}<2.00$, and the normal deformation analysis that calculates the film thickness are valid. 


\subsection{Full Elastohydrodynamic Analysis}

With the addition of the finite element analysis conducted for this work, a full elastohydrodynamic analysis could be performed. The following test results are for the seal described in Section 3.4 with dimensional base parameters of : $\mathrm{L}_{\mathrm{x}}=\mathrm{L}_{\mathrm{y}}=0.1 \mathrm{~mm}$, $\sigma=1 \mu \mathrm{m}, \lambda_{\mathrm{y}}=5 \mu \mathrm{m}, \lambda_{\mathrm{x}}=1.667 \mu \mathrm{m}$ and $0.556 \mu \mathrm{m}, \mathrm{p}_{\mathrm{s}}=\mathrm{p}_{\mathrm{a}}=1.02 \times 10^{5} \mathrm{~Pa}, \mathrm{p}_{\mathrm{c}}=0 \mathrm{~Pa}, \mu=$ $0.02 \mathrm{~Pa}-\mathrm{s}, \mathrm{U}=5 \mathrm{~m} / \mathrm{s}, \mathrm{E}=3 \times 10^{6} \mathrm{~Pa}, \mathrm{~A}_{1}=1.4 \times 10^{-6} \mathrm{~m}$, and dimensionless base parameters of: $\mathrm{K}=1, \hat{p}_{s}=\hat{p}_{a}=1, \zeta=100, \hat{R}=191.9, \hat{A}_{1}=1.4, \mathrm{~V}_{\mathrm{r}}=1$. Using a $2 \mathrm{GHz}$ PC, the base case requires a computation time of approximately 15 minutes. The computation time for a comparable deterministic solution is estimated at approximately 78 hours. The effect of changing the undeformed value of $\lambda_{y}$ (but keeping the undeformed value of $\gamma$ constant) was explored, but proved to have virtually no influence on the results.

Figure 5.18 shows a comparison between the contact pressure distribution from the finite element analysis and the pressure distribution in the lubricating film for the base case. It is seen that the distributions are very similar upon lift-off. The axial locations of the maximum pressure are almost the same and are closer to the oil-side than to the airside of the seal. This indicates that a distorted asperity pattern was produced with the maximum circumferential displacement at a location closer to the liquid-side of the seal. 


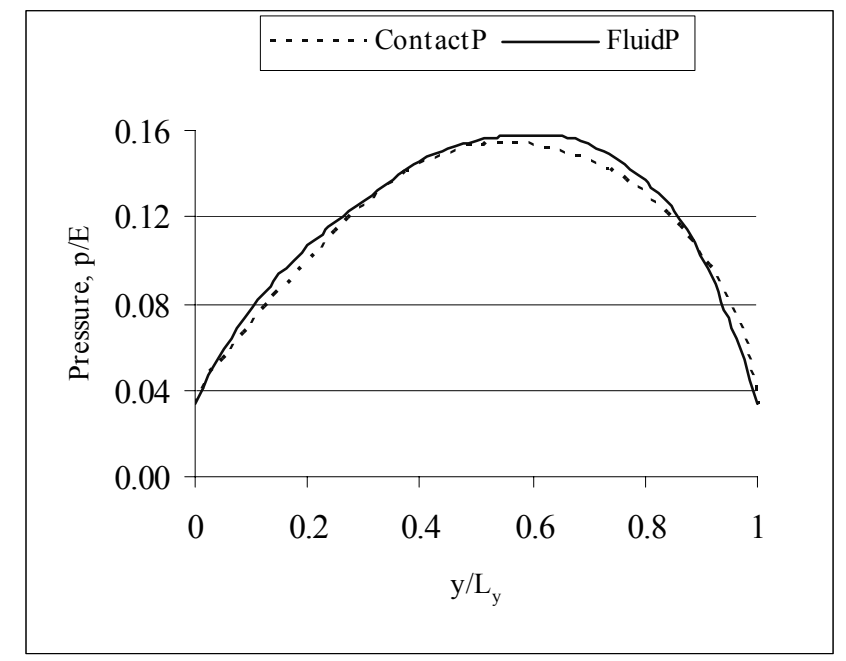

Figure 5.18 Contact Pressure and Fluid Pressure Distributions

As the shaft speed is increased, holding all other dimensional base parameters constant, the pressures increase slightly, producing larger normal deformations in the lip surface. This effect on average film thickness is shown in Figure 5.19. Upon lift-off the film is more uniform, and then becomes thicker and less uniform as the shaft speed increases. Lift-off is defined as the state when the fluid pressure overtakes the contact (pre-load) pressure.

Figure 5.20 shows the variation of shear deformation as it varies axially. The maximum location of deformation occurs closer to the liquid-side of the sealing zone, which is necessary for the reverse pumping action to occur. The shear deformations in this figure are of the same order and similar in shape to those measured in previous studies (Müller, 1987; Salant and Flaherty, 1994). 


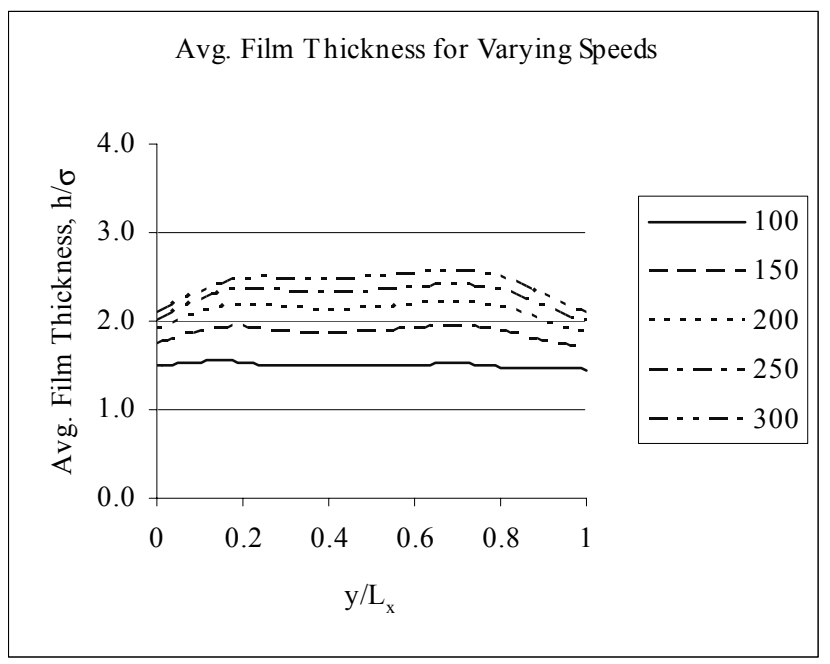

Figure 5.19 The Effect of Shaft Speed on Avg. Film Thickness

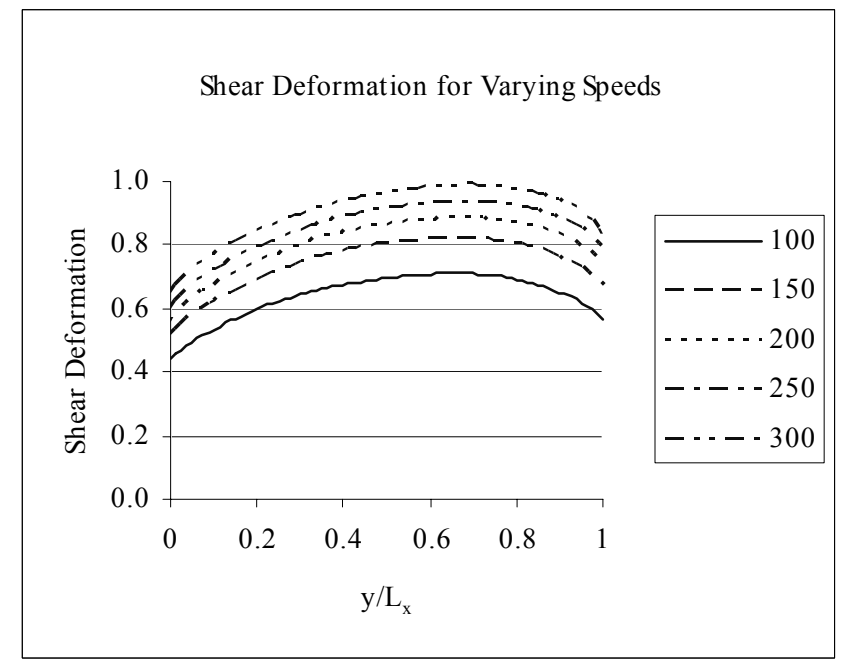

Figure 5.20 The Effect of Shaft Speed on Shear Deformation

Figures 5.21 and 5.22 show the effect that shaft speed has on the load support and pumping rate. As in the results from the hydrodynamic analysis, more load support is produced with increasing shaft speed. The pumping rate also rises as the increased shear 
deformation of the lip surface and resulting distorted asperity distribution act like a viscous pump.

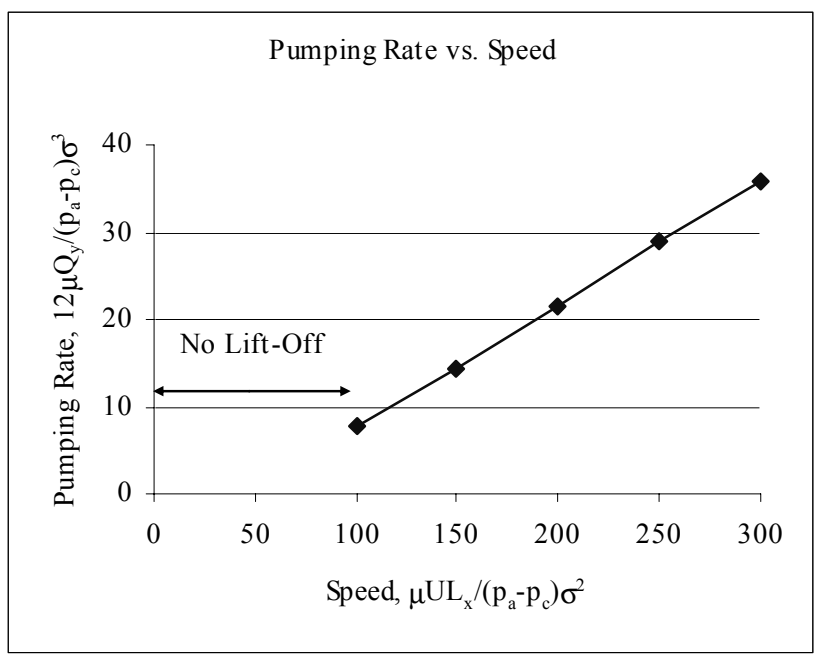

\subsection{Pumping Rate vs. Speed}

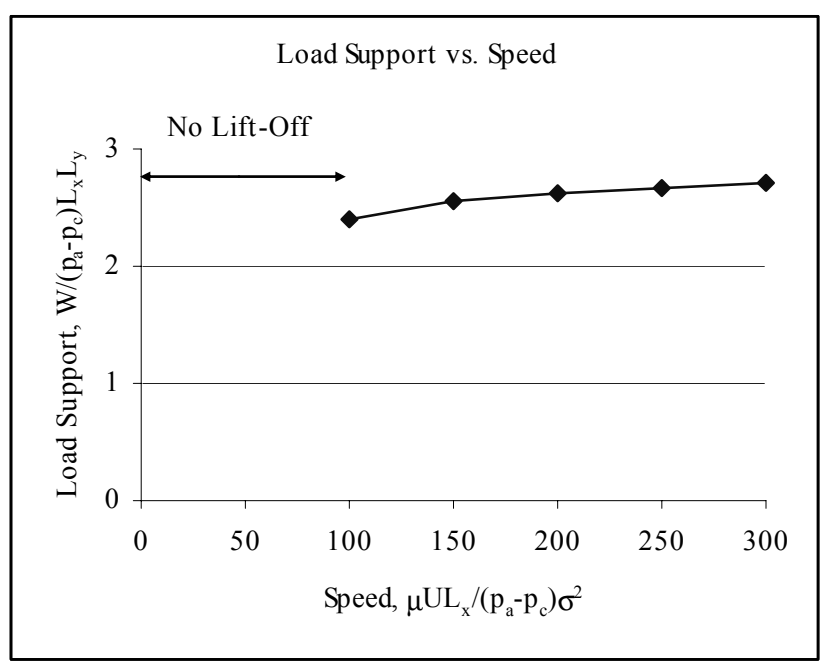

5.22 Load Support vs. Speed 
Figure 5.23 shows that the amount of power consumed rises continuously as the shaft speed increases. This is expected since the power consumption is directly proportional to the velocity.

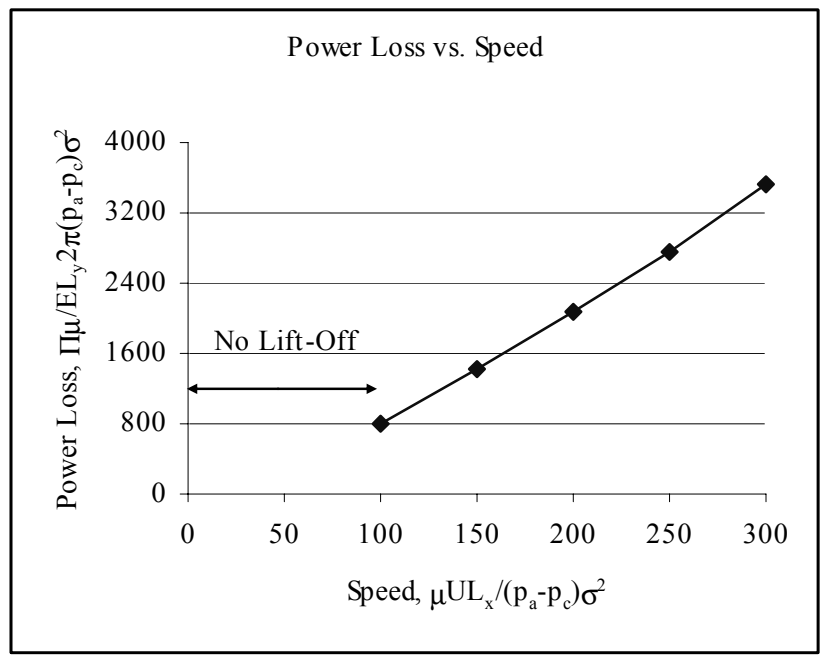

\subsection{Power Loss vs. Speed}

The results in Figure 5.24 show again the effect of speed on the average film thickness. This plot shows more clearly that the rate of increase is higher initially and then it begins to level off. As mentioned earlier, after lift-off occurs the pressures in the film will increase with speed, but only slightly. 


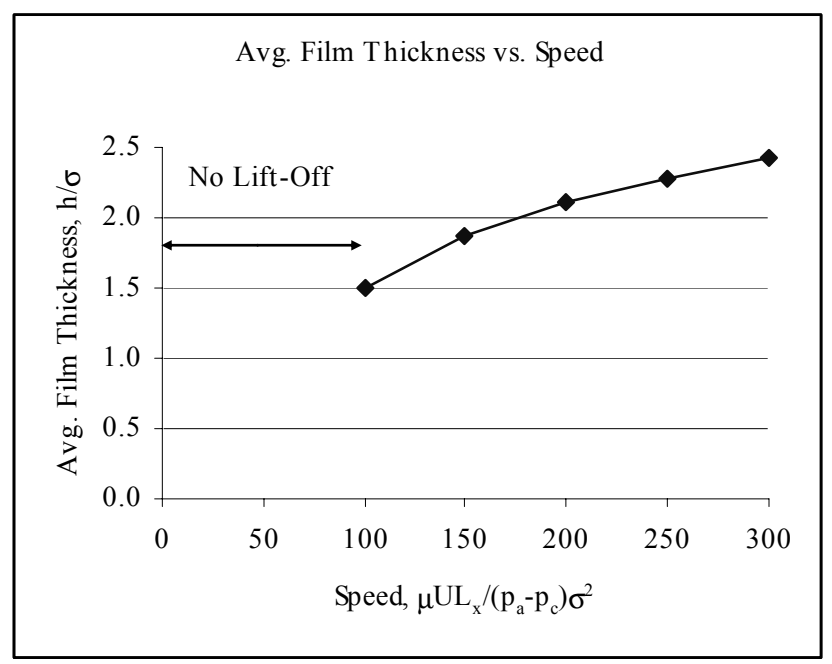

5.24 Average Film Thickness vs. Speed

Figure 5.25 shows that the maximum shear deformations in the rubber surface increase with increasing speed as expected.

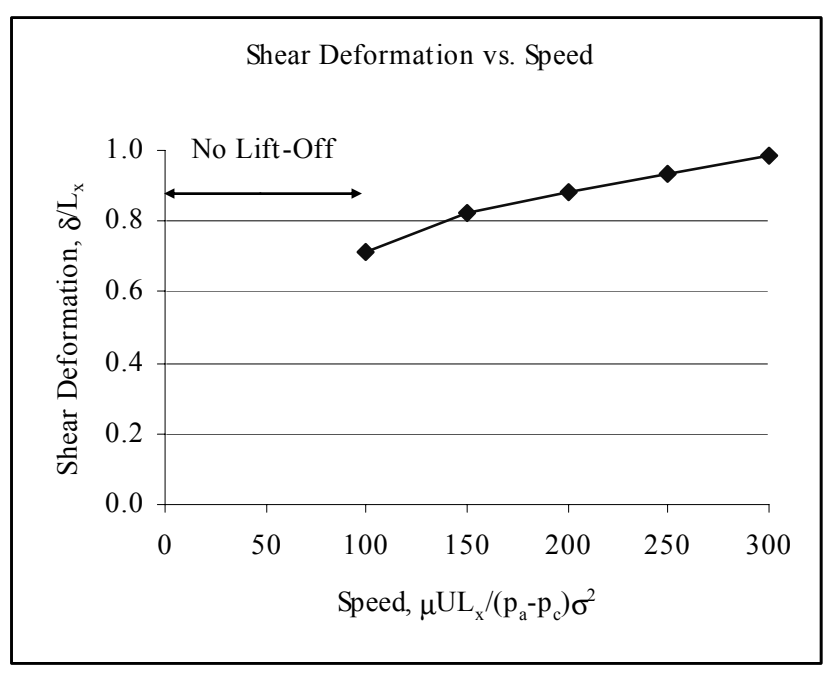

5.25 Maximum Shear Deformation vs. Speed 
The effect of viscosity, holding all other dimensional base parameters constant, on the pumping rate is shown in Figure 5.26. An increased pumping rate is expected because as the viscosity increases, the shear stresses within the fluid increase which will result in a higher pumping rate.

Figure 5.27 shows a steady increase in load with increased viscosity after lift-off occurs. This is expected because the increasing pumping rates create higher pressure elevations.

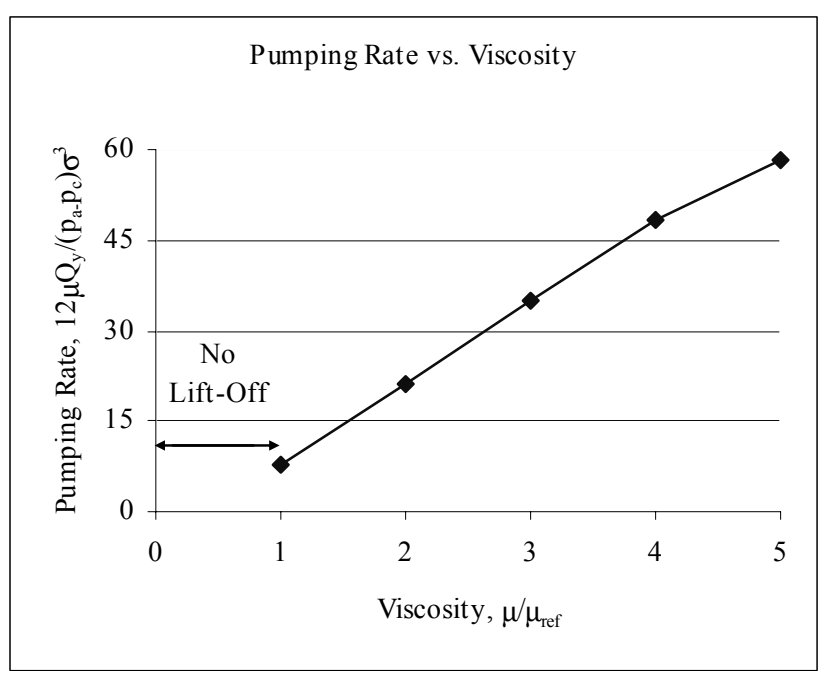

5.26 Pumping Rate vs. Viscosity 


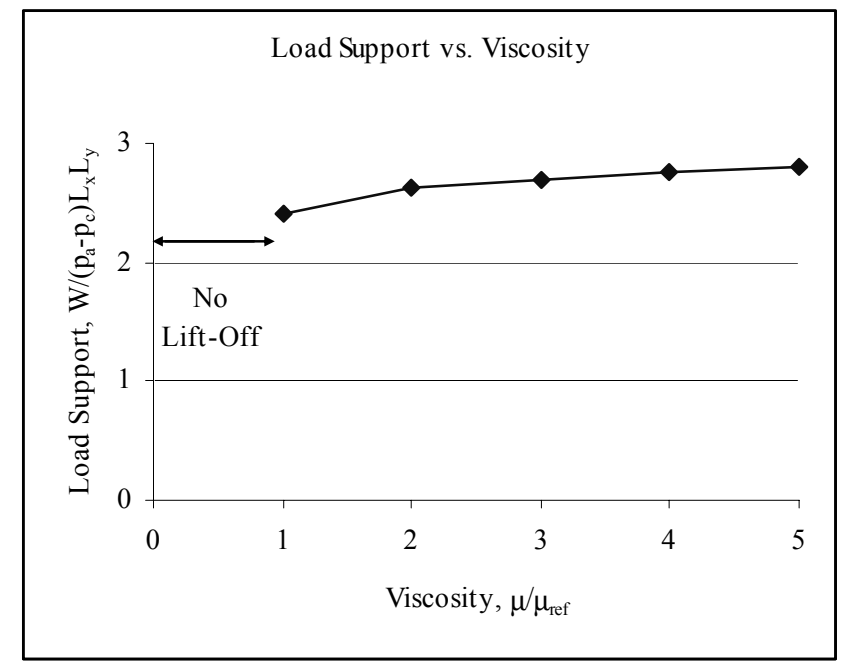

5.27 Load Support vs. Viscosity

The effect of higher viscosities is shown again in Figure 5.28 as it pertains to power consumption. A fluid with a higher viscosity contains higher values of shear stress, requiring more power to be consumed.

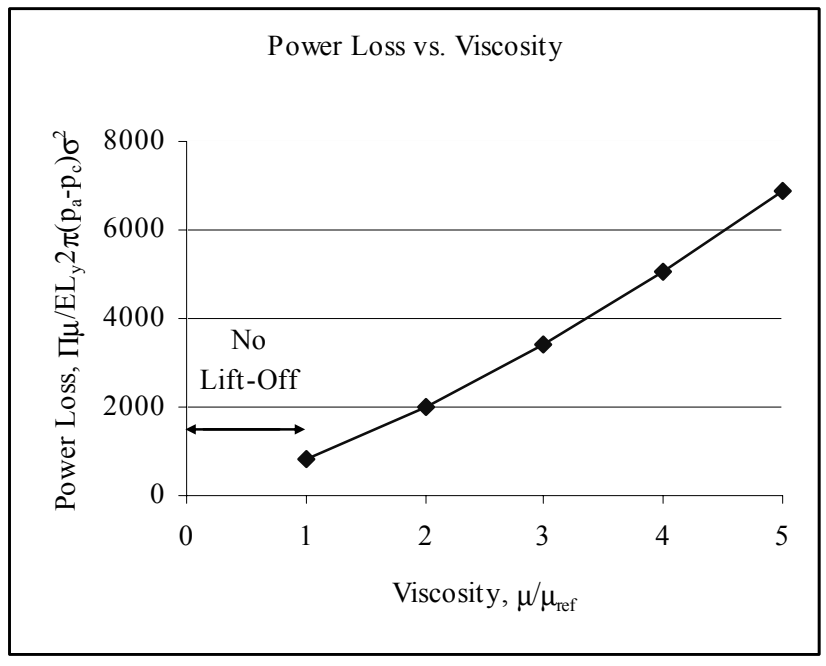

5.28 Power Loss vs. Viscosity 
Figure 5.29 shows that the average film thickness increases with higher viscosities, as expected, due to the increased load support and pumping rates.

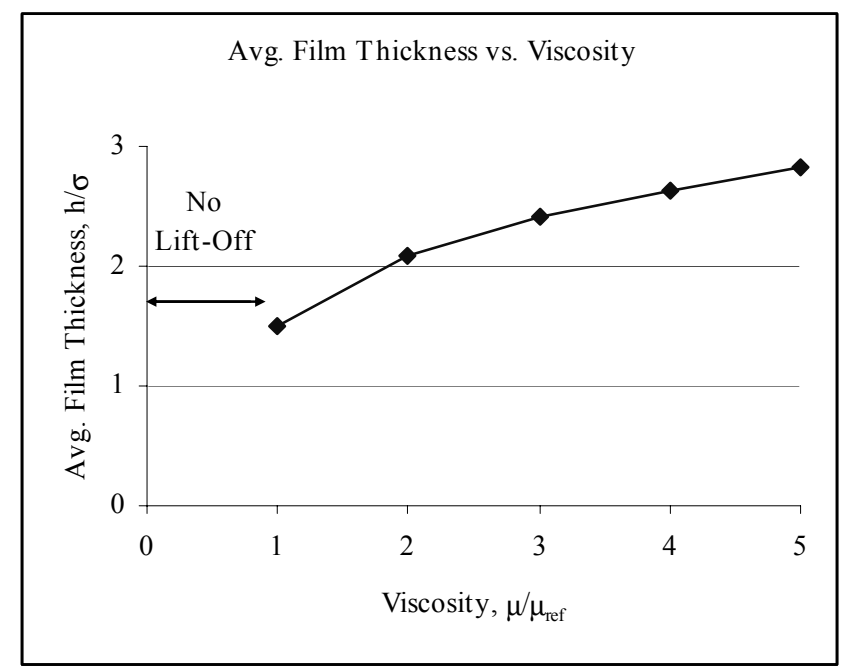

5.29 Average Film Thickness vs. Viscosity 
The maximum shear deformation is a function of viscous shear stress and is shown in Figure 5.30 to increase at higher viscosities, as expected.

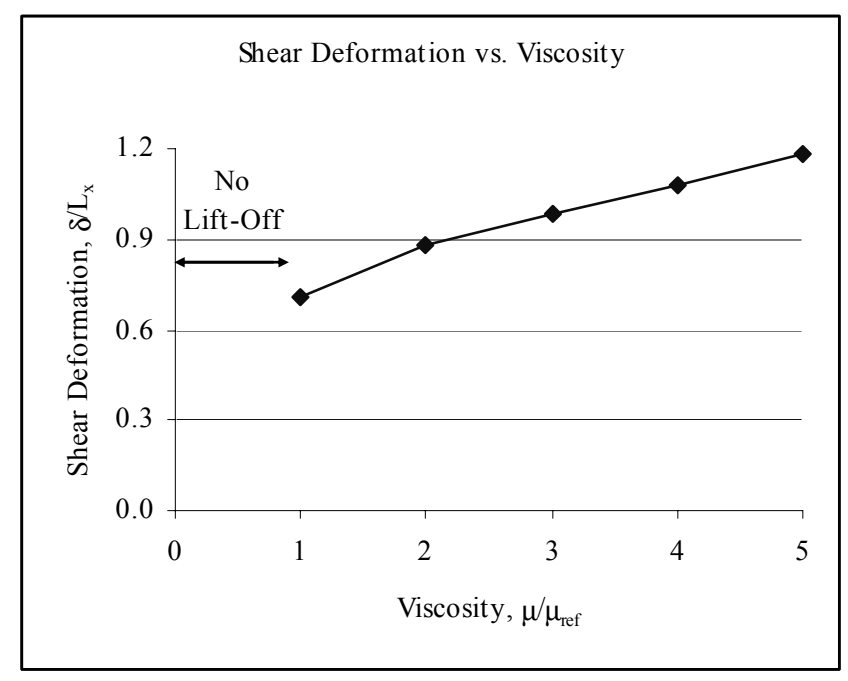

\subsection{Maximum Shear Deformation vs. Viscosity}

The effect of surface roughness, holding all other dimensional base parameters constant, is explored in the next set of results. Figure 5.31 shows a decrease in the dimensionless pumping rate as roughness heights increase. This result is misleading, as the pumping rate is non-dimensionalized with the surface roughness parameter $\sigma$. Since the dimensionless pumping rate includes a factor of $\sigma^{3}$, the expression for pumping rate shows that, in fact, the dimensional pumping rate is steadily rising with increasing roughness height (Figure 5.32). This effect is expected, as an increase in asperity heights means an increase in the asperities' ability to induce pumping. 


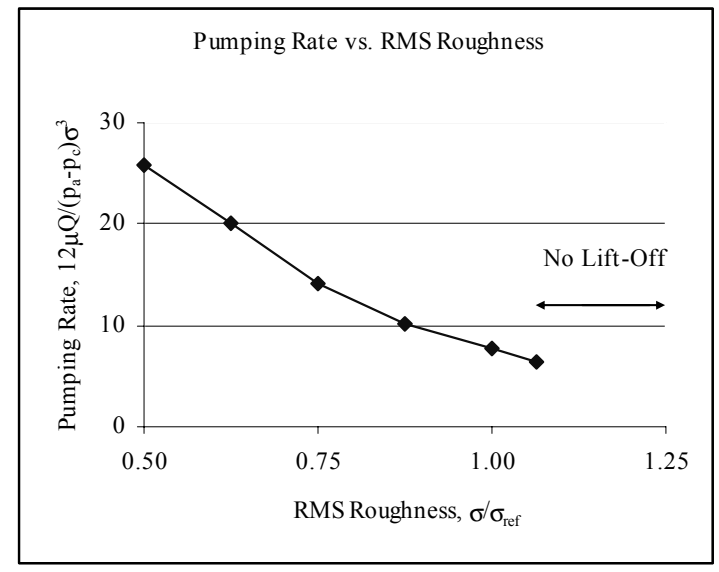

5.31 Pumping Rate vs. RMS Roughness

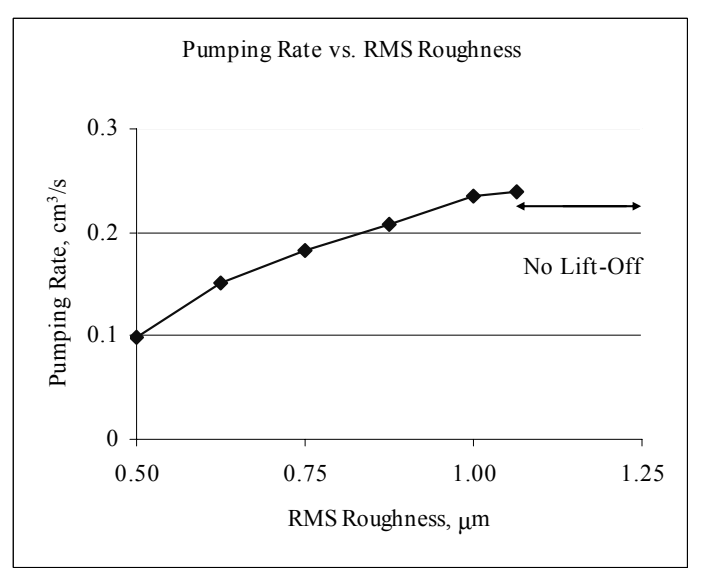

5.32 Dimensional Pumping Rate vs. RMS Roughness

Figure 5.33 shows a reduction in load support as asperity heights rise. This is because the value for dimensionless speed is reduced as roughness heights increase.

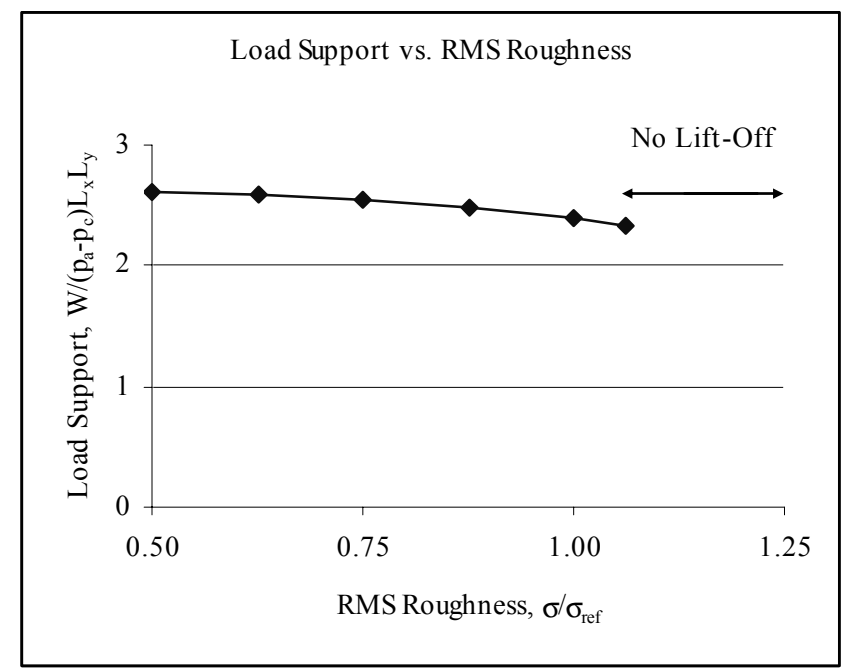

5.33 Load Support vs. RMS Roughnes 
The effect on power consumption shown in Figure 5.34 is not straight-forward, as it is also non-dimensionalized with the roughness parameter squared. In terms of the dimensional power loss (Figure 5.35), it is found that an increase in roughness heights has a minimal effect on power loss; a 19\% decrease in Watts vs. a 53\% increase in RMS roughness.

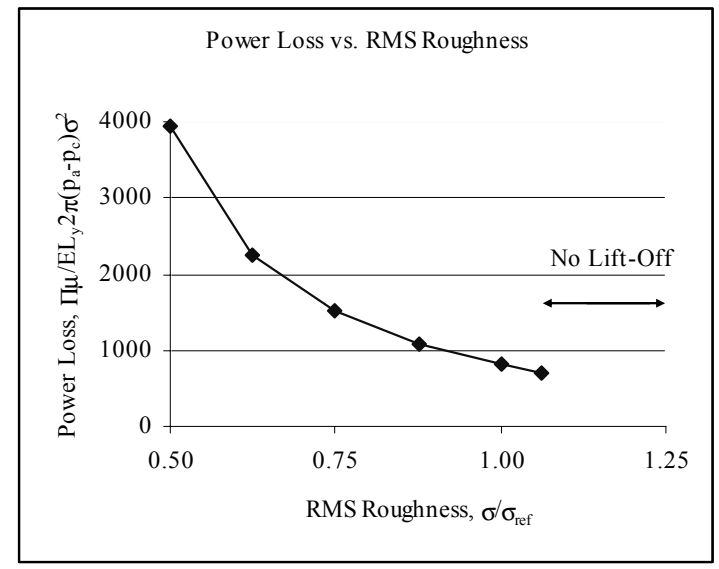

5.34 Power Loss vs. RMS Roughness

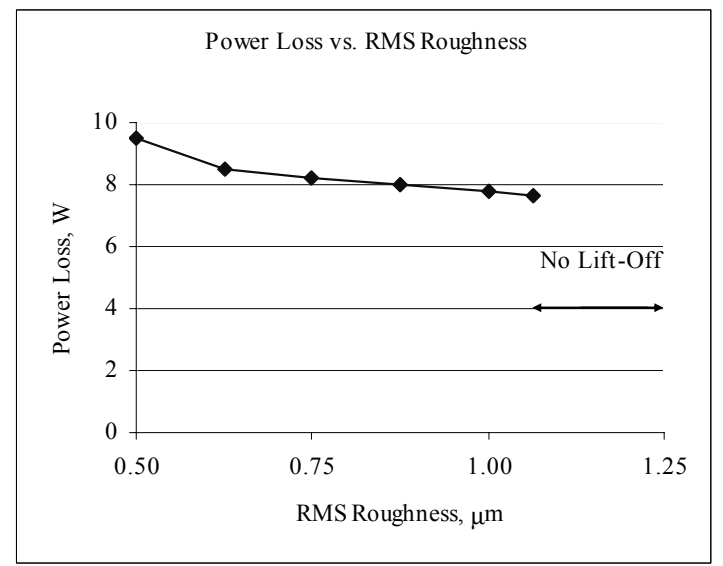

5.35 Dimensional Power Loss vs. RMS Roughness

Figure 5.36 shows a reduction in average film thickness as asperity heights increase. This is again because the value for dimensionless speed is reduced as roughness heights increase. 


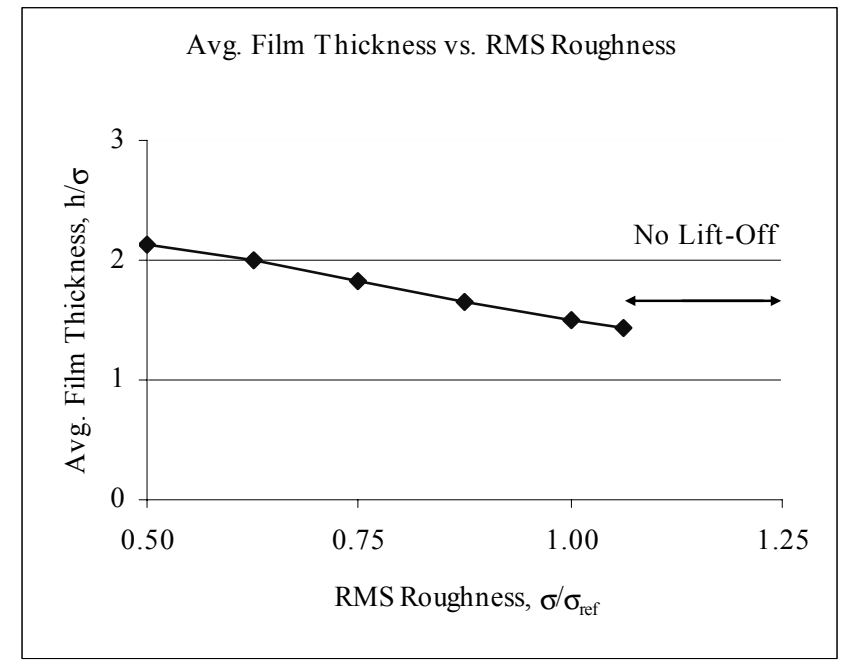

5.36 Average Film Thickness vs. RMS Roughness

A decrease in maximum shear deformation as roughness heights grow larger is shown in Figure 5.37. This effect is due to the lower dimensionless speed produced by a larger roughness parameter.

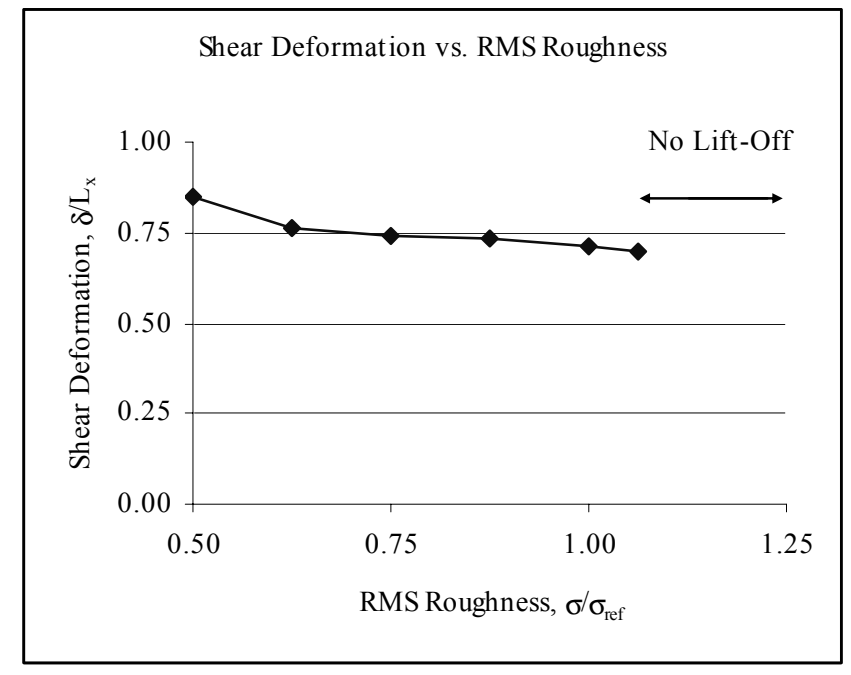

5.37 Maximum Shear Deformation vs. RMS Roughness 
The results in Figures 5.38 and 5.39 show the speeds with which the lip will lift off of the shaft at various viscosities and surface roughness heights, respectively. In Figure 5.38, the dimensionless lift-off speed is independent of the dimensionless viscosity. It is therefore clear that the dimensional speed required for lift-off is higher for lower viscosities (Figure 5.39), since the load support is proportional to the speed.

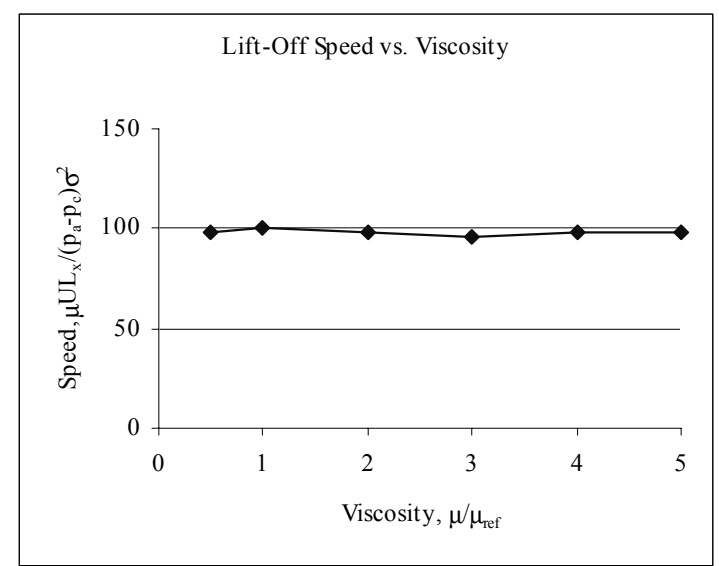

5.38 Lift-off Speed vs. Viscosity

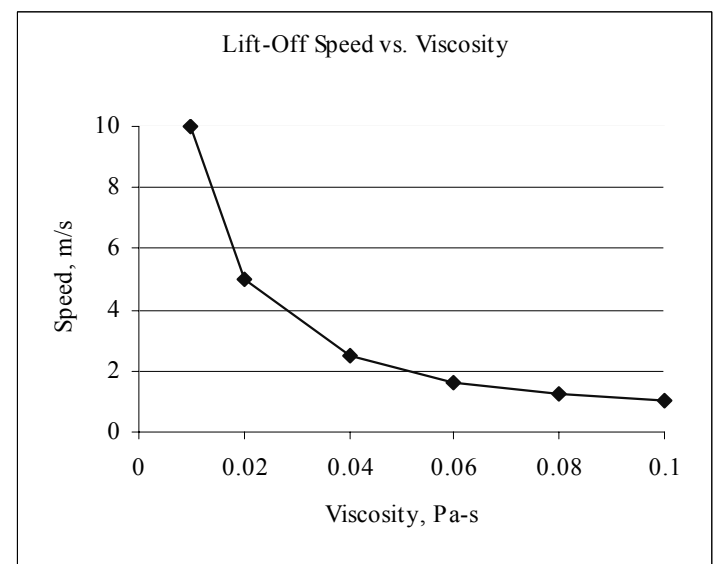

5.39 Dimensional Lift-off Speed vs. Viscosity

The graph of dimensionless lift-off speed for various roughness heights is another example in which the roughness parameter is part of the dimensionless term. Since the dimensionless velocity is inversely proportional to the square of $\sigma$, the expression for speed shows that, in fact, the dimensional speed is steadily rising with increasing roughness height (Figure 41). One explanation for this would be the effect of cavitation as roughness heights increase. 


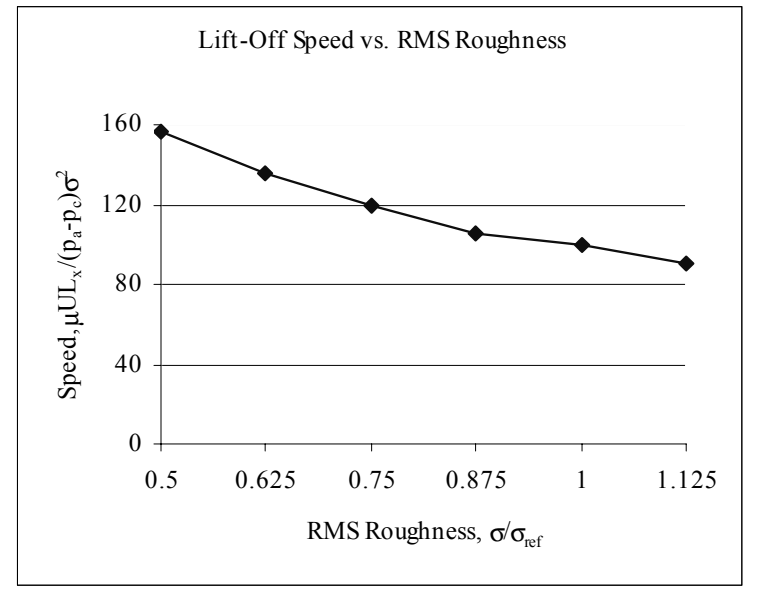

5.40 Lift-off Speed vs. RMS Roughness

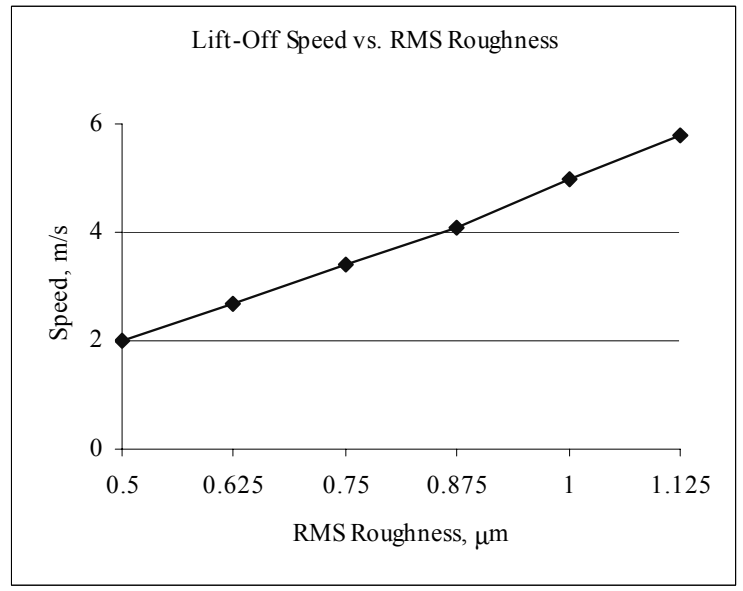

5.41 Dimensional Lift-off Speed vs. RMS Roughness

The following figures show the effects of asperity aspect ratio. In these cases, the undeformed asperity height, $\lambda_{\mathrm{y}}$ is kept at a constant value and the undeformed asperity width, $\lambda_{\mathrm{x}}$ is altered. Figure 5.42 shows an expected drop in pumping rate as $\gamma$ approaches lower values (asperities of a more circular shape), and an increase in pumping as the higher $\gamma$ values produce a more effective vane-like pumping mechanism. There is no liftoff for $\gamma<3.0$, and the largest pumping rate occurs when $\gamma$ is increased from 3.0 to 4.0. 


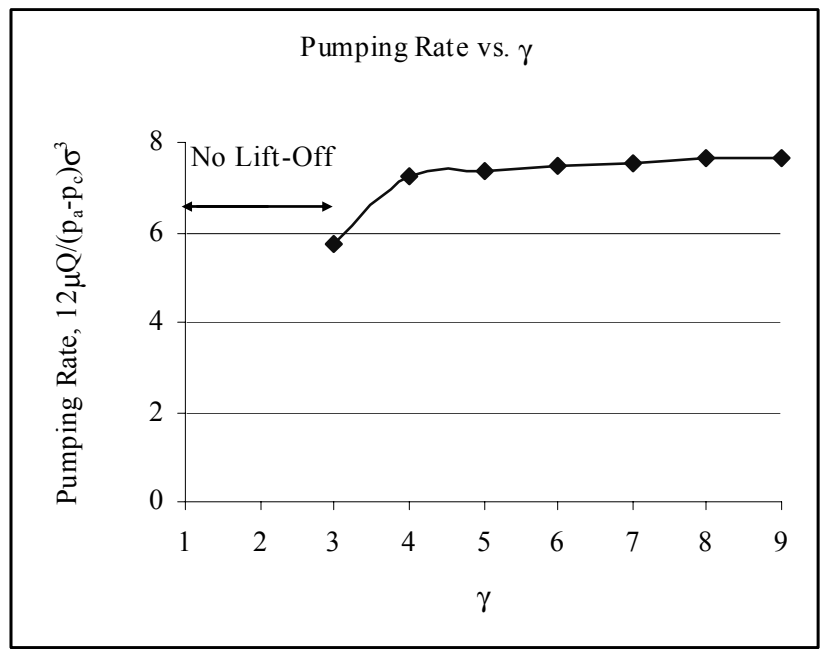

\subsection{Pumping Rate vs. Asperity Aspect Ratio}

There is a slight increase in load support and average film thickness (Figures 5.43 and 5.44) with larger $\gamma$ values and virtually no effect on power loss and shear deformation (Figures 5.45 and 5.46).

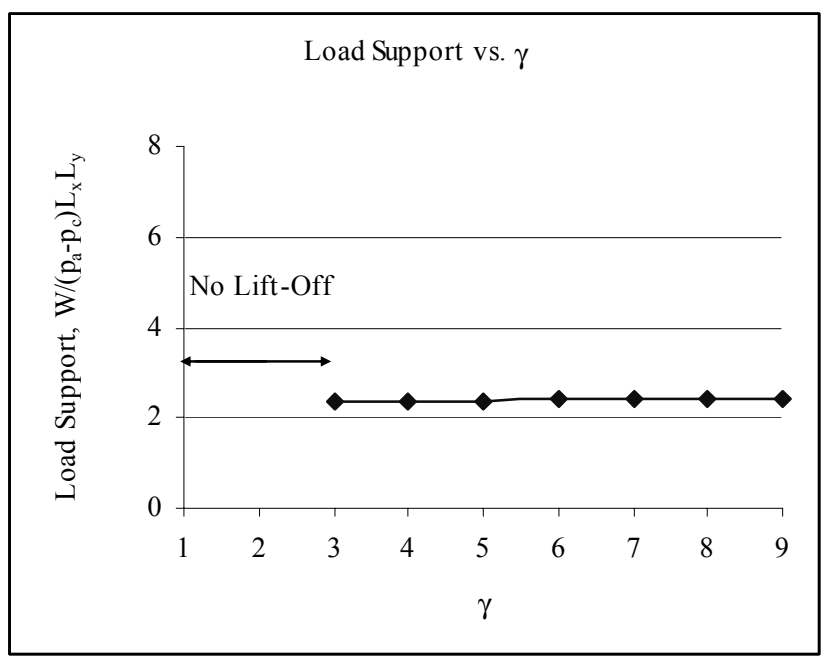

5.43 Load Support vs. Asperity Aspect Ratio 


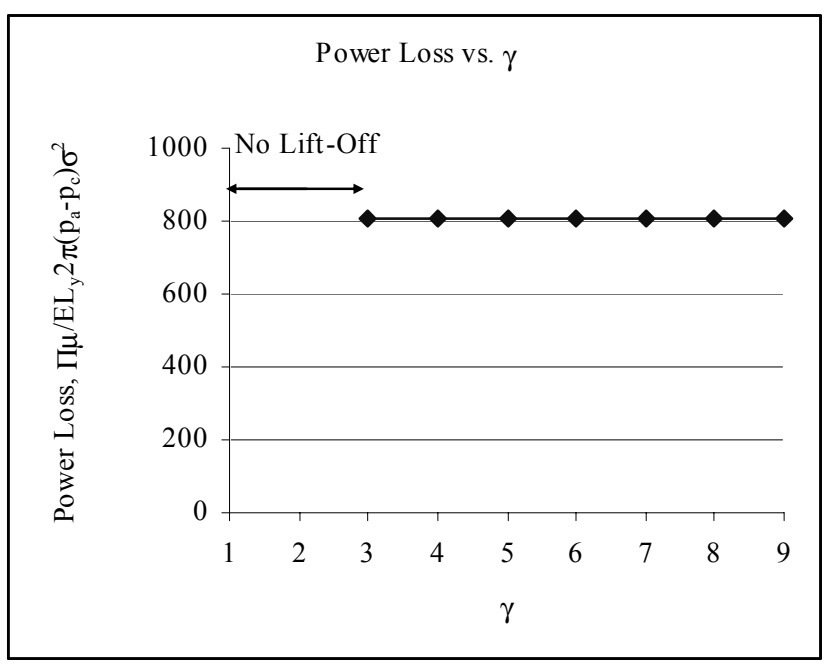

5.44 Power Loss vs. Asperity Aspect Ratio

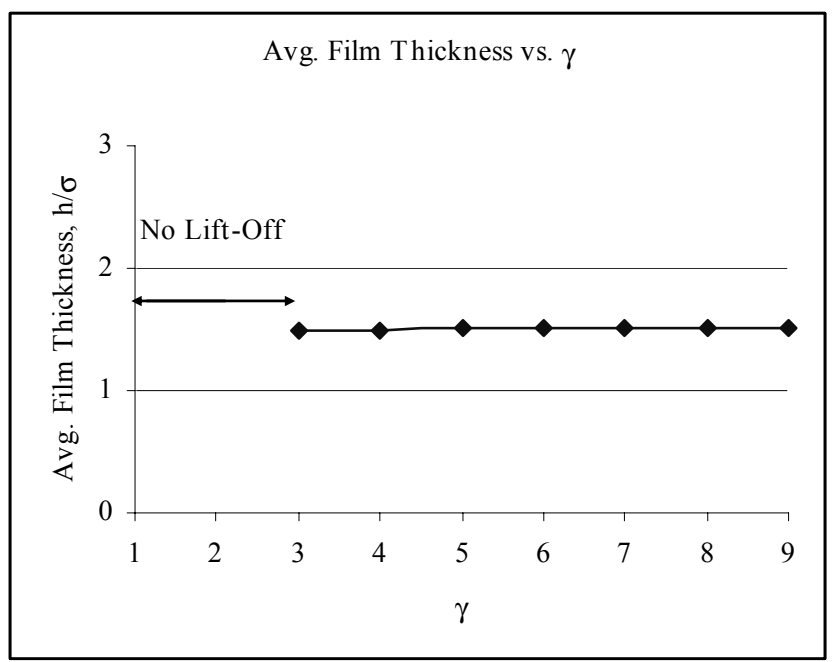

5.45 Avg. Film Thickness vs. Asperity Aspect Ratio 


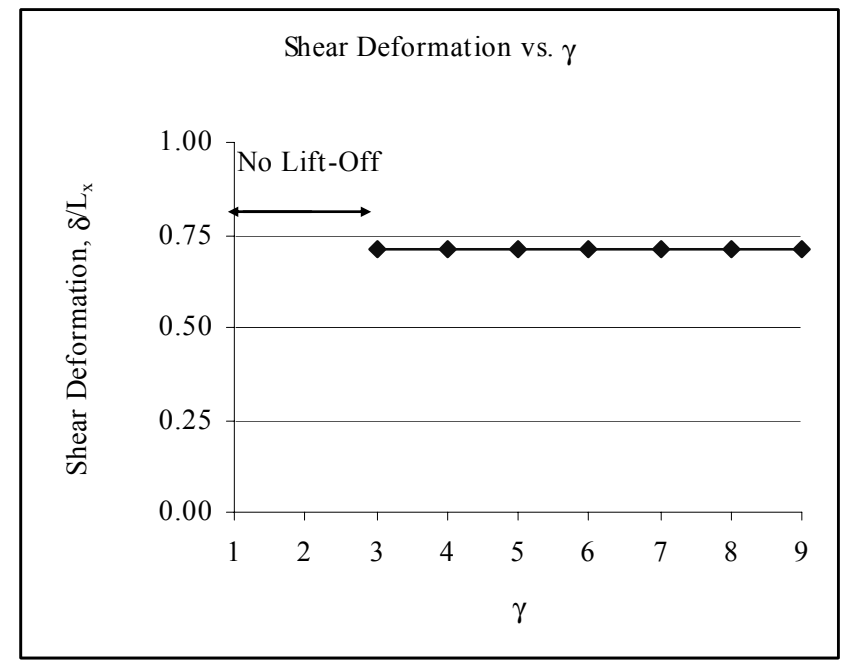

5.46 Maximum Shear Deformation vs. Asperity Aspect Ratio 


\section{CHAPTER VI}

\section{CONCLUSIONS}

A computational method has been developed to model the behaviors in a rotary

lip seal application. The method analyzes the fluid mechanics of the lubricating film and the elastic deformation of the lip by solving the Reynolds equation with flow factors. Due to its statistical approach, the method is more computationally efficient and includes a feature that addresses the varying asperity distortion due to the shear deformation of the lip surface. It was found that the method can predict such seal characteristics as the thickness of the lubricating film, reverse pumping rate, power dissipation and load carrying capacity and that the results are reasonable and agree qualitatively and quantitatively with previous studies. 


\section{REFERENCES}

Harp, S. R. and Salant, R. F., 2001, “An average flow model of rough surface lubrication with inter-asperity cavitation," ASME J. of Tribology, Vol. 123, pp. 134-143.

Horve, L. A., 1996, Shaft Seals forDynamic Applications (1st ed.). New York, Marcel Dekker.

Kammüller, M., 1986, Zur Abdichtwirkung von Radial-Wellendichtringen, Dr.-Ing. Thesis, Universität Stuttgart.

Lo, S-W, 1992, "On the effects of roughness orientation - a mapping and double scale analysis of flow factors," ASME J. of Tribology, Vol. 114, pp. 747-754.

Müller, H. K., 1987, "Concepts of sealing mechanism of rubber lip type rotary shaft seals," Proc. 11 th BHRA International Conference on Fluid Sealing, Cannes, France, pp. 698-709.

Patankar, S.V., 1980, Numerical Heat Transfer and Fluid Flow, New York, Hemisphere.

Patir, N. and Cheng, H. S., 1978, “An average flow model for determining effects of three-dimensional roughness on partial kydrodynamic lubrication," ASME Journal of Lubrication Technology, Vol. 100, pp. 12-17.

Patir, N. and Cheng, H. S., 1979, "Application of the average flow model to lubrication between rough sliding surfaces," ASME Journal of Lubrication Technology, Vol. 101, pp. 220-229.

Salant, R. F. and Flaherty, A. L., 1994, "Elastohydrodynamic analysis of reverse pumping in rotary lip seals with microundulations," ASME J. of Tribology, Vol. 116, pp. 56-62.

Salant, R. F. and Flaherty, A. L, 1995, "Elastohydrodynamic analysis of reverse pumping in rotary lip seals with microasperities," ASME J. of Tribology, Vol. 117, pp. 53-59.

Salant, R. F., and Rocke, A. H., 2004, "Hydrodynamic analysis of the flow in a rotary lip seal using flow factors," ASME J. of Tribology, Vol. 126, pp. 156 - 161.

Salant, R. F., 1996, "Elastohydrodynamic model of the rotary lip seal," ASME J. of Tribology, Vol. 118, pp. 292-296.

Salant, R. F., 1999, "Theory of lubrication of elastomeric rotary shaft seals," IMechE J. Engineering Tribology, Vol. 213, pp. 189-201. 
Shen, D., and Salant, R. F., 2003, "Elastohydrodynamic analysis of the effect of shaft surface finish on rotary lip seal behavior," STLE J. of Tribology, Vol. 46, pp. 391-396.

Shi, F. and Salant, R. F., 2000, “A mixed soft elastohydrodynamic lubrication model with interasperity cavitation and surface shear deformation," ASME J. of Tribology, Vol. 122, pp. 308-316.

Shi, F. and Salant, R. F., 2001, "Numerical study of a rotary lip seal with a quasirandom sealing surface," ASME J. of Tribology, Vol. 123, pp. 517-524.

Stakenborg, M. J. L., 1988, "On the sealing mechanism of radial lip seals," Tribology International, Vol. 21, pp. 335-340.

Thomas, G. B. Jr., 1958, Calculus and Analytic Geometry (2nd ed.). New Reading, Addison-Wesley.

Tzeng, S.T. and Saibel, E., 1967, "Surface roughness effect on slider bearing lubrication," ASLE Transactions, Vol. 10, pp. 334-338. 